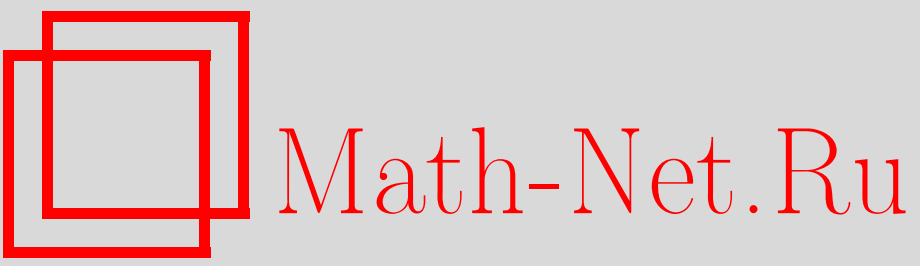

А. И. Булгаков, О. П. Беляева, А. А. Григоренко, К теории возмущенных включений и о ее приложениях, Матем. сб., 2005, том 196, номер 10, 21-78

DOI: https://doi.org/10.4213/sm1425

Использование Общероссийского математического портала Math-Net.Ru подразумевает, что вы прочитали и согласны с пользовательским соглашением

http://www.mathnet.ru/rus/agreement

Параметры загрузки:

IP: 54.198 .55 .26

26 апреля 2023 г., 18:16:08 


\section{К теории возмущенных включений и о ее приложениях}

В работе изучаются включения, правая часть которых представляет алгебраическую сумму значений компактнозначного оператора и отображения, являющегося произведением линейного интегрального оператора и многозначного оператора с вьпукльми по переключению образами. Для таких включений рассматриваются вопросы существования решений, доказаны принцип плотности и "бэнг-бэнг” принцип. Изучены свойства множеств решений включений с внутренними и внешними возмущениями. Получено необходимое и достаточное условие, когда пересечение замьканий множеств приближенных решений совпадает с замыканием множества решений исходного включения. Доказанные результаты применяются для изучения краевых задач функционально-дифференциальных включений.

Библиография: 49 названий.

В работе изучается включение, правая часть которого состоит из алгебраической суммы значений “хорошего" (имеюшего замкнутые образы) и “плохого" (не обладающего свойством замкнутости и выпуклости значений) многозначных отображений. Такие включения здесь назьваются возмущенными. Отметим, что все имеюшиеся в настоящее время методы исследования (теорема Какутани, принцип сжимающих отображений, метод непрерывных ветвей (см., например, [1], [2])) непосредственно применить для изучения вопросов существования решений таких возмушенных включений нельзя, поскольку оператор, порожденный правой частью, не обладает свойством замкнутости значений. В то же время к таким включениям сводятся многие задачи дифференциальных и интегральных включений, теорий аппроксимации, управления и игр. Поэтому построение основ теории возмушенных включений представляет не только теоретический, но и практический интерес.

Отметим, что возмущенные включения рассматривались в работах [3]-[6]. В этих статьях для случая, когда "хорошее" многозначное отображение имеет выпуклые замкнутые образы, рассмотрены вопросы существования решений возмушенных включений, а также топологические свойства множеств решений и квазирешений таких включений. В частности, в этих работах получены оценки близости решений к наперед заданной непрерывной функции, которые позволяют путем подбора функций определить приближенное решение возмушенного включения и дать оценку погрешности этого решения. Кроме того, доказано, что множество квазирешений возмушенного включения совпадает с множеством решений

Работа выполнена при финансовой поддержке Российского фонда фундаменталњњых исследований (гранты № № 01-01-00140, 04-01-00324), Министерства образования РФ (грант № E02-1.0-212). 
“овыпукленного” включения. На основе этого утверждения и полученных в [3]-[6] оценок доказан принцип плотности и “бэнг-бэнг” принцип. Доказательство этих свойств в [5] основывалось на теореме Майкла [7], с помощью которой доказывалось существование в некотором смысле "минимальной" непрерьвной ветви у "хорошего" многозначного отображения с выпуклыми образами. В настоящей работе не предполагается, что “хорошее” многозначное отображение имеет выпуклые образы. Поэтому применить теорему Майкла [7] для исследования такого возмущенного включения невозможно. Исследования в этом случае здесь осушествляются на основе теоремы 1 , доказанной в $\S 1$.

В $\S 2$ исследуются возмушенные включения с внешними и внутренними возмущениями. Здесь доказано, что "небольшими” внутренними и внешними возмушениями нельзя пренебрегать, поскольку они могут существенно изменить множество решений возмушенного включения. Таким образом, это доказьвает, что если правая часть включения не обладает свойством замкнутости значений, то может нарушаться принцип устойчивости множеств решений от "небольших" изменений правой части.

В $\S 3$ применяется теория, разработанная в $\S \S 1,2$ для изучения краевых задач функционально-дифференциальных включений.

\section{§0. Обозначения и некоторые определения}

Пусть $X$ - нормированное пространство с нормой $\|\cdot\|_{X}$. Через $B_{X}[x ; \varepsilon]$ обозначим открытый шар пространства $X$ с центром в точке $x \in X$ и радиусом $\varepsilon>0$; если $\varepsilon=0$, то $B_{X}[x ; 0] \equiv x$. Пусть $U \subset X$. Тогда $\bar{U}$ - замыкание множества $U$; со $U$ - выпуклая оболочка множества $U$; $\overline{\operatorname{co}} U \equiv \overline{\operatorname{co} U}$; ext $U$ - множество крайних точек множества $U ; \overline{\operatorname{ext}} U=\overline{\operatorname{ext} U} ;\|U\|_{X}=\sup _{u \in U}\|u\|_{X} ; \Omega(U)$ - множество всех непустых выпуклых подмножеств множества $U ; U^{\varepsilon} \equiv \overline{\bigcup_{u \in U} B[u ; \varepsilon]}$, если $\varepsilon>0$, и $U^{0} \equiv \bar{U} ; \rho_{X}[x ; U]-$ расстояние от точки $x \in X$ до множества $U$ в пространстве $X$; $h_{X}^{+}\left[U_{1} ; U\right] \equiv \sup _{x \in U_{1}} \rho_{X}[x ; U]-$ полуотклонение по Хаусдорфу множества $U_{1} \subset X$ от множества $U$ в пространстве $X ; h_{X}\left[U_{1} ; U\right]=\max \left\{h^{+}\left[U_{1} ; U\right], h^{+}\left[U ; U_{1}\right]\right\}$ - pacстояние по Хаусдорфу между множествами $U_{1}$ и $U$ в пространстве $X ; \operatorname{comp}[X]$ $\left(\operatorname{comp}\left[X^{*}\right]\right)$ - множество всех непустых компактов пространства $X$ (множество всех непустых ограниченных замкнутых в пространстве $X$ предкомпактных в слабой топологии пространства $X$ подмножеств).

Пусть $\mathbb{R}^{n}-n$-мерное пространство вектор-столбцов с нормой $|\cdot|$. Через $C^{n}[a, b]$ $\left(D^{n}[a, b]\right)$ обозначим пространство непрерывных (абсолютно непрерывных) функций $x:[a, b] \rightarrow \mathbb{R}^{n}$ с нормой

$$
\|x\|_{C^{n}[a, b]}=\max \{|x(t)|: t \in[a, b]\} \quad\left(\|x\|_{D^{n}[a, b]}=|x(a)|+\int_{a}^{b}|\dot{x}(s)| d s\right) .
$$

Пусть $\mathscr{U} \subset[a, b]$ - измеримое множество, $\mu(\mathscr{U})>0\left(\mu-\right.$ мера Лебега), через $L^{n}(\mathscr{U})$ обозначим пространство суммируемых функций $x: \mathscr{U} \rightarrow \mathbb{R}^{n}$ с нормой

$$
\|x\|_{L^{n}(\mathscr{U})}=\int_{\mathscr{U}}|x(s)| d s .
$$


Пусть $\Phi \subset L^{n}[a, b]$. Будем говорить, что множество $\Phi$ выпукло по переключению (разложимо), если для любых $x, y \in \Phi$ и любого измеримого множества $\mathscr{U} \subset[a, b]$ выполняется включение $\chi(\mathscr{U}) x+\chi([a, b] \backslash \mathscr{U}) y \in \Phi$, где $\chi(\cdot)$ - характеристическая функция соответствующего множества. Обозначим через $\Pi\left[L^{n}[a, b]\right]$ множество всех непустых ограниченных замкнутых выпуклых по переключению подмножеств пространства $L^{n}[a, b]$. Через $\Omega\left(\Pi\left[L^{n}[a, b]\right]\right)$ обозначим множество всех непустых выпуклых ограниченных замкнутых выпуклых по переключению подмножеств пространства $L^{n}[a, b]$.

Пусть $F:[a, b] \rightarrow \operatorname{comp}\left[\mathbb{R}^{n}\right]$ - измеримое отображение. Введем обозначение $S(F)=\left\{y \in L^{n}[a, b]: y(t) \in F(t)\right.$ при п.в. $\left.t \in[a, b]\right\}$.

Через $C_{+}^{1}[a, b]\left(L_{+}^{1}[a, b]\right)$ обозначим конус неотрицательных функций пространства $C^{1}[a, b]\left(L^{1}[a, b]\right)$.

Измеримость однозначных функций везде понимается здесь по Лебегу, измеримость многозначных функций понимается в смысле [8].

Если $X=\mathbb{R}^{n}$, то в этом случае для сокращения записи индекс $\mathbb{R}^{n}$ в обозначениях расстояний опускаем.

Ниже приведем основные характеристические свойства выпуклых по переключению множеств, которые будут использоваться в дальнейшем.

Лемма 1. Пусть $\Phi \in \Pi\left[L^{n}[a, b]\right]$. Тогда существует такая функиия $и \in$ $L^{1}[a, b]$, что для любой функиии $\varphi \in \Phi$ и для почти всех $t \in[a, b]$ выполняется оченка $|\varphi(t)| \leqslant u(t)$.

ДокАЗАТЕЛЬСтво. Пусть последовательность функций $\varphi_{i} \in \Phi, i=1,2, \ldots$, такова, что

$$
\lim _{i \rightarrow \infty}\left\|\varphi_{i}\right\|_{L^{n}[a, b]}=\|\Phi\|_{L^{n}[a, b]} .
$$

Покажем, что найдется такая последовательность функций $\widetilde{\varphi}_{i} \in \Phi, i=1,2, \ldots$, для которой вьполняется равенство (1) и при почти всех $t \in[a, b]$ имеют место соотношения

$$
\left|\widetilde{\varphi}_{1}(t)\right| \leqslant\left|\widetilde{\varphi}_{2}(t)\right| \leqslant\left|\widetilde{\varphi}_{3}(t)\right| \leqslant \cdots \leqslant\left|\widetilde{\varphi}_{i}(t)\right| \leqslant\left|\widetilde{\varphi}_{i+1}(t)\right| \leqslant \cdots .
$$

Действительно, пусть $\widetilde{\varphi}_{1}=\varphi_{1}$ и $\widetilde{\varphi}_{i+1}=\chi\left(\mathscr{U}_{i}\right) \widetilde{\varphi}_{i}+\chi\left([a, b] \backslash \mathscr{U}_{i}\right) \varphi_{i+1}, i=1,2, \ldots$, где $\mathscr{U}_{i}=\left\{t \in[a, b]:\left|\widetilde{\varphi}_{i}(t)\right| \geqslant\left|\varphi_{i+1}(t)\right|\right\}$. Так как $\Phi \in \Pi\left[L^{n}[a, b]\right]$, то последовательность $\widetilde{\varphi}_{i} \in \Phi, i=1,2, \ldots$, обладает следующими свойствами: при почти всех $t \in[a, b]$ выполняются неравенства (2); для любого $i=1,2, \ldots$ справедлива оценка $\left\|\widetilde{\varphi}_{i}\right\|_{L^{n}[a, b]} \geqslant\left\|\varphi_{i}\right\|_{L^{n}[a, b]}$. Следовательно, из последнего свойства, а также равенства (1) вытекает, что последовательность $\widetilde{\varphi}_{i}, i=1,2, \ldots$, удовлетворяет равенству (1). Далее, определим измеримую функцию $u:[a, b] \rightarrow[0, \infty)$ равенстBOM

$$
u(t)=\lim _{i \rightarrow \infty}\left|\widetilde{\varphi}_{i}(t)\right| .
$$

Так как множество $\Phi$ ограничено в $L^{n}[a, b]$, то согласно лемме Фату (см. [9]) функция $u \in L^{1}[a, b]$. Кроме того, из определения функции $u$ и равенства (1) для любого измеримого множества $\mathscr{U} \subset[a, b]$ выполняется соотношение

$$
\int_{\mathscr{U}} u(t) d t=\|\Psi\|_{L^{n}(\mathscr{U})}
$$


Теперь покажем, что функция $u$, заданная соотношением (3), удовлетворяет утверждению леммы. Действительно, предположим противное. Тогда найдутся функция $\varphi \in \Phi$ и измеримое множество $\mathscr{U}_{1} \subset[a, b]\left(\mu\left(\mathscr{U}_{1}\right)>0\right)$ такие, что для любого $t \in \mathscr{U}_{1}$ выполняется оценка $|\varphi(t)|>u(t)$. Отсюда вытекает, что

$$
\int_{\mathscr{U}_{1}}|\varphi(t)| d t>\int_{\mathscr{U}_{1}} u(t) d t
$$

но это противоречит равенству (4). Лемма доказана.

ЛЕМма 2. Пусть $\Phi \in \Pi\left[L^{n}[a, b]\right]$, u nусть $\varphi_{i} \in \Phi, i=1,2, \ldots,-$ noследовательность, плотная в $\Phi$. Далее, пусть измеримое отображение $F:[a, b] \rightarrow \operatorname{comp}\left[\mathbb{R}^{n}\right]$ определено равенством

$$
F(t)=\overline{\left\{\varphi_{i}(t), i=1,2, \ldots\right\}} .
$$

Тогда справедливо равенство $S(F)=\Phi$.

ДокаЗАтельство. Так как $\varphi_{i} \in S(F)$, а последовательность $\varphi_{i}, i=1,2, \ldots$, плотна в $\Phi$, то в силу замкнутости множества $\Phi$ получаем вложение $\Phi \subset S(F)$. Докажем теперь, что $S(F) \subset \Phi$. Пусть $x \in S(F)$. Для каждых $k, i=1,2, \ldots$ обозначим измеримые множества

$$
E_{i}^{k}=\left\{t \in[a, b]:\left|x(t)-\varphi_{i}(t)\right| \leqslant \frac{1}{k}\right\} .
$$

Для $i=1$ пусть $\widetilde{E}_{1}^{k}=E_{1}^{k}$, а для $i=2,3, \ldots-\widetilde{E}_{i}^{k}=E_{1}^{k} \backslash \bigcup_{j=1}^{i-1} E_{j}^{k}$. Тогда $\widetilde{E}_{i}^{k} \cap \widetilde{E}_{j}^{k}=\varnothing$, если $i \neq j$. Согласно определению отображения $F:[a, b] \rightarrow$ $\operatorname{comp}\left[\mathbb{R}^{n}\right]$ для любого $k=1,2, \ldots$ имеет место соотношение

$$
\mu\left(\bigcup_{i=1}^{\infty} \widetilde{E}_{i}^{k}\right)=b-a
$$

Определим последовательность измеримых функций $x_{k}:[a, b] \rightarrow \mathbb{R}^{n}, k=1,2, \ldots$, равенствами

$$
x_{k}(t)= \begin{cases}\varphi_{i}(t), & t \in \widetilde{E}_{i}^{k}, i=1,2, \ldots, k ; \\ \varphi_{1}(t), & t \in[a, b] \backslash \bigcup_{i=1}^{k} \widetilde{E}_{i}^{k} .\end{cases}
$$

Из выпуклости по переключению множества $\Phi$ вытекает, что $x_{k} \in \Phi$ для любого $k=1,2, \ldots$ Кроме того, согласно лемме 1 и определению множеств $\widetilde{E}_{i}^{k}$ для функции $x_{k}, k=1,2, \ldots$, получаем оценку

$$
\left\|x-x_{k}\right\|_{L^{n}[a, b]} \leqslant \frac{b-a}{k}+2 \int_{[a, b] \backslash \bigcup_{i=1}^{k} \widetilde{E}_{i}^{k}} u(t) d t,
$$

где функция $u$ удовлетворяет утверждению леммы 1 . Из равенства (5) и соотношения (6) следует, что $x_{k} \rightarrow x$ в пространстве $L^{n}[a, b]$ при $k \rightarrow \infty$. Так как множество $\Phi$ замкнуто, то $x \in \Phi$ и, следовательно, $S(F) \subset \Phi$. Таким образом, $S(F)=\Phi$. Лемма доказана. 
ЛЕмма 3. Пусть измеримые отображсения

$$
F_{i}:[a, b] \rightarrow \operatorname{comp}\left[\mathbb{R}^{n}\right], \quad i=1,2, \ldots
$$

ограничень суммируемыми функциями. Имеем $S\left(F_{1}\right) \subset S\left(F_{2}\right)$ тогда и только тогда, когда $F_{1}(t) \subset F_{2}(t)$ при почти всех $t \in[a, b]$.

ДокАЗАТЕЛЬСтво. Прежде всего, если $F_{1}(t) \subset F_{2}(t)$ при почти всех $t \in[a, b]$, то, очевидно, $S\left(F_{1}\right) \subset S\left(F_{2}\right)$.

Пусть теперь $S\left(F_{1}\right) \subset S\left(F_{2}\right)$, и пусть $\varphi_{i} \in L^{n}[a, b], i=1,2, \ldots,-$ счетное плотное в $S\left(F_{1}\right)$ множество, аппроксимируюшее отображение $F_{1}:[a, b] \rightarrow \operatorname{comp}\left[\mathbb{R}^{n}\right]$ (см. [8]). Тогда $\varphi_{i} \in S\left(F_{2}\right)$ для любого $i=1,2, \ldots$, и поэтому при почти всех $t \in[a, b]$ справедливо вложение $\left\{\varphi_{i}(t): i=1,2, \ldots\right\} \subset F_{2}(t)$. Так как последовательность $\varphi_{i}, i=1,2, \ldots$, аппроксимирует отображение $F_{1}:[a, b] \rightarrow \operatorname{comp}\left[\mathbb{R}^{n}\right]$, то из последнего соотношения следует, что $F_{1}(t) \subset F_{2}(t)$ при почти всех $t \in[a, b]$. Лемма доказана.

СлЕДСТВИЕ 1. Пусть $\Phi \in \Pi\left[L^{n}[a, b]\right]$, и пусть $F_{i}:[a, b] \rightarrow \operatorname{comp}\left[\mathbb{R}^{n}\right], i=1,2$, - такие измеримые отображения, что выполняются соотношения $\Phi=$ $S\left(F_{1}\right)=S\left(F_{2}\right)$. Тогда при почти всех $t \in[a, b]$ выполняется равенство $F_{1}(t)=F_{2}(t)$.

ЗАмечание 1 . Таким образом, если $\Phi \in \Pi\left[L^{n}[a, b]\right]$, то измеримое отображение $F:[a, b] \rightarrow \operatorname{comp}\left[\mathbb{R}^{n}\right]$, удовлетворяюшее условию $S(F)=\Phi$, однозначно определяет множество $\Phi$.

\section{§1. Некоторые результаты возмущенного}

\section{включения с компактнозначным отображением}

Рассмотрим в пространстве $C^{n}[a, b]$ включение

$$
x \in \Psi(x)+V \Phi(x),
$$

где сумма понимается как алгебраическая сумма множеств,

$$
\Psi: C^{n}[a, b] \rightarrow \operatorname{comp}\left[C^{n}[a, b]\right], \quad \Phi: C^{n}[a, b] \rightarrow \Pi\left[L^{n}[a, b]\right]
$$

- многозначные отображения, линейный непрерывный интегральньй оператор $V: L^{n}[a, b] \rightarrow C^{n}[a, b]$ определен равенством

$$
(V z)(t)=\int_{a}^{b} V(t, s) z(s) d s, \quad t \in[a, b] .
$$

Включение (7) назовем возмущеннылм включением.

Под решением включения (7) будем понимать элемент $x \in C^{n}[a, b]$, удовлетворяющий (7). Таким образом, непрерывная функция $x:[a, b] \rightarrow \mathbb{R}^{n}$ является решением включения (7) тогда и только тогда, когда найдутся такие элементы $v \in \Psi(x)$ и $z \in \Phi(x)$, что справедливо равенство $x=v+V z$. 
Пусть $q_{0} \in C^{n}[a, b], r_{0} \in \Psi\left(q_{0}\right)$ и $w_{0} \in L^{n}[a, b]$. Представим функцию $q_{0}$ в виде

$$
q_{0}=r_{0}+V w_{0}+e,
$$

где $e=q_{0}-r_{0}-V w_{0}$. Предположим, что функция $k \in L^{1}[a, b]$ для каждого измеримого $\mathscr{U} \subset[a, b]$ удовлетворяет неравенству

$$
\rho_{L^{n}(\mathscr{U})}\left[w_{0} ; \Phi\left(q_{0}\right)\right] \leqslant \int_{\mathscr{U}} k(s) d s
$$

а непрерывная функция $\nu:[a, b] \rightarrow[0, \infty)$ определена соотношением

$$
\nu(t)=\int_{a}^{b}|V(t, s)| k(s) d s+|e(t)|
$$

где $|V(t, s)|$ - согласованная с пространством $\mathbb{R}^{n}$ норма $n \times n$ матрицы $V(t, s)$ в представлении $(8), e \in C^{n}[a, b]$ - функция из правой части равенства (9).

ОПРЕДЕЛЕНИЕ. Будем говорить, что отображения $V: L^{n}[a, b] \rightarrow C^{n}[a, b]$, $\Psi: C^{n}[a, b] \rightarrow \operatorname{comp}\left[C^{n}[a, b]\right], \Phi: C^{n}[a, b] \rightarrow \Pi\left[L^{n}[a, b]\right]$ обладают свойством $\mathrm{A}$, если найдутся непрерывные изотонные операторы $\Gamma: C_{+}^{1}[a, b] \rightarrow L_{+}^{1}[a, b]$ и $P$ : $C_{+}^{1}[a, b] \rightarrow \mathbb{R}^{1}$, удовлетворяющие следующим условиям: для любых $x, y \in C^{n}[a, b]$ и любого измеримого множества $\mathscr{U} \subset[a, b]$ выполняются неравенства

$$
\begin{aligned}
h_{L^{n}(\mathscr{U})}[\Phi(x) ; \Phi(y)] & \leqslant\|\Gamma(Z(x-y))\|_{L^{1}(\mathscr{U})}, \\
h_{C^{n}[a, b]}[\Psi(x) ; \Psi(y)] & \leqslant P(Z(x-y)) ;
\end{aligned}
$$

для функции $\nu \in C_{+}^{1}[a, b]$, определенной соотношением (11), сходится в пространстве $C^{1}[a, b]$ ряд

$$
\sum_{i=0}^{\infty} \mathscr{A}^{i} \nu, \quad \mathscr{A}^{0} \nu=\nu, \quad \mathscr{A}^{i} \nu=\mathscr{A}\left(\mathscr{A}^{i-1} \nu\right), \quad i=1,2, \ldots,
$$

где непрерывный оператор $\mathscr{A}: C_{+}^{1}[a, b] \rightarrow C_{+}^{1}[a, b]$ определен равенством

$$
(\mathscr{A} z)(t)=\int_{a}^{b}|V(t, s)|(\Gamma z)(s) d s+P(z),
$$

а отображение $Z: C^{n}[a, b] \rightarrow C_{+}^{1}[a, b]$ определено соотношением

$$
(Z x)(t)=|x(t)| \text {. }
$$

Пусть $\xi(\nu)$ - сумма ряда $(14)$, т.е.

$$
\xi(\nu)=\sum_{i=0}^{\infty} \mathscr{A}^{i} \nu
$$


ОПРЕДЕЛЕНИЕ. Будем говорить, что отображения

$V: L^{n}[a, b] \rightarrow C^{n}[a, b], \quad \Psi: C^{n}[a, b] \rightarrow \operatorname{comp}\left[C^{n}[a, b]\right], \quad \Phi: C^{n}[a, b] \rightarrow \Pi\left[L^{n}[a, b]\right]$

обладают свойством $\mathrm{A}^{*}$, если найдутся непрерывные изотонные операторы $\Gamma$ : $C_{+}^{1}[a, b] \rightarrow L_{+}^{1}[a, b]$ и $P: C_{+}^{1}[a, b] \rightarrow \mathbb{R}^{1}$, удовлетворяюшие неравенствам (12), (13), а также соотношениям $\Gamma(0)=0, P(0)=0$, и, кроме того, для любой функции $\widetilde{\nu} \in$ $C_{+}^{1}[a, b]$ из некоторой окрестности нуля ряд (14) сходится в пространстве $C^{1}[a, b]$ и его сумма непрерьвна в нуле.

Teopema 1. Пусть $q_{0} \in C^{n}[a, b], r_{0} \in \Psi\left(q_{0}\right), w_{0} \in L^{n}[a, b]$, и пусть функция q

$V: L^{n}[a, b] \rightarrow C^{n}[a, b], \quad \Psi: C^{n}[a, b] \rightarrow \operatorname{comp}\left[C^{n}[a, b]\right], \quad \Phi: C^{n}[a, b] \rightarrow \Pi\left[L^{n}[a, b]\right]$

обладают свойством А. Тогда найдется такое решение $x(x=v+V z$, $v \in \Psi(x), z \in \Phi(x))$ включения (7), для которого выполняются следующие оченки: при любом $t \in[a, b]$

$$
\begin{aligned}
\left|x(t)-q_{0}(t)\right| & \leqslant \xi(\nu)(t), \\
\left\|v-r_{0}\right\|_{C^{n}[a, b]} & \leqslant P(\xi(\nu)) ;
\end{aligned}
$$

при почти всех $t \in[a, b]$

$$
\left|z(t)-w_{0}(t)\right| \leqslant k(t)+(\Gamma \xi(\nu))(t),
$$

где $\nu, \xi(\nu), P, \Gamma, k$ удовлетворяют соотношениям (11), (17), (13), (12), (10) соответственно.

ДокАЗАТЕЛЬСтво. Пусть функция $q_{0} \in C^{n}[a, b]$ представима в виде (9), и пусть функция $w_{1} \in \Phi\left(q_{0}\right)$ для любого измеримого $\mathscr{U} \subset[a, b]$ удовлетворяет равенству

$$
\left\|w_{1}-w_{0}\right\|_{L^{n}(\mathscr{U})}=\rho_{L^{n}(\mathscr{U})}\left[w_{0} ; \Phi\left(q_{0}\right)\right] .
$$

Тогда в силу неравенства (10) при почти всех $t \in[a, b]$ справедлива оценка

$$
\left|w_{1}(t)-w_{0}(t)\right| \leqslant k(t) .
$$

Далее, пусть $q_{1}=r_{1}+V w_{1}$, где $r_{1} \equiv r_{0}$. Тогда согласно (21) для любого $t \in[a, b]$ получаем соотношение

$$
\left|q_{1}(t)-q_{0}(t)\right| \leqslant \int_{a}^{b}|V(t, s)| k(s) d s+|e(t)|=\left(\mathscr{A}^{0} \nu\right)(t) .
$$

Предположим, что функция $r_{2} \in \Psi\left(q_{1}\right)$ удовлетворяет равенству

$$
\left\|r_{2}-r_{1}\right\|_{C^{n}[a, b]}=\rho_{C^{n}[a, b]}\left[r_{1} ; \Psi\left(q_{1}\right)\right] .
$$


Тогда в силу (13) имеет место неравенство

$$
\left\|r_{2}-r_{1}\right\|_{C^{n}[a, b]} \leqslant P\left(\mathscr{A}^{0} \nu\right)
$$

Пусть для функции $w_{2} \in \Phi\left(q_{1}\right)$ и любого измеримого множества $\mathscr{U} \subset[a, b]$ справедливо соотношение

$$
\left\|w_{2}-w_{1}\right\|_{L^{n}(\mathscr{U})}=\rho_{L^{n}(\mathscr{U})}\left[w_{1} ; \Phi\left(q_{1}\right)\right]
$$

Из определения функции $w_{2}$ и неравенств $(12),(22)$ для любого измеримого $\mathscr{U} \subset$ $[a, b]$ вытекает оценка

$$
\left\|w_{2}-w_{1}\right\|_{L^{n}(\mathscr{U})} \leqslant\left\|\Gamma \mathscr{A}^{0} \nu\right\|_{L^{1}(\mathscr{U})} .
$$

Пусть $q_{2}=r_{2}+V w_{2}$. Тогда при всех $t \in[a, b]$ справедливо соотношение

$$
\left|q_{2}(t)-q_{1}(t)\right| \leqslant(\mathscr{A} \nu)(t)
$$

Продолжая этот процесс дальше, получаем последовательности $\left\{q_{i}\right\},\left\{r_{i}\right\}$ и $\left\{w_{i}\right\}$ такие, что для любого $i=1,2, \ldots$ справедливо равенство

$$
q_{i}=r_{i}+V w_{i}
$$

где $r_{i} \in \Psi\left(q_{i-1}\right), w_{i} \in \Phi\left(q_{i-1}\right)$, причем имеют место следующие соотношения:

$$
\begin{aligned}
\left|q_{i}(t)-q_{i-1}(t)\right| & \leqslant\left(\mathscr{A}^{i-1} \nu\right)(t), \\
\left\|r_{i+1}-r_{i}\right\|_{C^{n}[a, b]} & \leqslant P\left(\mathscr{A}^{i-1} \nu\right)
\end{aligned}
$$

и при почти всех $t \in[a, b]$

$$
\left|w_{i+1}(t)-w_{i}(t)\right| \leqslant\left(\Gamma \mathscr{A}^{i-1} \nu\right)(t)
$$

Из оценки (24) вытекает, что для любых $j=0,1,2, \ldots, i=1,2, \ldots$ и при любом $t \in[a, b]$ выполняются соотношения

$$
\left|q_{j+i}(t)-q_{j}(t)\right| \leqslant \sum_{m=j}^{\infty}\left(\mathscr{A}^{m} \nu\right)(t)
$$

Из сходимости ряда (14) вытекает сходимость последовательности $\left\{q_{i}\right\}$. Пусть $x=\lim _{i \rightarrow \infty} q_{i}$. Докажем, что $x$ и есть искомое решение. Прежде всего, из неравенства (27) вытекает оценка (18).

Далее, согласно (25) для любых $i, j=1,2, \ldots$ справедливо соотношение

$$
\left\|r_{j+i}-r_{j}\right\|_{C^{n}[a, b]} \leqslant P\left(\sum_{m=j-1}^{\infty} \mathscr{A}^{m} \nu\right)
$$


Из неравенства (28) вытекает, что последовательность $\left\{r_{i}\right\}$ сходится. Пусть $v=$ $\lim _{i \rightarrow \infty} r_{i}$. Покажем, что $v$ удовлетворяет неравенству (19). Действительно, принимая в соотношении (28) $j=1$ и переходя к пределу при $i \rightarrow \infty$ при этом учитывая, что $r_{1} \equiv r_{0}$, получаем оценку (19).

Наконец, рассмотрим последовательность $\left\{w_{i}\right\}$. Из оценки (26) для любых $j, i=1,2, \ldots$ и при почти всех $t \in[a, b]$ имеет место соотношение

$$
\left|w_{j+i}(t)-w_{j}(t)\right| \leqslant \Gamma\left(\sum_{m=j-1}^{\infty} \mathscr{A}^{m} \nu\right)(t) .
$$

Из (29) вытекает, что последовательность $\left\{w_{i}\right\}$ сходится. Полагая $z=\lim _{i \rightarrow \infty} w_{i}$, из оценок (21), (29) получаем, что $z$ удовлетворяет неравенству (20).

Далее, переходя в неравенстве (23) к пределу при $i \rightarrow \infty$, получаем равенство

$$
x=v+V z,
$$

причем из непрерывности по Хаусдорфу отображений $\Psi: C^{n}[a, b] \rightarrow \operatorname{comp}\left[C^{n}[a, b]\right]$, $\Phi: C^{n}[a, b] \rightarrow \Pi\left[L^{n}[a, b]\right]$ вытекают включения $v \in \Psi(x)$ и $z \in \Phi(x)$, т.е. $x-$ решение включения (7). Теорема доказана.

ЗАмечАниЕ 2. Отметим, что теорема 1 дополняет результат работы [5], в которой аналогичные оценки получены в случае выпуклозначности отображения $\Psi: C^{n}[a, b] \rightarrow \operatorname{comp}\left[C^{n}[a, b]\right]$. При этом в [5] доказательство этих оценок основывалось на теореме Майкла [7], с помошью которой доказывалось существование в некотором смысле "минимальной" непрерывной ветви $g: C^{n}[a, b] \rightarrow C^{n}[a, b]$ отображения $\Psi: C^{n}[a, b] \rightarrow \operatorname{comp}\left[C^{n}[a, b]\right]$, а также с помощью результата работы [10]. Предложенную в работе [5] схему для доказательства теоремы 1 применить невозможно, поскольку теорема 1 не предполагает, что отображение $\Psi$ : $C^{n}[a, b] \rightarrow \operatorname{comp}\left[C^{n}[a, b]\right]$ выпуклозначно.

ЗАмечАнИЕ 3 . Отметим, что теорема 1 не является непосредственным следствием принципа сжимаюших отображений (см. [8]), поскольку оператор, порожденный правой частью включения (7), не является замкнутозначньм.

ЗАмечание 4. Отметим, что теорема 1 дает несколько больше, чем просто условия сушествования решения включения (7). Она дает способ нахождения приближенного решения путем подбора функции $q_{0} \in C^{n}[a, b]$. При этом функция $\xi(\nu)$, зависящая от функций $q_{0}, r_{0} \in C^{n}[a, b]$ и $w_{0} \in L^{n}[a, b]$, дает оценку погрешности приближенного решения (функции $q_{0}$ ) включения (7).

ОПреДЕЛЕНИЕ. Будем говорить, что непрерывный оператор $\mathscr{A}: C_{+}^{1}[a, b] \rightarrow$ $C_{+}^{1}[a, b]$, определенный равенством (15), аддитивен на әлементе $\nu \in C_{+}^{1}[a, b]$, заданном соотношением (11), если для любого $m=1,2, \ldots$ имеет место равенство

$$
\mathscr{A}\left(\sum_{i=0}^{m} \mathscr{A}^{i} \nu\right)=\sum_{i=1}^{m+1} \mathscr{A}^{i} \nu .
$$


ЗАмЕчАниЕ 5. Отметим, что если непрерывный оператор $\mathscr{A}: C_{+}^{1}[a, b] \rightarrow C_{+}^{1}[a, b]$, определенный равенством (15), аддитивен на элементе $\nu \in C_{+}^{1}[a, b]$, заданном соотношением (11), то сумма ряда (14) является решением уравнения

$$
\xi(\nu)=\mathscr{A}(\xi(\nu))+\nu .
$$

Пусть функция $p:[a, b] \rightarrow \mathbb{R}^{1}$ измерима по Лебегу и при почти всех $t \in[a, b]$ удовлетворяет оценке $p(t) \leqslant t$, а функция $w: \mathbb{R}^{1} \backslash[a, b] \rightarrow \mathbb{R}^{n}$ ограничена и измерима по Борелю. Определим непрерывный оператор $\Theta: C^{n}[a, b] \rightarrow L^{n}[a, b]$ равенством

$$
(\Theta x)(t)= \begin{cases}x[p(t)], & p(t) \in[a, b] \\ w[p(t)], & p(t) \notin[a, b]\end{cases}
$$

В качестве приложения теоремы 1 рассмотрим задачу Коши для дифференциального включения с запаздыванием

$$
\dot{x}(t) \in F(t,(\Theta x)(t)), \quad t \in[a, b], \quad x(a)=x_{0},
$$

где отображение $F:[a, b] \times \mathbb{R}^{n} \rightarrow \operatorname{comp}\left[\mathbb{R}^{n}\right]$ обладает следующими свойствами: для всех $x \in \mathbb{R}^{n}$ отображение $F(\cdot, x)$ измеримо (см. [8]); сушествует такая функция $\beta \in L_{+}^{1}[a, b]$, что при почти всех $t \in[a, b]$ и всех $x, y \in \mathbb{R}^{n}$ выполняется неравенство

$$
h[F(t, x) ; F(t, y)] \leqslant \beta(t)|x-y|
$$

сушествует такая функция $\gamma \in L_{+}^{1}[a, b]$, что при почти всех $t \in[a, b]$ имеет место оценка $\|F(t, 0)\| \leqslant \gamma(t)$.

Под решением задачи (31) будем понимать абсолютно непрерьвную функцию $x:[a, b] \rightarrow \mathbb{R}^{n}$, при почти всех $t \in[a, b]$ удовлетворяющую включению $(31)$ и равенству $x(a)=x_{0}$.

Напомним, что многозначный оператор Немыцкого $\mathscr{N}: L^{n}[a, b] \rightarrow \Pi\left[L^{n}[a, b]\right]$, порожденньй отображением $F:[a, b] \times \mathbb{R}^{n} \rightarrow \operatorname{comp}\left[\mathbb{R}^{n}\right]$, определяется равенством

$$
\mathscr{N}(x)=\left\{y \in L^{n}[a, b]: y(t) \in F(t, x(t)) \text { при п.в. } t \in[a, b]\right\} .
$$

Очевидно, что задача (31) эквивалентна интегральному включению

$$
x \in x_{0}+\Lambda \mathscr{N}(\Theta x),
$$

где оператор $\Lambda: L^{n}[a, b] \rightarrow D^{n}[a, b]$ определен равенством

$$
(\Lambda z)(t)=\int_{a}^{t} z(s) d s
$$

Этот оператор будем называть оператором интегрирования. Включение (33) является частным случаем (7). При этом отображение $\widetilde{\Psi}: C^{n}[a, b] \rightarrow \operatorname{comp}\left[C^{n}[a, b]\right]$ в данном случае определяется равенством $\widetilde{\Psi}(x)=x_{0}$. 
Пусть функция $q_{0}:[a, b] \rightarrow \mathbb{R}^{n}$ абсолютно непрерывна. В этом случае равенство (9) принимает вид

$$
q_{0}=x_{0}+\Lambda \dot{q}_{0}+e
$$

где $e=q_{0}(a)-x_{0}, \dot{q}_{0} \in L^{n}[a, b]-$ производная функции $q_{0}$. Далее, пусть функция $\widetilde{k} \in L_{+}^{1}[a, b]$ при почти всех $t \in[a, b]$ удовлетворяет неравенству

$$
\rho\left[\dot{q}_{0}(t) ; F\left(t,\left(\Theta q_{0}\right)(t)\right)\right] \leqslant \widetilde{k}(t) .
$$

Отметим, что функция $\widetilde{k}$ удовлетворяет неравенству $(10)$, в котором $\Phi-$ произведение оператора Немыцкого $\mathcal{N}: L^{n}[a, b] \rightarrow \Pi\left[L^{n}[a, b]\right]$, порожденного отображением $F:[a, b] \times \mathbb{R}^{n} \rightarrow \operatorname{comp}\left[\mathbb{R}^{n}\right]$, и отображения $\Theta: C^{n}[a, b] \rightarrow L^{n}[a, b]$. Для рассматриваемого случая непрерывная функция $\nu:[a, b] \rightarrow[0, \infty)$ имеет вид

$$
\nu(t)=\int_{a}^{t} \widetilde{k}(s) d s+\left|q_{0}(a)-x_{0}\right|
$$

Покажем, что отображения

$$
\begin{aligned}
\Lambda: L^{n}[a, b] \rightarrow & C^{n}[a, b], \quad \widetilde{\Psi}: C^{n}[a, b] \rightarrow \operatorname{comp}\left[C^{n}[a, b]\right], \\
& \mathcal{N} \Theta: C^{n}[a, b] \rightarrow \Pi\left[L^{n}[a, b]\right]
\end{aligned}
$$

обладают свойством А. Действительно, для этих отображений непрерывные изотонные операторы $\Gamma: C_{+}^{1}[a, b] \rightarrow L_{+}^{1}[a, b]$ и $P: C_{+}^{1}[a, b] \rightarrow \mathbb{R}^{1}$ определяются равенствами

$$
(\Gamma z)(t)=\beta(t) \max _{s \in[a, t]} z(s), \quad P(z)=0
$$

где функция $\beta \in L_{+}^{1}[a, b]$ удовлетворяет неравенству (32), а непрерьвньй оператор $\mathscr{A}: C_{+}^{1}[a, b] \rightarrow C_{+}^{1}[a, b]$ задан соотношением

$$
(\mathscr{A} z)(t)=\int_{a}^{t} \beta(s) \max _{\tau \in[a, s]} z(\tau) d s
$$

Так как для функции $\nu$, заданной соотношением $(34)$, для любого $t \in[a, b]$ справедливо равенство

$$
(\mathscr{A} \nu)(t)=\int_{a}^{t} \beta(s) \max _{\tau \in[a, s]} \nu(\tau) d s=\int_{a}^{t} \beta(s) \nu(s) d s
$$

то непрерывный оператор $\mathscr{A}: C_{+}^{1}[a, b] \rightarrow C_{+}^{1}[a, b]$, определенный равенством $(35)$, аддитивен на элементе $\nu \in C_{+}^{1}[a, b]$.

Поэтому в силу замечания 5 сумма ряда (14) в данном случае представляет собой решение уравнения

$$
\xi(\nu)(t)=\int_{a}^{t} \beta(s) \xi(\nu)(s) d s+\nu(t)
$$


где функция $\nu \in C_{+}^{1}[a, b]$ удовлетворяет равенству (34). Решение уравнения (36) имеет вид

$$
\xi(\nu)(t)=\left|x_{0}-q_{0}(a)\right| e^{\varphi(t)}+\int_{a}^{t} e^{\varphi(t)-\varphi(s)} \widetilde{k}(s) d s,
$$

где функция $\varphi \in C_{+}^{1}[a, b]$ задается равенством

$$
\varphi(t)=\int_{a}^{t} \beta(s) d s .
$$

Таким образом, из теоремы 1 вытекает оценка А. $\Phi$. Филиппова (см. [11], [12]) для дифференциального включения с запаздыванием.

Ниже рассматриваются свойства множеств решений и квазирешений возмушенного включения (7) в пространстве непрерывных функций.

ОпРеДЕлЕнИЕ. Аналогично [5] будем говорить, что функция $x \in C^{n}[a, b]$ sвляется квазирешением включения (7), если найдутся такой элемент $v \in \Psi(x)$ и такая последовательность

$$
z_{i} \in \Phi(x), \quad i=1,2, \ldots,
$$

что последовательность $x_{i}=v+V z_{i} \rightarrow x$ в $C^{n}[a, b]$ при $i \rightarrow \infty$. Через $\mathscr{H}$ обозначим множество всех квазирешений включения (7).

Пусть $U \subset C^{n}[a, b]$. Далее, будем считать, что если квазирешение включения $(7) x \in U$, то найдутся такой элемент $v \in \Psi(x)$ и такая последовательность $z_{i} \in L^{n}[a, b], i=1,2, \ldots$, удовлетворяюшая включению (37), что для любого $i=1,2, \ldots$ выполняется включение $x_{i}=v+V z_{i} \in U$ и $x_{i} \rightarrow x$ в $C^{n}[a, b]$ при $i \rightarrow \infty$.

Отметим, что для многих задач (например, периодических, многоточечных краевых задач) это свойство квазирешений включения (7), можно доказать непосредственно (см. [6]), используя теорему 2 (см. ниже). Кроме того, если оператор, определенный правой частью включения (7), переводит вьпуклое замкнутое множество $U \subset C^{n}[a, b]$ в себя, то согласно теореме 2 (см. ниже) квазирешения включения (7) обладают вышеупомянутым свойством на множестве $U$.

Рассмотрим в пространстве $C^{n}[a, b]$ включение

$$
x \in \Psi(x)+V \overline{\overline{c o}}(\Phi(x)) .
$$

Включение (38) по аналогии с [5] будем называть “овыпукленным. возмущенным включением или просто овыпукленныцм включением.

Пусть $H_{\text {со }}$ - множество решений включения (38). Справедливо следуюшее утверждение для квазирешений включения (7).

Tеорема 2. Пусть линейный непрерывный оператор $V: L^{n}[a, b] \rightarrow C^{n}[a, b]$, определенный равенством (8), переводит каждое слабо компактное в $L^{n}[a, b]$ множество в предкомпактное множество пространства $C^{n}[a, b]$. Тогда справедливо равенство $\mathscr{H}=H_{\text {со }}$. 
Доказательство. Сначала докажем, что $H_{\text {со }} \subset \mathscr{H}$. Пусть $x \in H_{\text {со }}$. Тогда найдутся такие функции $v \in \Psi(x)$ и $w \in \overline{\mathrm{co}}(\Phi(x))$, что выполняется равенство $x=v+V w$. Согласно [13] для функции $w$ найдется такая последовательность $w_{i} \in \Phi(x), i=1,2, \ldots$, что $w_{i} \rightarrow w$ слабо в $L^{n}[a, b]$ при $i \rightarrow \infty$. Это означает, что при любом $t \in[a, b]$

$$
y_{i}(t)=v(t)+\left(V w_{i}\right)(t) \rightarrow x(t)=v(t)+(V w)(t)
$$

Так как последовательность $\left\{V w_{i}\right\}$ компактна в $C^{n}[a, b]$, то можно считать, что $y_{i} \rightarrow x$ в $C^{n}[a, b]$ при $i \rightarrow \infty$. Следовательно, $H_{\text {со }} \subset \mathscr{H}$.

Докажем теперь вложение $\mathscr{H} \subset H_{\text {со }}$. Пусть $x \in \mathscr{H}$. Тогда найдутся $v \in \Psi(x)$ и последовательность $w_{i} \in \Phi(x)$ такие, что $y_{i}=v+V w_{i} \rightarrow x$ в пространстве $C^{n}[a, b]$ при $i \rightarrow \infty$. Поскольку последовательность $\left\{w_{i}\right\}$ слабо компактна в $L^{n}[a, b]$, то, не уменьшая общности, можно считать, что $w_{i} \rightarrow w$ слабо в $L^{n}[a, b]$ при $i \rightarrow \infty$ для некоторого $w \in L^{n}[a, b]$. Так как $w_{i} \in \overline{\mathrm{co}}(\Phi(x))$, то $w \in \overline{\mathrm{co}}(\Phi(x))$ (cм. [8]) и справедливо равенство $x=v+V w$, т.е. $x \in H_{\text {со }}$. Следовательно, $\mathscr{H} \subset H_{\text {со }}$. Теорема доказана.

ЗАмЕчАнИЕ 6 . Отметим, что теорема 2 справедлива без какой-либо непрерывности отображений $\Psi: C^{n}[a, b] \rightarrow \operatorname{comp}\left[C^{n}[a, b]\right]$ и $\Phi: C^{n}[a, b] \rightarrow \Pi\left[L^{n}[a, b]\right]$.

ЗАмЕчАниЕ 7. Понятие квазитраектории для дифференциальных включений дано Важевским (Т. Wazewski, см. [12], [14], [15]). Приведенное выше определение квазирешения возмушенного включения с точки зрения дифференциальных включений несколько отличается от определения квазитраектории по Важевскому наличием условия (37) (это условие для дифференциальных включений означает, что производные функций $x_{i}, i=1,2, \ldots$, принадлежат значению $\mathscr{N}(x)$ оператора Немшцкого $\mathscr{N}: C^{n}[a, b] \rightarrow \Pi\left[L^{n}[a, b]\right]$, порожденного отображением $F:[a, b] \times \mathbb{R}^{n} \rightarrow \operatorname{comp}\left[\mathbb{R}^{n}\right]$ - правой частью дифференциального включения). Отметим, что сформулированное выше определение квазирешения более удобно для приложений. Кроме того, наличие включения (37) позволяет доказать основное свойство квазирешений (теорема 2) при более общих условиях даже для дифференциальных включений (не предполагая непрерьвности отображения $F:[a, b] \times \mathbb{R}^{n} \rightarrow$ $\operatorname{comp}\left[\mathbb{R}^{n}\right]$ по второму аргументу, для этого достаточно потребовать суперпозиционную измеримость отображения $F(\cdot, \cdot)$ (см. [5], [16]). Отметим также, что впервые свойства квазирешений (квазитраекторий) были использованы А.Ф. Филипповьм (см. [12]) для доказательства принципа плотности задачи Коши для дифференциального включения с невыпуклой правой частью.

Пусть многозначное отображение $\Delta:[a, b] \times C^{n}[a, b] \rightarrow \operatorname{comp}\left[\mathbb{R}^{n}\right]$ обладает свойством: при каждом фиксированном $x \in C^{n}[a, b]$ отображение $\Delta(\cdot, x)$ измеримо и удовлетворяет равенству

$$
\Phi(x)=\left\{y \in L^{n}[a, b]: y(t) \in \Delta(t, x) \text { при п.в. } t \in[a, b]\right\} .
$$

Такое отображение сушествует (см. лемму 2). 
Заметим, что для любого $x \in C^{n}[a, b]$ многозначное отображение $\Delta(\cdot, x):[a, b] \rightarrow$ comp $\left[\mathbb{R}^{n}\right]$ ограничено суммируемой функцией (см. лемму 1$)$.

Отметим, что если отображение $\Phi: C^{n}[a, b] \rightarrow \Pi\left[L^{n}[a, b]\right]$ есть многозначный оператор Немыцкого, порожденный функцией $F:[a, b] \times \mathbb{R}^{n} \rightarrow \operatorname{comp}\left[\mathbb{R}^{n}\right]$, то для оператора Немыцкого отображение $\Delta_{1}:[a, b] \times C^{n}[a, b] \rightarrow \operatorname{comp}\left[\mathbb{R}^{n}\right]$ определяется равенством

$$
\Delta_{1}(t, x)=F(t, x(t))
$$

Поэтому в этом случае можно отождествить $\Delta_{1}(\cdot, \cdot)$ с $F(\cdot, \cdot)$. В связи с этим в обшем случае естественно назвать отображение $\Delta:[a, b] \times C^{n}[a, b] \rightarrow \operatorname{comp}\left[\mathbb{R}^{n}\right]$ отображением, порождающим оператор $\Phi: C^{n}[a, b] \rightarrow \Pi\left[L^{n}[a, b]\right]$.

ОпределЕниЕ. Рассмотрим многозначное отображение $\widetilde{\Delta}:[a, b] \times C^{n}[a, b] \rightarrow$ $\operatorname{comp}\left[\mathbb{R}^{n}\right]$. Отображение $\widetilde{\Delta}(\cdot, \cdot)$ назовем интегрально непрерывныцм в точке $x \in C^{n}[a, b]$, если для любой последовательности $x_{i} \in C^{n}[a, b], i=1,2, \ldots$, сходяшейся к $x$ в пространстве $C^{n}[a, b]$ при $i \rightarrow \infty$, вьполняется равенство

$$
\lim _{i \rightarrow \infty} \int_{a}^{b} h\left[\widetilde{\Delta}\left(t, x_{i}\right) ; \widetilde{\Delta}(t, x)\right] d t=0 .
$$

Отображение $\widetilde{\Delta}:[a, b] \times C^{n}[a, b] \rightarrow \operatorname{comp}\left[\mathbb{R}^{n}\right]$ назовем интегрально непрерывныц.м на пространстве $C^{n}[a, b]$, если оно интегрально непрерывно в каждой точке $x \in C^{n}[a, b]$.

Лемма 4. Пусть множества $\Phi_{i} \in \Pi\left[L^{n}[a, b]\right], i=1,2$, и измеримые отображения $\Delta_{i}:[a, b] \rightarrow \operatorname{comp}\left[\mathbb{R}^{n}\right], i=1,2$, связаны между собой соотношениями $\Phi_{i}=S\left(\Delta_{i}(\cdot)\right), i=1,2$. Тогда для любого измеримого множества $\mathscr{U} \subset[a, b]$ выполняются неравенства

$$
h_{L^{n}(\mathscr{U})}\left[\Phi_{1} ; \Phi_{2}\right] \leqslant \int_{\mathscr{U}} h\left[\Delta_{1}(t) ; \Delta_{2}(t)\right] d t \leqslant 2 h_{L^{n}(\mathscr{U})}\left[\Phi_{1} ; \Phi_{2}\right] .
$$

ДокАЗАТЕЛЬСТво. Пусть $\mathscr{U} \subset[a, b]$ - измеримое множество. Обозначим

$$
\widetilde{\mathscr{U}}=\left\{t \in \mathscr{U}: h^{+}\left[\Delta_{1}(t) ; \Delta_{2}(t)\right] \geqslant h^{+}\left[\Delta_{2}(t) ; \Delta_{1}(t)\right]\right\} .
$$

Множество $\widetilde{\mathscr{U}} \subset \mathscr{U}$ измеримо. Далее, так как имеет место равенство

$$
\int_{\mathscr{U}} h\left[\Delta_{1}(t) ; \Delta_{2}(t)\right] d t=\int_{\widetilde{\mathscr{U}}} h^{+}\left[\Delta_{1}(t) ; \Delta_{2}(t)\right] d t+\int_{\mathscr{U} \backslash \widetilde{\mathscr{U}}} h^{+}\left[\Delta_{2}(t) ; \Delta_{1}(t)\right] d t,
$$

то получаем оценку

$$
\int_{\mathscr{U}} h\left[\Delta_{1}(t) ; \Delta_{2}(t)\right] d t \leqslant h_{L^{n}(\widetilde{\mathscr{U}})}^{+}\left[\Phi_{1} ; \Phi_{2}\right]+h_{L^{n}(\mathscr{U} \backslash \widetilde{\mathscr{U}})}^{+}\left[\Phi_{2} ; \Phi_{1}\right] .
$$

Из последнего соотношения вытекает оценка (40). Лемма доказана.

Из оценки (40) следует 
Teорема 3. Отображсние $\Phi: C^{n}[a, b] \rightarrow \Pi\left[L^{n}[a, b]\right]$ непрерывно по Хаусдорфу в точке в том и только том случае, когда отображсение $\Delta:[a, b] \times$ $C^{n}[a, b] \rightarrow \operatorname{comp}\left[\mathbb{R}^{n}\right]$, порождающее отображение $\Phi: C^{n}[a, b] \rightarrow \Pi\left[L^{n}[a, b]\right]$, интегрально непрерывно в этой точке.

Определим отображение со $\Delta:[a, b] \times C^{n}[a, b] \rightarrow \operatorname{comp}\left[\mathbb{R}^{n}\right]$ равенством

$$
(\operatorname{co} \Delta)(t, x)=\operatorname{co}(\Delta(t, x))
$$

ЗАмечаниЕ 8. Поскольку для любого $x \in C^{n}[a, b]$ имеет место равенство $\overline{\mathrm{co}}(\Phi(x))=S(\operatorname{co}(\Delta(\cdot, x)))\left(\right.$ см. [17]), то согласно [17] для любых $x, y \in C^{n}[a, b]$ и для любого измеримого $\mathscr{U} \subset[a, b]$ получим соотношение

$$
h_{L^{n}(\mathscr{U})}[\overline{\mathrm{co}}(\Phi(x)) ; \overline{\mathrm{co}}(\Phi(y))] \leqslant \int_{\mathscr{U}} h[\operatorname{co}(\Delta(t, x)) ; \operatorname{co}(\Delta(t, y))] d t .
$$

Так как при любых $t \in[a, b]$ и $x \in C^{n}[a, b] \Delta(t, x) \in \operatorname{comp}\left[\mathbb{R}^{n}\right]$, то из [18] для любого измеримого $\mathscr{U} \subset[a, b]$ вытекает оценка

$$
\int_{\mathscr{U}} h[\operatorname{co}(\Delta(t, x)) ; \operatorname{co}(\Delta(t, y))] d t \leqslant \int_{\mathscr{U}} h[\Delta(t, x) ; \Delta(t, y)] d t,
$$

где отображение $\Delta:[a, b] \times C^{n}[a, b] \rightarrow \operatorname{comp}\left[\mathbb{R}^{n}\right]$ порождает отображение $\Phi$ : $C^{n}[a, b] \rightarrow \Pi\left[L^{n}[a, b]\right]$. Поэтому из леммы 4 и неравенств $(41),(42)$ для любого измеримого множества $\mathscr{U} \subset[a, b]$ получаем соотношение

$$
h_{L^{n}(\mathscr{U})}[\overline{\mathrm{CO}}(\Phi(x)) ; \overline{\mathrm{CO}}(\Phi(y))] \leqslant 2 h_{L^{n}(\mathscr{U})}[\Phi(x) ; \Phi(y)] .
$$

Следовательно, из непрерывности по Хаусдорфу многозначного отображения $\Phi$ : $C^{n}[a, b] \rightarrow \Pi\left[L^{n}[a, b]\right]$ следует непрерывность по Хаусдорфу многозначного отображения со $\Phi: C^{n}[a, b] \rightarrow \Omega\left(\Pi\left[L^{n}[a, b]\right]\right)$, заданного равенством

$$
(\operatorname{co} \Phi)(x)=\overline{\operatorname{co}}(\Phi(x)) .
$$

ОПРЕДЕЛЕНИЕ. Будем говорить, что отображение $\Phi: C^{n}[a, b] \rightarrow \Pi\left[L^{n}[a, b]\right]$ ocлабленно замкнуто в точке $x \in C^{n}[a, b]$ (см. [19]), если для любой последовательности $x_{i}, i=1,2, \ldots$, сходящейся в пространстве $C^{n}[a, b]$ при $i \rightarrow \infty$ к $x$, любой последовательности $y_{i} \in \Phi\left(x_{i}\right), i=1,2, \ldots$, сходящейся слабо в $L^{n}[a, b]$ при $i \rightarrow \infty$ к $y$, выполняется включение $y \in \Phi(x)$. Если отображение $\Phi$ ослабленно замкнуто в каждой точке пространства $C^{n}[a, b]$, то будем говорить, что отображение $\Phi$ ослабленно замкнуто на $C^{n}[a, b]$.

ЛЕмма 5. Если отображение со $\Phi: C^{n}[a, b] \rightarrow \Omega\left(\Pi\left[L^{n}[a, b]\right]\right)$ полунепрерьвно сверху по Хаусдорфу на $C^{n}[a, b]$, то отображсение со $\Phi$ ослабленно замкнуто на $C^{n}[a, b]$. 
ДоКАЗАТЕЛЬСтво. Пусть последовательность $x_{i}, i=1,2, \ldots$, сходится к $x$ в пространстве $C^{n}[a, b]$ при $i \rightarrow \infty$, а последовательность $y_{i} \in(\operatorname{co} \Phi)\left(x_{i}\right)$ слабо сходится к $y$ в пространстве $L^{n}[a, b]$ при $i \rightarrow \infty$. Согласно [5] для любого $i=$ $1,2, \ldots$ сушествует такая $\widetilde{y}_{i} \in(\operatorname{co} \Phi)(x)$, для которой справедливо равенство

$$
\left\|y_{i}-\widetilde{y}_{i}\right\|_{L^{n}[a, b]}=\rho_{L^{n}[a, b]}\left[y_{i} ;(\operatorname{co} \Phi)(x)\right] .
$$

Согласно определению последовательности $\widetilde{y}_{i}, i=1,2, \ldots$, для любого $i=1,2, \ldots$ получаем соотношение

$$
\left\|y_{i}-\widetilde{y}_{i}\right\|_{L^{n}[a, b]} \leqslant h_{L^{n}[a, b]}^{+}\left[(\operatorname{co} \Phi)\left(x_{i}\right) ;(\operatorname{co} \Phi)(x)\right]
$$

из которого следует, что $\lim _{i \rightarrow \infty}\left\|y_{i}-\widetilde{y}_{i}\right\|_{L^{n}[a, b]}=0$. Поэтому последовательность $\widetilde{y}_{i}$ слабо сходится к $y$ в пространстве $L^{n}[a, b]$ при $i \rightarrow \infty$. Так как множество $($ со $\Phi)(x)$ замкнуто в слабой топологии пространства $L^{n}[a, b]$ (см. [8]), то $y \in(\operatorname{co} \Phi)(x)$. Лемма доказана.

Из леммы 5 и замечания 8 вытекает

СЛЕДСТВИЕ 2. Если отображсение $\Phi: C^{n}[a, b] \rightarrow \Pi\left[L^{n}[a, b]\right]$ непрерывно, то отображение со $\Phi: C^{n}[a, b] \rightarrow \Omega\left(\Pi\left[L^{n}[a, b]\right]\right)$ ослабленно замкнуто.

ЛЕмма 6. Пусть многозначное отображсение со $\Phi: C^{n}[a, b] \rightarrow \Omega\left(\Pi\left[L^{n}[a, b]\right]\right)$ полунепрерывно сверху по Хаусдорфу. Тогда отобрахсение со Ф переводит каждое предкомпактное множество пространства $C^{n}[a, b]$ в слабо предкомпактное множество пространства $L^{n}[a, b]$.

ДокАЗАТЕЛЬСТво. Пусть $K$ - предкомпактное множество пространства $C^{n}[a, b]$. Покажем, что $($ со $\Phi)(K)$ - слабо предкомпактное множество пространства $L^{n}[a, b]$. Пусть $z_{i} \in(\operatorname{co} \Phi)(K), i=1,2, \ldots$. Докажем, что последовательность $z_{i}, i=1,2, \ldots$, содержит слабо сходящуюся в пространстве $L^{n}[a, b]$ подпоследовательность. Пусть последовательность $x_{i}(\in K), i=1,2, \ldots$, такова, что $z_{i} \in(\operatorname{co} \Phi)\left(x_{i}\right)$. Так как $K-$ предкомпактное множество, то, не уменьшая обшности, можно считать, что $x_{i} \rightarrow x$ в пространстве $C^{n}[a, b]$ при $i \rightarrow \infty$. Для каждого $i=1,2, \ldots$ определим функцию $\widetilde{z}_{i} \in(\operatorname{co} \Phi)(x)$ равенством

$$
\left\|z_{i}-\widetilde{z}_{i}\right\|_{L^{n}[a, b]}=\rho_{L^{n}[a, b]}\left[z_{i} ;(\operatorname{co} \Phi)(x)\right] .
$$

Поэтому из полунепрерьвности сверху отображения со $\Phi: C^{n}[a, b] \rightarrow \Omega\left(\Pi\left[L^{n}[a, b]\right]\right)$ вытекает равенство

$$
\lim _{i \rightarrow \infty}\left\|z_{i}-\widetilde{z}_{i}\right\|_{L^{n}[a, b]}=0 .
$$

Так как последовательность $\widetilde{z}_{i} \in(\operatorname{co} \Phi)(x), i=1,2, \ldots$, то она слабо компактна в $L^{n}[a, b]$. Не уменьшая общности, можно считать, что $z_{i} \rightarrow z$ слабо в пространстве $L^{n}[a, b]$ при $i \rightarrow \infty$. Отсюда согласно равенству (43) $z_{i} \rightarrow z$ слабо в пространстве $L^{n}[a, b]$ при $i \rightarrow \infty$. Поэтому образ $(\operatorname{co} \Phi)(K)$ - слабо предкомпактное множество пространства $L^{n}[a, b]$. Лемма доказана. 
ТеОрема 4. Пусть отображение $V: L^{n}[a, b] \rightarrow C^{n}[a, b]$ непрерывно, а многозначные отображсения

$$
\Psi: C^{n}[a, b] \rightarrow \operatorname{comp}\left[C^{n}[a, b]\right], \quad \operatorname{co} \Phi: C^{n}[a, b] \rightarrow \Omega\left(\Pi\left[L^{n}[a, b]\right]\right)
$$

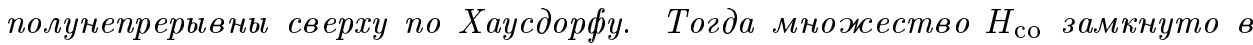
пространстве $C^{n}[a, b]$.

ДокАЗАтЕЛьство. Пусть $H_{\text {со }} \neq \varnothing$, и пусть последовательность $x_{i} \in H_{\text {со }}, i=$ $1,2, \ldots$, такова, что $x_{i} \rightarrow x$ в $C^{n}[a, b]$ при $i \rightarrow \infty$. Пусть $v_{i} \in \Psi\left(x_{i}\right), z_{i} \in(\operatorname{co} \Phi)\left(x_{i}\right)$, $i=1,2, \ldots$, удовлетворяют равенству

$$
x_{i}=v_{i}+V z_{i}
$$

Далее, покажем, что, не уменьшая общности, можно считать, что последовательность $v_{i}, i=1,2, \ldots$, является сходящейся в пространстве $C^{n}[a, b]$. Действительно, пусть для любого $i=1,2, \ldots$ функция $\widetilde{v}_{i} \in \Psi(x)$ определена равенством

$$
\left\|v_{i}-\widetilde{v}_{i}\right\|_{C^{n}[a, b]}=\rho_{C^{n}[a, b]}\left[v_{i} ; \Psi(x)\right]
$$

Тогда из определения последовательности $v_{i}, i=1,2, \ldots$, для любого $i=1,2, \ldots$ следует неравенство

$$
\left\|v_{i}-\widetilde{v}_{i}\right\|_{C^{n}[a, b]} \leqslant h_{C^{n}[a, b]}^{+}\left[\Psi\left(x_{i}\right) ; \Psi(x)\right] .
$$

Отсюда и из полунепрерывности сверху по Хаусдорфу отображения $\Psi$ получаем соотношение

$$
\lim _{i \rightarrow \infty}\left\|v_{i}-\widetilde{v}_{i}\right\|_{C^{n}[a, b]}=0 .
$$

Так как множество $\Psi(x)$ компактно в $C^{n}[a, b]$, то последовательность $\widetilde{v}_{i}, i=1,2, \ldots$, компактна. Поэтому, не уменьшая общности, можно считать, что $\lim _{i \rightarrow \infty} \widetilde{v}_{i}=v$, где $v \in \Psi(x)$. Из равенства (45) следует, что и $\lim _{i \rightarrow \infty} v_{i}=v$.

Далее, согласно лемме 6 последовательность $z_{i} \in(\operatorname{co} \Phi)\left(x_{i}\right), i=1,2, \ldots$, слабо компактна в пространстве $L^{n}[a, b]$. Поэтому, не уменьшая общности, можно считать, что $z_{i} \rightarrow z$ слабо в $L^{n}[a, b]$ при $i \rightarrow \infty$, в силу леммы $5 z \in(\operatorname{co} \Phi)(x)$. Из (44) и сделанных вьше предположений следует, что $V z_{i} \rightarrow V z$ в пространстве $C^{n}[a, b]$ при $i \rightarrow \infty$. Поэтому, переходя в (44) к пределу при $i \rightarrow \infty$, получаем соотноше-

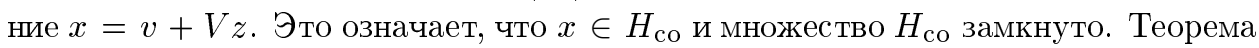
доказана.

ОПРЕДЕЛЕНИЕ. Будем говорить, что отображения

$$
V: L^{n}[a, b] \rightarrow C^{n}[a, b], \quad \Psi: C^{n}[a, b] \rightarrow \operatorname{comp}\left[C^{n}[a, b]\right], \quad \Phi: C^{n}[a, b] \rightarrow \Pi\left[L^{n}[a, b]\right]
$$

обладают свойством $\mathrm{B}$, если эти отображения обладают свойством $\mathrm{A}^{*}$ и оператор $V$ переводит каждое слабо предкомпактное в $L^{n}[a, b]$ множество в предкомпактное множество пространства $C^{n}[a, b]$.

Пусть $H$ - множество решений включения (7). 
Теорема 5. Пусть отображения

$V: L^{n}[a, b] \rightarrow C^{n}[a, b], \quad \Psi: C^{n}[a, b] \rightarrow \operatorname{comp}\left[C^{n}[a, b]\right], \quad \Phi: C^{n}[a, b] \rightarrow \Pi\left[L^{n}[a, b]\right]$

обладают свойством В. Тогда множество $H \neq \varnothing$ и справедливо равенство

$$
\bar{H}=H_{\mathrm{co}},
$$

әде $\bar{H}$ - замыкание в пространстве $C^{n}[a, b]$ множества $H$.

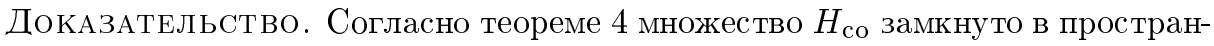
стве $C^{n}[a, b]$. Так как $H \subset H_{\text {со }}$, то из замкнутости множества $H_{\text {со вытекает, что }}$ для доказательства равенства (46) достаточно показать, что $H_{\text {со }} \subset \bar{H}$. Докажем это вложение. Пусть $x \in H_{\text {со }}$. Согласно теореме $2 x \in \mathscr{H}$. Это означает, что найдутся элемент $p \in \Psi(x)$ и последовательность $g_{i} \in \Phi(x), i=1,2, \ldots$, такие, что последовательность $y_{i}=p+V g_{i} \rightarrow x$ в $C^{n}[a, b]$ при $i \rightarrow \infty$. Тогда согласно свойству В из соотношения (12) для любого $i=1,2, \ldots$ и любого измеримого множества $\mathscr{U} \subset[a, b]$ получаем оценку

$$
\rho_{L^{n}(\mathscr{U})}\left[g_{i} ; \Phi\left(y_{i}\right)\right] \leqslant h_{L^{n}(\mathscr{U})}\left[\Phi(x) ; \Phi\left(y_{i}\right)\right] \leqslant\left\|\Gamma\left(Z\left(x-y_{i}\right)\right)\right\|_{L^{1}(\mathscr{U})} .
$$

Далее, пусть для любого $i=1,2, \ldots \quad r_{i} \in \Psi\left(y_{i}\right)$ удовлетворяет равенству

$$
\left\|p-r_{i}\right\|_{C^{n}[a, b]}=\rho_{C^{n}[a, b]}\left[p ; \Psi\left(y_{i}\right)\right] .
$$

Поэтому из непрерывности отображения $\Psi$ следует, что $r_{i} \rightarrow p$ в $C^{n}[a, b]$ при $i \rightarrow \infty$.

Пусть непрерывная функция $\nu_{i}:[a, b] \rightarrow[0, \infty), i=1,2, \ldots$, определена соотношением

$$
\nu_{i}(t)=\int_{a}^{b}|V(t, s)| \Gamma\left(Z\left(x-y_{i}\right)\right)(s) d s+\left|p(t)-r_{i}(t)\right| .
$$

Так как $y_{i} \rightarrow x$ в $C^{n}[a, b]$ при $i \rightarrow \infty$, то $\nu_{i} \rightarrow 0$ в $C^{n}[a, b]$ при $i \rightarrow \infty$. Далее, в силу свойства В и теоремы 1 для каждой тройки функций $\left(y_{i}, r_{i}, g_{i}\right), i=1,2, \ldots$, найдется такое решение $x_{i} \in H$, для которого для любого $t \in[a, b]$ справедливо неравенство $\left|x_{i}(t)-y_{i}(t)\right| \leqslant \xi_{i}(t)$, где $\xi_{i}$ - сумма ряда (17) для функции $\nu_{i}$, определенной равенством (47). Согласно свойству В сумма ряда (17) непрерьвна в нуле относительно функции $\nu$, поэтому $\xi_{i} \rightarrow 0$ в $C^{n}[a, b]$ при $i \rightarrow \infty$. А из этого вытекает, что $x_{i} \rightarrow x$ в $C^{n}[a, b]$ при $i \rightarrow \infty$. Это означает, что $x \in \bar{H}$ и, следовательно, $H_{\text {co }} \subset \bar{H}$. Теорема доказана.

ЗАмечАниЕ 9. Из рассмотренного приложения к теореме 1 вытекает, что из теоремы 5 следует теорема А. Ф. Филиппова (см. [11], [12]) для дифференциального включения с запаздыванием.

ЗАмечание 10. Отметим, что выполнение равенства (46) в последнее время называют принципом плотности (см. [5], [16]). Принцип плотности вьполняется не всегда. Это доказывает пример Плиса (см. [14], [20]). 
ЗАмЕчАНИЕ 11 . Отметим, что теорема 5 в отличие от [5] не предполагает выпуклозначности отображения $\Psi: C^{n}[a, b] \rightarrow \operatorname{comp}\left[C^{n}[a, b]\right]$, и поэтому теорема 5 уточняет аналогичный результат из [5].

ЗАмЕчАНИЕ 12. Вопрос о плотности множества решений для задачи Коши дифференциального включения с невыпуклой правой частью в множестве решений "овьпукленного" включения был поставлен и решен А.Ф. Филипповьм (см. [12]). Распространением и уточнением теоремы А.Ф. Филиппова для более общих случаев занимались многие авторы (см., например, [21]-[29]). Для периодических решений и для краевых задач дифференциальных включений эта задача впервые решена в работах [3]-[6], [10], [17], [30]. Отметим, что свойство плотности множества решений, как отмечено Пианижиани (см. [28]), является фундаментальным свойством в теории дифференциальных включений с невьпуклой правой частью. Кроме того, как показано в работах [16], [31], [32], это свойство играет важную роль в теории устойчивости и аппроксимации дифференциальных включений, так как оно является необходимым и достаточным условием непрерывной зависимости множества решений относительно внутренних и внешних возмушений (см. [31]-[33]). Поэтому оно занимает центральное место в теории и приложениях дифференциальных включений. Следовательно, вопрос о плотности множества решений имеет важное значение и в теории возмушенных включений, так как дифференциальное включение является частным случаем возмущенного включения.

Определим отображение ext $\Delta:[a, b] \times C^{n}[a, b] \rightarrow \operatorname{comp}\left[\mathbb{R}^{n}\right]$ равенством

$$
(\operatorname{ext} \Delta)(t, x)=\overline{\operatorname{ext}}(\operatorname{co}(\Delta(t, x)))
$$

Отметим, что для каждого $x \in C^{n}[a, b]$ отображение $(\operatorname{ext} \Delta)(\cdot, x)$ измеримо [18] и ограничено суммируемой функцией.

Рассмотрим отображение $\operatorname{ext} \Phi: C^{n}[a, b] \rightarrow \Pi\left[L^{n}[a, b]\right]$, заданное соотношением

$$
(\operatorname{ext} \Phi)(x)=\left\{y \in L^{n}[a, b]: y(t) \in \overline{\operatorname{ext}}(\operatorname{co}(\Delta(t, x))) \text { при п.в. } t \in[a, b]\right\} .
$$

Из сделанного выше замечания относительно названия отображения $\Delta:[a, b] \times$ $C^{n}[a, b] \rightarrow \operatorname{comp}\left[\mathbb{R}^{n}\right]$, а также из определения отображения $\operatorname{ext} \Phi: C^{n}[a, b] \rightarrow$ $\Pi\left[L^{n}[a, b]\right]$ вытекает, что для многозначного оператора Немыцкого $\mathscr{N}: C^{n}[a, b] \rightarrow$ $\Pi\left[L^{n}[a, b]\right]$, порожденного функцией $F:[a, b] \times \mathbb{R}^{n} \rightarrow \operatorname{comp}\left[\mathbb{R}^{n}\right]$, отображение $\operatorname{ext} \mathscr{N}: C^{n}[a, b] \rightarrow \Pi\left[L^{n}[a, b]\right]$ есть оператор Немыцкого, порожденный функцией $\operatorname{ext} F:[a, b] \times \mathbb{R}^{n} \rightarrow \operatorname{comp}\left[\mathbb{R}^{n}\right]$, определенной равенством

$$
(\operatorname{ext} F)(t, x)=\overline{\operatorname{ext}}(\operatorname{co}(F(t, x))) .
$$

ЗАмечАНИЕ 13. Отметим, что для любого $x \in C^{n}[a, b]$ множество $(\operatorname{ext} \Phi)(x)$ обладает свойством

$$
\overline{\mathrm{co}}((\operatorname{ext} \Phi)(x))=\overline{\mathrm{co}}(\Phi(x)) .
$$

Причем множество $(\operatorname{ext} \Phi)(x)$ минимальное по включению среди непустых замкнутых в пространстве $L^{n}[a, b]$ выпуклых по переключению подмножеств, принадлежащих множеству $\Phi(x)$ и удовлетворяющих равенству (50). 
Рассмотрим в пространстве $C^{n}[a, b]$ включение

$$
x \in \Psi(x)+V(\operatorname{ext} \Phi)(x),
$$

где отображение ext $\Phi: C^{n}[a, b] \rightarrow \Pi\left[L^{n}[a, b]\right]$ определено равенством (49). Пусть $\mathscr{H}_{\text {ext }}, H_{\text {ext }}$ - множества всех квазирешений и решений включения $(51)$ соответственно.

Из равенства (50) и теоремы 2 вытекает

СЛЕДСТВИЕ 3. Пусть линейный непрерьвный оператор $V: L^{n}[a, b] \rightarrow C^{n}[a, b]$, определенный равенством (8), переводит кажсдое слабо компактное в $L^{n}[a, b]$ множество в предкомпактное мнохсество пространства $C^{n}[a, b]$. Тогда справедливо равенство $H_{\text {со }}=\mathscr{H}_{\text {ext }}$.

Ниже доказана теорема о так называемом “бэнг-бэнг” принципе возмущенного включения (7). Отметим, что “бэнг-бэнг” принцип играет важную роль в теории управления, поскольку позволяет “упростить" систему управления путем сужения множества допустимых значений управления. При этом замыкания в пространстве непрерывных функций множеств траекторий первоначальной системы управления и упрошенной совпадают. Таким образом, "возможности” упрошенной системы управления в этом случае сравнимы с первоначальной. В то же время "управлять" упрощенной системой гораздо проше, так как множество допустимых управлений существенно сужается (во многих случаях допустимое управление можно осушествлять простьми с точки зрения вычислений функциями, иногда их называют релейнымми).

ОПРЕДЕЛЕНИЕ. Будем говорить (см. [5], [17], [21], [22]), что для включения (7) выполняется “бэнг-бәнг” приниип, если выполняются равенства

$$
\bar{H}_{\mathrm{ext}}=\bar{H}=H_{\mathrm{co}},
$$

где $\bar{H}_{\text {ext }}, \bar{H}$ - замькания множеств $H_{\text {ext }}, H$ в пространстве $C^{n}[a, b]$.

ОПРЕДЕЛЕНИЕ. Будем говорить (см. [5]), что множество $H_{\text {со }}$ разложимо по многозначнылм отображсениям $\Psi, \operatorname{co} \Phi$, или просто разложсимо, если каждое

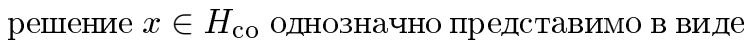

$$
x=v+V z,
$$

где $v \in \Psi(x), z \in(\operatorname{co} \Phi)(x)$.

ОПРЕДЕЛЕНИЕ. Будем говорить, что отображения

$$
\begin{aligned}
V: L^{n}[a, b] \rightarrow & C^{n}[a, b], \quad \Psi: C^{n}[a, b] \rightarrow \operatorname{comp}\left[C^{n}[a, b]\right], \\
& \operatorname{co} \Phi: C^{n}[a, b] \rightarrow \Omega\left(\Pi\left[L^{n}[a, b]\right]\right)
\end{aligned}
$$

обладают свойством $\mathrm{B}^{*}$, если эти отображения обладают свойством В, ядро

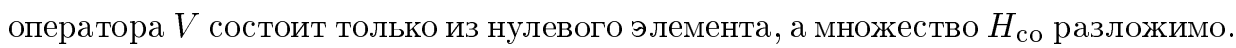

Далее, определим отображение $\mathscr{P}: H_{\text {со }} \rightarrow C^{n}[a, b]$ равенством

$$
\mathscr{P}(x)=v,
$$

где функция $v \in C^{n}[a, b]$ определена представлением (53).

Обозначим через $V^{-1}(y)$ прообраз элемента $y \in C^{n}[a, b]$ оператора $V: L^{n}[a, b] \rightarrow$ $C^{n}[a, b]$, определенного равенством (8). 
ЛЕММА 7. Пусть отображения

$V: L^{n}[a, b] \rightarrow C^{n}[a, b], \quad \Psi: C^{n}[a, b] \rightarrow \operatorname{comp}\left[C^{n}[a, b]\right], \quad \Phi: C^{n}[a, b] \rightarrow \Pi\left[L^{n}[a, b]\right]$

обладают свойством $\mathrm{B}^{*}$. Тогда

1) отобрахсение $\mathscr{P}: H_{\mathrm{co}} \rightarrow C^{n}[a, b]$, определенное равенством (54), непрерьвно;

2) суперпозиция $V^{-1}(I-\mathscr{P}): H_{\text {со }} \rightarrow L^{n}[a, b]$ непрерывна в слабой топологии пространства $L^{n}[a, b]$ и локально компактна в этой топологии, где оператор $I: C^{n}[a, b] \rightarrow C^{n}[a, b]$ - тождественный оператор.

ДокАЗАТЕЛЬство. Пусть последовательность $\left\{x_{i}\right\} \subset H_{\text {со }}$. Это означает, что для любого $i=1,2, \ldots$ сушествует $z_{i} \in \overline{\mathrm{co}}\left(\Phi\left(x_{i}\right)\right)$, для которого вьполняется равенство

$$
x_{i}=\mathscr{P}\left(x_{i}\right)+V z_{i} .
$$

Согласно определению отображения $\mathscr{P}: H_{\text {со }} \rightarrow C^{n}[a, b]$ для любого $i=1,2, \ldots$ имеет место вложение $\mathscr{P}\left(x_{i}\right) \in \Psi\left(x_{i}\right)$. Из равенства (53) вытекает, что для любого $i=1,2, \ldots$ справедливо соотношение $z_{i}=V^{-1}\left(x_{i}-\mathscr{P}\left(x_{i}\right)\right)$. Пусть теперь $x_{i} \rightarrow x$ в пространстве $C^{n}[a, b]$ при $i \rightarrow \infty$. Покажем, что последовательность $\left\{\mathscr{P}\left(x_{i}\right)\right\}$ сходится в пространстве $C^{n}[a, b]$, а последовательность $\left\{z_{i}\right\}$ имеет предел в слабой топологии пространства $L^{n}[a, b]$. Действительно, предположим противное. Тогда в силу компактности последовательностей $\left\{\mathscr{P}\left(x_{i}\right)\right\}$ и $\left\{z_{i}\right\}$ в соответствующих топологиях найдутся две пары последовательностей $\left\{\mathscr{P}\left(x_{i_{j}^{1}}\right)\right\},\left\{z_{i_{j}^{1}}\right\}$ и $\left\{\mathscr{P}\left(x_{i_{j}^{2}}\right)\right\},\left\{z_{i_{j}^{2}}\right\}$, обладающие свойством: $\mathscr{P}\left(x_{i_{j}^{1}}\right) \rightarrow v_{1}$, а $\mathscr{P}\left(x_{i_{j}^{2}}\right) \rightarrow v_{2}$ в пространстве $C^{n}[a, b]$ и $z_{i_{j}^{1}} \rightarrow z_{1}$, а $z_{i_{j}^{2}} \rightarrow z_{2}$ слабо в пространстве $L^{n}[a, b]$ при $j \rightarrow \infty$, причем $v_{1} \neq v_{2}$ и $z_{1} \neq z_{2}$. Переходя в равенствах (55) к пределу при $j \rightarrow \infty$ по соответствуюшим последовательностям, получаем соотношения

$$
x=v_{1}+V z_{1}, \quad x=v_{2}+V z_{2} .
$$

Так как отображение $\Psi: C^{n}[a, b] \rightarrow \operatorname{comp}\left[C^{n}[a, b]\right]$ непрерьвно по Хаусдорфу, то $v_{1}, v_{2} \in \Psi(x)$. В силу леммы 5 отображение со $\Phi: C^{n}[a, b] \rightarrow \Omega\left(\Pi\left[L^{n}[a, b]\right]\right)$ ослабленно замкнуто, поэтому $z_{1}, z_{2} \in \overline{\mathrm{co}}(\Phi(x))$. Таким образом, $x$ - решение овыпукленного включения (38), но равенства (56) противоречат разложимости множества $H_{\text {со }}$. Следовательно, последовательности $\left\{\mathscr{P}\left(x_{i}\right)\right\}$ и $\left\{z_{i}\right\}$ имеют пределы в соответствующих топологиях, причем $\lim _{i \rightarrow \infty} \mathscr{P}\left(x_{i}\right)=\mathscr{P}(x)$, a $\lim _{i \rightarrow \infty} z_{i}=$ $V^{-1}(x-\mathscr{P}(x))$. Таким образом, отображение $\mathscr{P}: H_{\text {со }} \rightarrow C^{n}[a, b]$ непрерывно в пространстве $C^{n}[a, b]$, а отображение $V^{-1}(I-\mathscr{P}): H_{\text {со }} \rightarrow L^{n}[a, b]$ непрерывно в слабой топологии пространства $L^{n}[a, b]$. Так как согласно неравенству (12) для любого измеримого множества $\mathscr{U} \subset[a, b]$ имеет место оценка

$$
\|\Phi(x)\|_{L^{n}(\mathscr{U})} \leqslant\|\Phi(0)\|_{L^{n}(\mathscr{U})}+\|\Gamma Z(x)\|_{L^{1}(\mathscr{U})},
$$

то из неравенства (57) вытекает, что для каждого ограниченного множества пространства $C^{n}[a, b]$ образ отображения со $\Phi: C^{n}[a, b] \rightarrow\left(\Pi\left[L^{n}[a, b]\right]\right)$ ограничен суммируемой функцией. Поэтому суперпозиция $V^{-1}(I-\mathscr{P}): H_{\text {со }} \rightarrow L^{n}[a, b]$ локально компактна в слабой топологии пространства $L^{n}[a, b]$. Лемма доказана. 
ЛЕмма 8. Пусть $U$ - замкнутое множество пространства $C^{n}[a, b], u$ пусть отображение $\Xi: U \rightarrow L^{n}[a, b]$ непрерывно в слабой топологии пространства $L^{n}[a, b]$ и локально компактно в әтой топологии. Тогда функционал $\eta: U \rightarrow \mathbb{R}^{1}$, определенный равенством

$$
\eta(x)=\|\Xi(x)\|_{L^{n}[a, b]},
$$

полунепрерывен снизу на $U$.

ДоказАТЕЛЬСтво. Покажем, что множество $U(\tau)=\{x \in U: \eta(x) \leqslant \tau\}$ замкнуто для любого $\tau \geqslant 0$. Пусть последовательность $x_{i} \in U(\tau), i=1,2, \ldots$, такова, что $x_{i} \rightarrow x$ в пространстве $C^{n}[a, b]$ при $i \rightarrow \infty$. Тогда $z_{i}=\Xi\left(x_{i}\right) \rightarrow \Xi(x)=z_{0}$ слабо в пространстве $L^{n}[a, b]$ при $i \rightarrow \infty$. Пусть для любого $i=1,2, \ldots$ функция $\alpha_{i} \in L^{1}[a, b]$ определена равенством $\alpha_{i}(t)=\left|z_{i}(t)\right|, t \in[a, b]$. Не уменьшая общности, можно считать, что последовательность $\alpha_{i}$ сходится слабо к элементу $\alpha \in L^{1}[a, b]$. Тогда согласно [8] для каждого $m=1,2, \ldots$ найдутся такие числа $i(m), \lambda_{j}^{m} \geqslant 0, j=1,2, \ldots, i(m)$, что $\sum_{j=1}^{i(m)} \lambda_{j}^{m}=1$ и последовательность $\left\{\beta_{m}=\sum_{j=1}^{i(m)} \lambda_{j}^{m} \alpha_{j+m}\right\}$ сходится к $\alpha$ в пространстве $L^{1}[a, b]$. Ниже, не уменьшая обшности, будем считать, что $\beta_{m} \rightarrow \alpha$ почти всюду на $[a, b]$ при $m \rightarrow \infty$. Для любого $m=1,2, \ldots$ функцию $y_{m} \in L^{n}[a, b]$ определим равенством

$$
y_{m}(t)=\sum_{j=1}^{i(m)} \lambda_{j}^{m} z_{j+m}(t) .
$$

Из определения функции $y_{m}$ при почти всех $t \in[a, b]$ получаем оценку

$$
\left|y_{m}(t)\right| \leqslant \sum_{j=1}^{i(m)} \lambda_{j}^{m}\left|z_{j+m}(t)\right|=\beta_{m}(t) .
$$

Таким образом, из неравенства (60) при почти всех $t \in[a, b]$ следует включение

$$
y_{m}(t) \in \overline{B\left[0 ; \beta_{m}(t)\right]} \text {. }
$$

Пусть для каждого $m=1,2, \ldots$ функция $\widetilde{y}_{m} \in L^{n}[a, b]$ при почти всех $t \in[a, b]$ удовлетворяют включению $\widetilde{y}_{m}(t) \in \overline{B[0 ; \alpha(t)]}$ и соотношению

$$
\left|\widetilde{y}_{m}(t)-y_{m}(t)\right|=\rho\left[y_{m}(t) ; \overline{B[0 ; \alpha(t)]}\right] .
$$

Из определения функций $\widetilde{y}_{m}$ и включения (61) следует, что при почти всех $t \in[a, b]$ $\left|\widetilde{y}_{m}(t)-y_{m}(t)\right| \rightarrow 0$ при $m \rightarrow \infty$. Так как из равенства (59) вытекает, что $y_{m} \rightarrow z_{0}$ слабо в $L^{n}[a, b]$ при $m \rightarrow \infty$, то и $\widetilde{y}_{m} \rightarrow z_{0}$ слабо в $L^{n}[a, b]$ при $m \rightarrow \infty$. Поэтому при почти всех $t \in[a, b]$ справедливо включение $z_{0}(t) \in \overline{B[0 ; \alpha(t)]}$, т.е. для функции $z_{0}$ при почти всех $t \in[a, b]$ вьполняется оценка $\left|z_{0}(t)\right| \leqslant \alpha(t)$. Отсюда согласно определению функций $\alpha_{i}, i=1,2, \ldots$, получаем соотношения

$$
\int_{a}^{b}\left|z_{0}(t)\right| d t \leqslant \int_{a}^{b} \alpha(t) d t=\lim _{i \rightarrow \infty} \int_{a}^{b} \alpha_{i}(t) d t \leqslant \tau .
$$


Следовательно, $\eta(x) \leqslant \tau$, а это означает, что множество $U(\tau)$ замкнуто, и поэтому функционал $\eta: U \rightarrow \mathbb{R}^{1}$, определенный равенством (58), полунепрерывен снизу. Лемма доказана.

Рассмотрим функционал $W: C^{n}[a, b] \rightarrow \mathbb{R}^{1}$, заданный равенством

$$
W(x)=W(x, g(x))=\rho_{L^{n}[a, b]}[g(x) ;(\operatorname{ext} \Phi)(x)]
$$

где отображение $g: C^{n}[a, b] \rightarrow L^{n}[a, b]$, а оператор ext $\Phi: C^{n}[a, b] \rightarrow \Pi\left[L^{n}[a, b]\right]$ определен равенством (49).

ЛЕмма 9.Пусть найдется изотонный непрерывный оператор $\Gamma: C_{+}^{1}[a, b] \rightarrow$ $L^{1}[a, b]$, удовлетворяющий условию $\Gamma(0)=0$, такой, что для любъх $x, y \in$ $C^{n}[a, b]$ и любого измеримого множества $\mathscr{U} \subset[a, b]$ для отображения $\Phi:$ $C^{n}[a, b] \rightarrow \Pi\left[L^{n}[a, b]\right]$ выполняется неравенство (12). Далее, пусть отображсение $g: C^{n}[a, b] \rightarrow L^{n}[a, b]$ непрерывно. Тогда функиионал $W: C^{n}[a, b] \rightarrow \mathbb{R}^{1}$, определенный равенством (62), полунепрерывен сверху на пространстве $C^{n}[a, b]$.

ДоказАтельство. Прежде всего покажем, что отображение ext $\Phi: C^{n}[a, b] \rightarrow$ $\Pi\left[L^{n}[a, b]\right]$, определенное равенством (49), полунепрерывно снизу. Пусть $x \in$ $C^{n}[a, b]$, и пусть последовательность $x_{i} \in C^{n}[a, b], i=1,2, \ldots$, сходится к $x$ в пространстве $C^{n}[a, b]$ при $i \rightarrow \infty$. Так как непрерьвньй оператор $\Gamma: C_{+}^{1}[a, b] \rightarrow L^{1}[a, b]$ изотонный и удовлетворяет условию $\Gamma(0)=0$, то из неравенства $(12)$ и замечания 8 для почти всех $t \in[a, b]$ выполняется соотношение

$$
\lim _{i \rightarrow \infty} h\left[\operatorname{co}\left(\Delta\left(t, x_{i}\right)\right) ; \operatorname{co}(\Delta(t, x))\right]=0
$$

где многозначное отображение $\Delta:[a, b] \times C^{n}[a, b] \rightarrow \operatorname{comp}\left[\mathbb{R}^{n}\right]$ определено равенством (39). Отсюда согласно теоремам Егорова и Лузина (см. [9]) для любого $\delta>0$ найдется такое замкнутое множество $J_{\delta} \subset[a, b]$, удовлетворяющее неравенству $\mu\left([a, b] \backslash J_{\delta}\right)<\delta$, что при всех $i=1,2, \ldots$ отображение $h\left[\operatorname{co}\left(\Delta\left(\cdot, x_{i}\right)\right) ; \operatorname{co}(\Delta(\cdot, x))\right]$ непрерывно на $J_{\delta}$ и равенство (63) вьполняется равномерно на $J_{\delta}$. Пусть $K=$ $\left\{x_{i}, i=1,2, \ldots ; x\right\}$. Тогда отображение со $\Delta: J_{\delta} \times K \rightarrow \operatorname{comp}\left[\mathbb{R}^{n}\right]$ непрерывно. Следовательно, согласно [18] отображение ext $\Delta: J_{\delta} \times K \rightarrow \operatorname{comp}\left[\mathbb{R}^{n}\right]$, определенное равенством (48), полунепрерьвно снизу. Отметим, что для любого $i=1,2, \ldots$ и для любого множества $J_{\delta} \subset[a, b]$ справедливо соотношение

$$
\begin{aligned}
& h_{L^{n}[a, b]}^{+}\left[(\operatorname{ext} \Phi)(x) ;(\operatorname{ext} \Phi)\left(x_{i}\right)\right] \\
& \quad=h_{L^{n}\left([a, b] \backslash J_{\delta}\right)}^{+}\left[(\operatorname{ext} \Phi)(x) ;(\operatorname{ext} \Phi)\left(x_{i}\right)\right]+h_{L^{n}\left(J_{\delta}\right)}^{+}\left[(\operatorname{ext} \Phi)(x) ;(\operatorname{ext} \Phi)\left(x_{i}\right)\right] .
\end{aligned}
$$

Так как согласно неравенству (12) образ $\Phi(K)$ ограничен суммируемой функцией, то для любого $\varepsilon>0$ сушествует такое замкнутое множество $J_{\delta(\varepsilon)} \subset[a, b]$, для которого при всех $i=1,2, \ldots$ имеет место оценка

$$
h_{L^{n}\left([a, b] \backslash J_{\delta(\varepsilon)}\right)}^{+}\left[(\operatorname{ext} \Phi)(x) ;(\operatorname{ext} \Phi)\left(x_{i}\right)\right]<\varepsilon .
$$


Из определения оператора ext $\Phi: C^{n}[a, b] \rightarrow \Pi\left[L^{n}[a, b]\right]$ (см. равенства (48), (49)) и полунепрерывности снизу отображения ext $\Delta: J_{\delta(\varepsilon)} \times K \rightarrow \operatorname{comp}\left[\mathbb{R}^{n}\right]$ следует, что для любого $\varepsilon>0$ найдется число $i_{0}$ такое, что при всех $i \geqslant i_{0}$ вьполняется неравенство

$$
h_{L^{n}\left(J_{\delta(\varepsilon)}\right)}^{+}\left[(\operatorname{ext} \Phi)(x) ;(\operatorname{ext} \Phi)\left(x_{i}\right)\right]<\varepsilon .
$$

Поэтому из равенства (64) и оценок $(65),(66)$ при всех $i \geqslant i_{0}$ имеет место соотношение

$$
h_{L^{n}[a, b]}^{+}\left[(\operatorname{ext} \Phi)(x) ;(\operatorname{ext} \Phi)\left(x_{i}\right)\right] \leqslant 2 \varepsilon .
$$

Таким образом, отображение $\operatorname{ext} \Phi: C^{n}[a, b] \rightarrow \Pi\left[L^{n}[a, b]\right]$ полунепрерьвно снизу.

Далее, покажем, что функционал $W: C^{n}[a, b] \rightarrow \mathbb{R}^{1}$, определенный равенством (62), полунепрерывен сверху. Пусть последовательность $x_{i}, i=1,2, \ldots$, сходится к $x$ в пространстве $C^{n}[a, b]$ при $i \rightarrow \infty$. И пусть измеримая функция $u:[a, b] \rightarrow \mathbb{R}^{n}$ удовлетворяет при почти всех $t \in[a, b]$ равенству

$$
|g(x)(t)-u(t)|=\rho[g(x) ;(\operatorname{ext} \Delta)(t, x)],
$$

где отображение ext $\Delta:[a, b] \times C^{n}[a, b] \rightarrow \operatorname{comp}\left[\mathbb{R}^{n}\right]$ определено соотношением (48). Согласно определению отображения $\operatorname{ext} \Phi: C^{n}[a, b] \rightarrow \Pi\left[L^{n}[a, b]\right]$ (см. (49)) $u \in$ $(\operatorname{ext} \Phi)(x)$. Поэтому по доказанному для функции $u$ найдутся такие функции $u_{i} \in$ $(\operatorname{ext} \Phi)\left(x_{i}\right), i=1,2, \ldots$, что $u_{i} \rightarrow u$ в пространстве $L^{n}[a, b]$ при $i \rightarrow \infty$. В силу определения функционала $W: C^{n}[a, b] \rightarrow \mathbb{R}^{1}$ (см. (62)) для любого $i=1,2, \ldots$ выполняются оценки $W\left(x_{i}\right) \leqslant\left\|g\left(x_{i}\right)-u_{i}\right\|_{L^{n}[a, b]}$. Отсюда согласно равенству (67) получаем соотношение

$$
\lim _{i \rightarrow \infty} \sup W\left(x_{i}\right) \leqslant \lim _{i \rightarrow \infty}\left\|g\left(x_{i}\right)-u_{i}\right\|_{L^{n}[a, b]}=\|g(x)-u\|_{L^{n}[a, b]}=W(x) .
$$

Лемма доказана.

Теорема 6. Пусть отображсения

$$
\begin{aligned}
V: L^{n}[a, b] \rightarrow & C^{n}[a, b], \quad \Psi: C^{n}[a, b] \rightarrow \operatorname{comp}\left[C^{n}[a, b]\right], \\
& \operatorname{co} \Phi: C^{n}[a, b] \rightarrow \Omega\left(\Pi\left[L^{n}[a, b]\right]\right)
\end{aligned}
$$

обладают свойством $\mathrm{B}^{*}$. Тогда $H_{\mathrm{ext}} \neq \varnothing$ и для включения (7) виполняется “бэнг-бәнг” принцип, т.е. справедливо равенство (52).

ДокАЗАТЕЛЬство. Из определений отображений $\operatorname{ext} \Phi: C^{n}[a, b] \rightarrow \Pi\left[L^{n}[a, b]\right]$ и со $\Phi: C^{n}[a, b] \rightarrow \Omega\left(\Pi\left[L^{n}[a, b]\right]\right)$ следует, что для любого $x \in C^{n}[a, b]$ выполняются вложения $(\operatorname{ext} \Phi)(x) \subset \Phi(x) \subset(\operatorname{co} \Phi)(x)$. Поэтому $H_{\text {ext }} \subset H \subset H_{\text {со }}$. Остается доказать, что $H_{\text {со }} \subset \overline{H_{\text {ext }}}$. Отметим, что в силу теоремы $1 H_{\text {со }} \neq \varnothing$, поэтому из последнего соотношения будет вытекать, что и $H_{\text {ext }} \neq \varnothing$.

Пусть $\left\{u_{m}\right\} \subset L^{n}[a, b]$-последовательность, плотная в образе $(\operatorname{co} \Phi)\left(H_{\mathrm{co}}\right)$. Для каждых $m, i=1,2, \ldots$ определим отображения $\rho_{m}: H_{\text {со }} \rightarrow \mathbb{R}^{1}$ и $\Phi_{m i}: H_{\mathrm{co}} \rightarrow$ $\Omega\left(L^{n}[a, b]\right)$ равенствами

$$
\rho_{m}(x)=\rho_{L^{n}[a, b]}\left[u_{m} ;(\operatorname{co} \Phi)(x)\right], \quad \Phi_{m i}=(\operatorname{co} \Phi)(x) \cap B_{L^{n}[a, b]}\left[u_{m} ; \rho_{m}(x)+i^{-1}\right] .
$$


Так как отображение со $\Phi: C^{n}[a, b] \rightarrow \Omega\left(\Pi\left[L^{n}[a, b]\right]\right)$ удовлетворяет неравенству (12), а для изотонного непрерывного оператора $\Gamma: C_{+}^{1}[a, b] \rightarrow L_{+}^{1}[a, b]$ справедливо соотношение $\Gamma(0)=0$, то оно непрерьвно. Поэтому для любого $m=1,2, \ldots$ непрерывен функционал $\rho_{m}: H_{\text {со }} \rightarrow \mathbb{R}^{1}$. Далее, покажем, для любых $m, i=$ $1,2, \ldots$ отображение $\Phi_{m i}: H_{\text {со }} \rightarrow \Omega\left(L^{n}[a, b]\right)$ полунепрерьвно снизу. Действительно, пусть последовательность $\left\{x_{j}\right\} \subset H_{\text {со }}$ сходится к $x$ в пространстве $C^{n}[a, b]$ при $j \rightarrow \infty$. Пусть $y \in \Phi_{m i}(x)$. Тогда согласно определению отображения $\Phi_{m i}: H_{\text {со }} \rightarrow \Omega\left(L^{n}[a, b]\right)$ число $\zeta=\rho_{m}(x)+i^{-1}-\left\|u_{m}-y\right\|_{L^{n}[a, b]}>0$. Так как функционал $\rho_{m}: H_{\text {со }} \rightarrow \mathbb{R}^{1}$ непрерывен, то найдется такой номер $j_{1}$, что при всех $j \geqslant j_{1}$ выполняется неравенство $\rho_{m}\left(x_{j}\right)+i^{-1}>\rho_{m}(x)+i^{-1}-2^{-1} \zeta$.

Покажем, что при всех $j \geqslant j_{1}$ выполняется вложение

$$
B_{L^{n}[a, b]}\left[y ; 2^{-1} \zeta\right] \subset B_{L^{n}[a, b]}\left[u_{m} ; \rho_{m}\left(x_{j}\right)+i^{-1}\right] .
$$

Действительно, пусть $v \in B_{L^{n}[a, b]}\left[y ; 2^{-1} \zeta\right]$. Тогда при всех $j \geqslant j_{1}$ получаем оценку

$$
\begin{aligned}
\left\|u_{m}-v\right\|_{L^{n}[a, b]} & \leqslant\left\|u_{m}-y\right\|_{L^{n}[a, b]}+\|y-v\|_{L^{n}[a, b]} \\
& \leqslant \rho_{m}(x)+i^{-1}-\zeta+2^{-1} \zeta<\rho_{m}\left(x_{j}\right)+i^{-1}-2^{-1} \zeta .
\end{aligned}
$$

Таким образом, вложение (68) справедливо при всех $j \geqslant j_{1}$. Далее, так как отображение со $\Phi: C^{n}[a, b] \rightarrow \Omega\left(\Pi\left[L^{n}[a, b]\right]\right)$ непрерывно, то для $y \in(\operatorname{co} \Phi)(x)$ найдется последовательность $y_{j} \in(\operatorname{co} \Phi)\left(x_{j}\right), j=1,2, \ldots$, такая, что $y_{j} \rightarrow y$ в пространстве $L^{n}[a, b]$ при $j \rightarrow \infty$. Отсюда вытекает, что найдется номер $j_{2}$ такой, что при всех $j \geqslant j_{2}$ выполняется включение $y_{j} \in B_{L^{n}[a, b]}\left[y ; 2^{-1} \zeta\right]$. Поэтому в силу соотношения (68), а также определения отображения $\Phi_{m i}: H_{\text {со }} \rightarrow \Omega\left(L^{n}[a, b]\right)$ получаем, что при всех $j \geqslant \max \left\{j_{1}, j_{2}\right\}$ вьполняется включение $y_{j} \in \Phi_{m i}\left(x_{j}\right)$. Таким образом, отображение $\Phi_{m i}: H_{\text {со }} \rightarrow \Omega\left(L^{n}[a, b]\right)$ полунепрерывно снизу. Отсюда вытекает, что отображения $\bar{\Phi}_{m i}: H_{\text {со }} \rightarrow \Omega\left(L^{n}[a, b]\right), m, i=1,2, \ldots$, определенные равенствами

$$
\bar{\Phi}_{m i}(x)=\overline{(\operatorname{co} \Phi)(x) \cap B_{L^{n}[a, b]}\left[u_{m} ; \rho_{m}(x)+i^{-1}\right]},
$$

также полунепрерывны снизу. Следовательно, согласно теореме Майкла [7] у отображения $\Phi_{m i}: H_{\text {со }} \rightarrow \Omega\left(L^{n}[a, b]\right)$ сушествует непрерывная ветвь $g_{m i}: H_{\mathrm{co}} \rightarrow$ $L^{n}[a, b], m, i=1,2, \ldots$.

Покажем, что последовательность $\left\{g_{m i}(x)\right\}$ плотна в $($ со $\Phi)(x)$ при любом $x \in H_{\text {со }}$. Действительно, пусть $\varepsilon>0, x \in H_{\text {со }}, u \in(\operatorname{co} \Phi)(x)$ и выберем такие $m, i=1,2, \ldots$, что выполняются неравенства

$$
\rho_{m}(x) \leqslant\left\|u_{m}-u\right\|_{L^{n}[a, b]} \leqslant i^{-1} \leqslant \frac{\varepsilon}{4} .
$$

Тогда $u \in B_{L^{n}[a, b]}\left[u_{m} ; \rho_{m}(x)+i^{-1}\right]$, причем согласно выбору чисел $m, i$ выполняется оценка $\rho_{m}(x)+i^{-1} \leqslant \varepsilon / 2$ и, следовательно, $\left\|u-g_{m i}(x)\right\|_{L^{n}[a, b]}<\varepsilon$. Таким образом, последовательность $\left\{g_{m i}(x)\right\}$ плотна в $(\operatorname{co} \Phi)(x)$ при любом $x \in H_{\text {со }}$.

Далее последовательность $\left\{g_{m i}\right\}$ будем обозначать через $\left\{g_{m}\right\}$. Определим для любого $m=1,2, \ldots$ функционалы $\eta_{m}: H_{\text {со }} \rightarrow \mathbb{R}^{1}, W_{m}: H_{\text {со }} \rightarrow \mathbb{R}^{1}$ равенствами

$$
\eta_{m}(x)=\left\|V^{-1}(x-\mathscr{P}(x))-g_{m}(x)\right\|_{L^{n}[a, b]}, \quad W_{m}(x)=W\left(x, g_{m}(x)\right),
$$


где $V^{-1}(y)$ - прообраз элемента $y \in C^{n}[a, b]$ (оператор $V: C^{n}[a, b] \rightarrow C^{n}[a, b]$ определен равенством (8)), отображение $\mathscr{P}: H_{\text {со }} \rightarrow C^{n}[a, b]$ задано соотношением (54), а функционал $W: H_{\text {со }} \rightarrow \mathbb{R}^{1}$ имеет вид (62).

Определим для любых $m, i=1,2, \ldots$ множества $H_{m i}^{1}, H_{m}, H_{m i}$, содержащиеся в $H_{\text {со }}$, следующим образом:

$$
\begin{gathered}
H_{m i}^{1}=\left\{x \in H_{\mathrm{co}}: W_{m}(x) \geqslant i^{-1}\right\}, \quad H_{m}=\left\{x \in H_{\mathrm{co}}: \eta_{m}(x) \geqslant 2^{-1} W_{m}(x)\right\}, \\
H_{m i}=H_{m i}^{1} \cap H_{m} .
\end{gathered}
$$

Покажем, что множества $H_{m i}$ замкнуты для любых $m, i=1,2, \ldots$ Прежде всего отметим, что согласно лемме 9 функционалы $W_{m}: H_{\text {со }} \rightarrow \mathbb{R}^{1}$ полунепрерывны сверху, и поэтому множества $H_{m i}^{1}$ замкнуты при любых $m, i=1,2, \ldots$.

Теперь покажем, что множество $H_{m}$ замкнуто для любого $m=1,2, \ldots$ Отметим, что согласно леммам 7-9 функционал $\eta_{m}: H_{\text {со }} \rightarrow \mathbb{R}^{1}$ полунепрерывен снизу, а функционал $W_{m}: H_{\text {со }} \rightarrow \mathbb{R}^{1}$ полунепрерывен сверху. Далее, пусть последо-

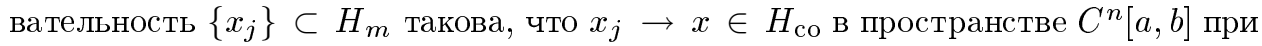
$j \rightarrow \infty$. Тогда получаем соотношения

$$
\eta_{m}(x) \leqslant \lim _{j \rightarrow \infty} \inf \eta_{m}\left(x_{j}\right) \leqslant \lim _{j \rightarrow \infty} 2^{-1} \sup W_{m}\left(x_{j}\right) \leqslant 2^{-1} W_{m}(x) .
$$

Следовательно, $x \in H_{m}$ (см. (69)) и множество $H_{m}$ замкнуто. Таким образом, замкнуто и множество $H_{m i}$ при любых $m, i=1,2, \ldots$ (см. (70)).

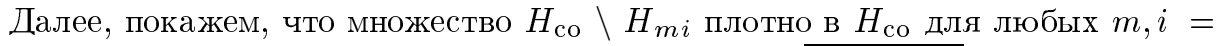

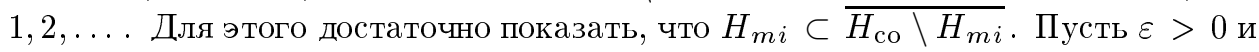
$x \in H_{m i}$. Покажем, что найдется такой $y \in H_{\mathrm{co}} \backslash H_{m i}$, для которого вьполняется неравенство $\|x-y\|_{C^{n}[a, b]}<\varepsilon$.

Так как $x \in H_{\text {со }}$, то согласно следствию $3 x$ - квазирешение включения (51). Это означает, что для $x \in H_{m i}$ найдутся элемент $p \in \Psi(x)$ и последовательность функций $z_{j} \in(\operatorname{ext} \Phi)(x), j=1,2, \ldots$, такие, что $y_{j}=p+V z_{j} \rightarrow x$ в пространстве $C^{n}[a, b]$ при $j \rightarrow \infty$. Тогда согласно свойству $\mathrm{B}^{*}$ из соотношений $(50),(12)$ для любого $j=1,2, \ldots$ и любого измеримого множества $\mathscr{U} \subset[a, b]$ получаем оценки

$$
\rho_{L^{n}(\mathscr{U})}\left[z_{j} ;(\operatorname{co} \Phi)\left(y_{j}\right)\right] \leqslant h_{L^{n}(\mathscr{U})}\left[(\operatorname{co} \Phi)(x) ;(\operatorname{co} \Phi)\left(y_{j}\right)\right] \leqslant \int_{\mathscr{U}}\left(\Gamma\left(Z\left(x-y_{j}\right)\right)\right)(s) d s,
$$

где отображение $Z: C^{n}[a, b] \rightarrow C_{+}^{1}[a, b]$ определено равенством (16).

Далее, пусть для любого $j=1,2, \ldots r_{j} \in \Psi\left(y_{j}\right)$ и удовлетворяет равенству

$$
\left\|p-r_{j}\right\|_{C^{n}[a, b]}=\rho_{C^{n}[a, b]}\left[p ; \Psi\left(y_{j}\right)\right] .
$$

Из непрерывности отображения $\Psi: C^{n}[a, b] \rightarrow \operatorname{comp}\left[C^{n}[a, b]\right]$ следует, что $r_{j} \rightarrow p$ в пространстве $C^{n}[a, b]$ при $j \rightarrow \infty$.

Пусть непрерьвная функция $\widetilde{\nu}_{j}:[a, b] \rightarrow[0, \infty), j=1,2, \ldots$, имеет вид:

$$
\widetilde{\nu}_{j}(t)=\int_{a}^{b}|V(t, s)| \Gamma\left(Z\left(x-y_{j}\right)\right)(s) d s+\left|p(t)-r_{j}(t)\right| .
$$


В силу того что $y_{j} \rightarrow x$ в пространстве $C^{n}[a, b]$ при $j \rightarrow \infty, \widetilde{\nu}_{j} \rightarrow 0$ в $C^{1}[a, b]$ при $j \rightarrow \infty$. Далее, согласно свойству В* и теореме 1 для каждой тройки функций $\left(y_{j}, r_{j}, z_{j}\right), j=1,2, \ldots$, найдется такое решение $x_{j} \in H_{\mathrm{co}}\left(x_{j}=\mathscr{P}\left(x_{j}\right)+V \widetilde{z}_{j}\right.$, $\mathscr{P}\left(x_{j}\right) \in \Psi\left(x_{j}\right), \widetilde{z}_{j} \in(\operatorname{co} \Phi)\left(x_{j}\right)$, где отображение $\mathscr{P}: H_{\text {со }} \rightarrow C^{n}[a, b]$ определено равенством (54)), которое обладает свойством: при любом $t \in[a, b]$ выполняется неравенство

$$
\left|x_{j}(t)-y_{j}(t)\right| \leqslant \xi_{j}(t)
$$

и при почти всех $t \in[a, b]$ справедлива оценка

$$
\left|z_{j}(t)-\widetilde{z}_{j}(t)\right| \leqslant\left(\Gamma Z\left(x-y_{j}\right)\right)(t)+\left(\Gamma \xi_{j}\right)(t),
$$

где $\xi_{j}$ - сумма ряда $(17)$ с функцией $\nu=\widetilde{\nu}_{j}$, заданной равенством (72). Из свойства $\mathrm{B}^{*}$ вытекает, что $\xi_{j} \rightarrow 0$ в пространстве $C^{1}[a, b]$ при $j \rightarrow \infty$. Следовательно, согласно оценке (74) получаем $z_{j}-\widetilde{z}_{j} \rightarrow 0$ в пространстве $L^{n}[a, b]$ при $j \rightarrow \infty$. Так как $z_{j} \in(\operatorname{ext} \Phi)(x)$ при $j=1,2, \ldots$, то для каждого $j=1,2, \ldots$ справедливо неравенство

$$
\rho_{L^{n}[a, b]}\left[\widetilde{z}_{j} ;(\operatorname{ext} \Phi)(x)\right] \leqslant\left\|\widetilde{z}_{j}-z_{j}\right\|_{L^{n}[a, b]} .
$$

Из последней оценки вытекает, что

$$
\lim _{j \rightarrow \infty} \rho_{L^{n}[a, b]}\left[\widetilde{z}_{j} ;(\operatorname{ext} \Phi)(x)\right]=0 .
$$

Так как $x \in H_{m i}$, то $W_{m}(x)>0$ (см. (62), (69), (70)). Отсюда и согласно равенству (75) найдутся такие числа $\delta>0$ и $j_{3}$, что при всех $j \geqslant j_{3}$ справедливо соотношение

$$
\rho_{L^{n}[a, b]}\left[\widetilde{z}_{j} ;(\operatorname{ext} \Phi)(x)\right]<2^{-1} W_{m}(x)-\delta .
$$

Поэтому при всех $j \geqslant j_{3}$ получаем неравенства

$$
\begin{aligned}
W_{m}(x) & \leqslant\left\|\widetilde{z}_{j}-g_{m}(x)\right\|_{L^{n}[a, b]}+\rho_{L^{n}[a, b]}\left[\widetilde{z}_{j} ;(\operatorname{ext} \Phi)(x)\right] \\
& <\left\|\widetilde{z}_{j}-g_{m}(x)\right\|_{L^{n}[a, b]}+2^{-1} W_{m}(x)-\delta .
\end{aligned}
$$

Таким образом, при всех $j \geqslant j_{3}$ имеем оценку

$$
\left\|\widetilde{z}_{j}-g_{m}(x)\right\|_{L^{n}[a, b]}>2^{-1} W_{m}(x)+\delta .
$$

Следовательно, при всех $j \geqslant j_{3}$ справедливо соотношение

$$
\widetilde{z}_{j} \notin \overline{B_{L^{n}[a, b]}\left[g_{m}(x) ; 2^{-1} W_{m}(x)+\delta\right]} .
$$

В силу непрерьвности отображения $g_{m}: H_{\text {со }} \rightarrow L^{n}[a, b]$ и полунепрерывности сверху функционала $W_{m}: C^{n}[a, b] \rightarrow \mathbb{R}^{1}$ (см. лемму 9) существует такое число $j_{4}$, что при всех $j \geqslant j_{4}$ выполняется вложение

$$
\overline{B_{L^{n}[a, b]}\left[g_{m}\left(x_{j}\right) ; 2^{-1} W_{m}\left(x_{j}\right)\right]} \subset \overline{B_{L^{n}[a, b]}\left[g_{m}(x) ; 2^{-1} W_{m}(x)+\delta\right]} .
$$


Поэтому из (76), (77) при всех $j \geqslant \max \left\{j_{3}, j_{4}\right\}$ получаем соотношение

$$
\widetilde{z}_{j} \notin \overline{B_{L^{n}[a, b]}\left[g_{m}\left(x_{j}\right) ; 2^{-1} W_{m}\left(x_{j}\right)\right]},
$$

т.е. при всех $j \geqslant \max \left\{j_{3}, j_{4}\right\}$ выполняется оценка $\eta_{m}\left(x_{j}\right)>2^{-1} W_{m}\left(x_{j}\right)$. Это означает, что $x_{j} \notin H_{m}$ (см. (69), (70)), а следовательно, $x_{j} \notin H_{m i}$ при всех $j \geqslant \max \left\{j_{3}, j_{4}\right\}$. Так как $\xi_{j} \rightarrow 0$ в $C^{1}[a, b]$, а $y_{j} \rightarrow x$ в $C^{n}[a, b]$ при $j \rightarrow \infty$, то из неравенства (73) вытекает, что $x_{j} \rightarrow x$ в пространстве $C^{n}[a, b]$ при $j \rightarrow \infty$. Поэтому в качестве $y \notin H_{m i}$ можно взять такой элемент $x_{\bar{j}}, \bar{j} \geqslant \max \left\{j_{3}, j_{4}\right\}$, для которого вьполняется неравенство $\left\|x-x_{\bar{j}}\right\|_{C^{n}[a, b]}<\varepsilon$. Таким образом, $H_{m i} \subset \overline{H_{\text {со } \backslash} \backslash H_{m i}}$ и множество $H_{\text {со }} \backslash H_{m i}$ плотно в $H_{\text {со }}$.

Далее, покажем, что справедливо вложение

$$
\bigcap_{m, i=1}^{\infty}\left[H_{\mathrm{co}} \backslash H_{m i}\right] \subset H_{\mathrm{ext}} .
$$

Действительно, пусть $x \in H_{\text {со }} \backslash H_{\mathrm{ext}}$ и

$$
x=\mathscr{P}(x)+V z, \quad \mathscr{P}(x) \in \Psi(x), \quad z \in(\operatorname{co} \Phi)(x),
$$

где отображение $\mathscr{P}: H_{\mathrm{co}} \rightarrow C^{n}[a, b]$ определено равенством $(54)$. Тогда $z \notin(\operatorname{ext} \Phi)(x)$ и, следовательно, $\rho_{L^{n}[a, b]}[z ;(\operatorname{ext} \Phi)(x)] \equiv \gamma>0$. Покажем, что $x \in H_{m i}$ при $i^{-1} \leqslant 2 \gamma / 3$ и при таком $m$, при котором выполняется неравенство

$$
\eta_{m}(x)<\frac{\gamma}{3}
$$

где $\eta_{m}(x)=\left\|z-g_{m}(x)\right\|_{L^{n}[a, b]}\left(z=V^{-1}(x-\mathscr{P}(x))\right)$. Такой номер $m$ сушествует, поскольку по построению последовательность $\left\{g_{m}(x)\right\}$ плотна в $($ со $\Phi)(x)$. Согласно определению функционала $W_{m}: C^{n}[a, b] \rightarrow \mathbb{R}^{1}$ (см. (62)) и неравенству (79) получаем оценку

$$
\gamma \leqslant\left\|g_{m}(x)-z\right\|_{L^{n}[a, b]}+W_{m}(x)<\frac{\gamma}{3}+W_{m}(x) .
$$

Отсюда получаем неравенство

$$
W_{m}(x)>\frac{2}{3} \gamma
$$

Из оценок (79), (80) вытекает соотношение

$$
\eta_{m}(x)<2^{-1} W_{m}(x)
$$

Это означает, что $x \in H_{m}$ (см. (69)). Кроме того, так как $i^{-1} \leqslant 2 \gamma / 3$, то из (80) следует оценка $W_{m}(x)>i^{-1}$, т.е. $x \in H_{m i}^{1}$ (см. (69)), и поэтому $x \in H_{m i}$ (см. (70)). Таким образом,

$$
x \notin \bigcap_{m, i=1}^{\infty}\left[H_{\mathrm{co}} \backslash H_{m i}\right]
$$

и поэтому вложение (78) справедливо.

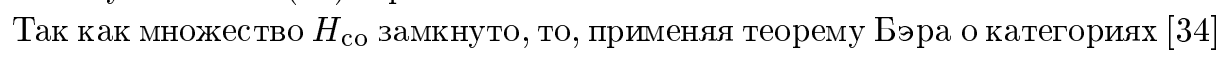

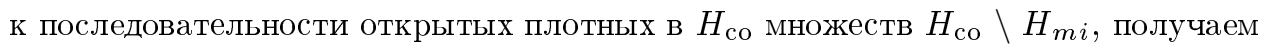
равенство

$$
\overline{\bigcap_{m, i=1}^{\infty}\left[H_{\mathrm{co}} \backslash H_{m i}\right]}=H_{\mathrm{co}} .
$$

Из соотношений $(78),(81)$ вытекает, что $H_{\text {со }}=\overline{H_{\text {ext }}}$. Теорема доказана. 
ЗАмечание 14. Отметим, что теорема 6 уточняет результаты работ [3], [5], [6], поскольку она не предполагает, что отображение $\Psi: C^{n}[a, b] \rightarrow \operatorname{comp}\left[C^{n}[a, b]\right]$ имеет выпуклые образы.

\section{$\S 2$. Возмущенное включение с внешними и внутренними возмушениями}

Приближенные решения часто используются при исследовании дифференциальных уравнений и включений. Для дифференциальных уравнений с кусочно непрерывной правой частью в [35] понятие приближенного решения учитывало не только малые изменения правой части в областях непрерывности, но и малые изменения границ этих областей. И это приближенное решение в [35] определялось как решение дифференциального уравнения с внутренними и внешними возмущениями, причем нормы значений возмущений не превосходили малого параметра $\delta>0$. Для дифференциальных включений обшее определение приближенного решения ( $\delta$-решения) было впервые введено А. $\Phi$. Филипповым (см. [14]). Это определение решения является более общим понятием приближенного решения даже для дифференциальных уравнений. Отметим, что данное А.. . Филипповым определение решения для дифференциальных включений с вьпуклозначной и полунепрерывной сверху правой частью обладает фундаментальным свойством: пределы сходящихся последовательностей приближенных решений являются решениями (см. [14]). Поэтому это определение играет важную роль при исследовании дифференциальных уравнений с разрывной правой частью и дифференциальных включений. Для изучения аппроксимаций дифференциальных включений с невыпуклой правой частью в работах [16], [31]-[33], [36]-[38] предложено несколько другое определение понятия приближенного решения. Отличие сформулированного в работах [16], [31]-[33], [36]-[38] решения от приближенного решения по Филиппову заключается в том, что значения многозначных отображений, определяющие "приближенные дифференциальные включения", не "овыпукляются". Как показано в [16], [31]-[33], [36]-[38], такое определение приближенного решения полезно для исследования аппроксимаций дифференциальных включений с невьпуклой правой частью. Оно, прежде всего, позволило доказать, что аппроксимация включений с невыпуклой правой частью может быть неустойчивой операцией, поскольку "небольшие" изменения правой части могут привести к существенному изменению множества решений. Кроме того, это определение позволяет получить необходимое и достаточное условие, когда аппроксимация является устойчивой (в работе [16] это условие названо принципом плотности).

Результаты, доказанные в этом параграфе, используют понятие приближенного решения, которое аналогично предложенному в работах [16], [31]-[33], [36]-[38], и примыкают к этим исследованиям.

2.1. Возмущенное включение с внешними возмущениями. Здесь рассматривается включение (7) с внешними возмушениями. Такие возмушения в приложениях имеют место, поскольку они характеризуют погрешность вычислений значений соответствуюших многозначных отображений. Как доказывается здесь, этими возмущениями нельзя пренебрегать, так как они могут вызвать значительные изменения множества решений возмушенного включения, если отображение 
$\Phi: C^{n}[a, b] \rightarrow \Pi\left[L^{n}[a, b]\right]$ не обладает свойством выпуклости значений, т.е. когда отображение, порожденное правой частью включения (7), не обладает свойством замкнутости в пространстве $C^{n}[a, b]$ образов.

Далее будем предполагать, что отображение $\Psi: C^{n}[a, b] \rightarrow \operatorname{comp}\left[C^{n}[a, b]\right]$ непрерывно по Хаусдорфу.

Обозначим через $K([a, b] \times[0, \infty))$ множество всех функций $\eta:[a, b] \times[0, \infty) \rightarrow$ $[0, \infty)$, обладающих следуюшими свойствами: при каждом $\delta \geqslant 0$ функция $\eta(\cdot, \delta) \in$ $L^{1}[a, b] ;$ для каждого $\delta \geqslant 0$ найдется такая функция $\beta_{\delta}(\cdot) \in L^{1}[a, b]$, что при почти всех $t \in[a, b]$ и всех $\tau \in[0, \delta]$ вьполняется неравенство $\eta(t, \tau) \leqslant \beta_{\delta}(t)$, при почти всех $t \in[a, b]$ справедливы равенства $\lim _{\delta \rightarrow 0+0} \eta(t, \delta)=\eta(t, 0)=0$.

Обозначим через $P\left(C^{n}[a, b] \times[0, \infty)\right)$ множество всех непрерывных функций $\omega: C^{n}[a, b] \times[0, \infty) \rightarrow[0, \infty)$, для которых для любого $x \in C^{n}[a, b]$ справедливо соотношение $\omega(x, 0)=0$ и для любых $(x, \delta) \in C^{n}[a, b] \times(0, \infty)$ выполняется неравенство $\omega(x, \delta)>0$.

Рассмотрим отображение $\Delta:[a, b] \times C^{n}[a, b] \rightarrow \operatorname{comp}\left[\mathbb{R}^{n}\right]$, порождающее отображение $\Phi: C^{n}[a, b] \rightarrow \Pi\left[L^{n}[a, b]\right]$. Будем предполагать, что при почти всех $t \in[a, b]$ отображение $\Delta(t, \cdot)$ непрерывно на $C^{n}[a, b]$.

Как было отмечено выше, для оператора Немыцкого $\mathscr{N}: C^{n}[a, b] \rightarrow \Pi\left[L^{n}[a, b]\right]$, порожденного функцией $F:[a, b] \times \mathbb{R}^{n} \rightarrow \operatorname{comp}\left[\mathbb{R}^{n}\right]$, удовлетворяющей условиям Каратеодори, отображение $\Delta_{1}:[a, b] \times C^{n}[a, b] \rightarrow \operatorname{comp}\left[\mathbb{R}^{n}\right]$ имеет вид $\Delta_{1}(t, x)=$ $F(t, x(t))$. Поэтому отображение $\Delta_{1}(\cdot, \cdot)$ при почти всех $t \in[a, b]$ непрерьвно на $C^{n}[a, b]$.

Для произведения $\mathscr{N} \Theta: C^{n}[a, b] \rightarrow \Pi\left[L^{n}[a, b]\right]$ порождающее отображение $\Delta_{2}:$ $[a, b] \times C^{n}[a, b] \rightarrow \operatorname{comp}\left[\mathbb{R}^{n}\right]$ имеет вид

$$
\Delta_{2}(t, x)=F(t,(\Theta x)(t))
$$

которое при почти всех $t \in[a, b]$ непрерывно на $C^{n}[a, b]$ (непрерывный оператор $\Theta: C^{n}[a, b] \rightarrow L^{n}[a, b]$ задан соотношением (30)). Таким образом, класс отображений $\Phi: C^{n}[a, b] \rightarrow \Pi\left[L^{n}[a, b]\right]$, для которых порождающие их отображения $\Delta:[a, b] \times C^{n}[a, b] \rightarrow \operatorname{comp}\left[\mathbb{R}^{n}\right]$ при почти всех $t \in[a, b]$ непрерывны на $C^{n}[a, b]$, шиирок.

Рассмотрим оператор $\Phi: C^{n}[a, b] \rightarrow \Pi\left[L^{n}[a, b]\right]$ и порождающее его отображение $\Delta:[a, b] \times C^{n}[a, b] \rightarrow \operatorname{comp}\left[\mathbb{R}^{n}\right]$. Значения отображения $\Delta(\cdot, \cdot)$, а следовательно, и образы оператора $\Phi(\cdot)$ могут вычисляться с некоторой степенью точности, которую можно задать функцией $\eta(\cdot, \cdot) \in K([a, b] \times[0, \infty))$. В связи с этим рассмотрим отображение $\Delta_{\eta}:[a, b] \times C^{n}[a, b] \times[0, \infty) \rightarrow \operatorname{comp}\left[\mathbb{R}^{n}\right]$, заданное равенством

$$
\Delta_{\eta}(t, x, \delta)=(\Delta(t, x))^{\eta(t, \delta)},
$$

где функция $\eta(\cdot, \cdot) \in K([a, b] \times[0, \infty))$ в каждой точке $(t, x) \in[a, b] \times C^{n}[a, b]$ при каждом фиксированном $\delta \in[0, \infty)$ определяет погрешность вычисления значения порождаюшего отображения $\Delta(\cdot, \cdot)$, причем эти погрешности равномерны относительно переменной $x \in C^{n}[a, b]$. Далее функцию $\eta(\cdot, \cdot)$ будем называть $p a-$ диусом внешних возмущений порохсдаюего отображсения $\Delta(\cdot, \cdot)$ или просто радиусом внешних возмущений. 
Заметим, что из $(82)$ при всех $(t, x) \in[a, b] \times C^{n}[a, b]$ вытекает равенство

$$
h\left[\Delta(t, x) ; \Delta_{\eta}(t, x, \delta)\right]=\eta(t, \delta)
$$

Таким образом, из соотношения (83) вытекает, что для каждой функции $\eta(\cdot, \cdot) \in$ $K([a, b] \times[0, \infty))$ при почти всех $t \in[a, b]$ и всех $x \in C^{n}[a, b]$ справедливо соотношение

$$
\lim _{\delta \rightarrow 0+0} h\left[\Delta(t, x) ; \Delta_{\eta}(t, x, \delta)\right]=0 .
$$

Поэтому все отображения $\Delta_{\eta}:[a, b] \times C^{n}[a, b] \times[0, \infty) \rightarrow \operatorname{comp}\left[\mathbb{R}^{n}\right]$, определенные равенством $(82)$ и зависящие от радиуса внешних возмушений $\eta(\cdot, \cdot) \in K([a, b] \times$ $[0, \infty))$, близки (в смысле равенства (84)) к отображению $\Delta:[a, b] \times C^{n}[a, b] \rightarrow$ $\operatorname{comp}\left[\mathbb{R}^{n}\right]$. Это приближение будем назьвать поточечной аппроксимацией вложсением порохсдаюего отображсеня $\Delta(\cdot, \cdot)$ или просто поточечной аппроксимачией вложсением. Отображение $\Delta_{\eta}:[a, b] \times C^{n}[a, b] \times[0, \infty) \rightarrow \operatorname{comp}\left[\mathbb{R}^{n}\right]$ будем называть аппроксимирующим оператором.

Далее, определим отображение $\Phi_{\eta}: C^{n}[a, b] \times[0, \infty) \rightarrow \Pi\left[L^{n}[a, b]\right]$, заданное соотношением

$$
\Phi_{\eta}(x, \delta)=S\left(\Delta_{\eta}(\cdot, x, \delta)\right)
$$

Из равенств (85), (83) для любого $x \in C^{n}[a, b]$ вытекает

$$
h_{L^{n}[a, b]}\left[\Phi_{\eta}(x, \delta) ; \Phi(x)\right]=\int_{a}^{b} \eta(t, \delta) d t .
$$

Поэтому из (86) и теоремы Лебега следует равенство

$$
\lim _{\delta \rightarrow 0+0} h_{L^{n}[a, b]}\left[\Phi_{\eta}(x, \delta) ; \Phi(x)\right]=0 .
$$

Таким образом, все отображения $\Phi_{\eta}: C^{n}[a, b] \times[0, \infty) \rightarrow \Pi\left[L^{n}[a, b]\right]$, заданные равенствами $(82),(85)$ и зависяшие от радиуса внешних возмушений $\eta(\cdot, \cdot) \in$ $K([a, b] \times[0, \infty))$, близки (в смысле равенства $(87))$ к отображению $\Phi: C^{n}[a, b] \rightarrow$ $\Pi\left[L^{n}[a, b]\right]$. Это приближение оператора $\Phi(\cdot)$ будем называть аппроксимаиией вложсением в среднем. Отображение $\Phi_{\eta}: C^{n}[a, b] \times[0, \infty) \rightarrow \Pi\left[L^{n}[a, b]\right]$ будем называть аппроксимирующим оператором. Таким образом, из поточечной аппроксимации отображения $\Delta(\cdot, \cdot)$, порождающего оператор $\Phi(\cdot)$, вытекает аппроксимация отображения $\Phi(\cdot)$ в среднем.

Лемма 10. Пусть $X$ - нормированное пространство, $U \subset X-$ выпуклое множество. Тогда для любых $x_{1}, x_{2} \in U$ и всех $r_{1}, r_{2}>0$ справедливо неравенство

$$
h_{X}\left[B_{X}\left[x_{1} ; r_{1}\right] \cap U ; B_{X}\left[x_{2} ; r_{2}\right] \cap U\right] \leqslant\left\|x_{1}-x_{2}\right\|_{X}+\left|r_{2}-r_{1}\right| .
$$


ДокАЗАТЕльство. Для доказательства леммы достаточно показать, что для любого $z \in B_{X}\left[x_{2} ; r_{2}\right] \cap U$ выполняется неравенство

$$
\rho_{X}\left[z ; B_{X}\left[x_{1} ; r_{1}\right] \cap U\right] \leqslant\left\|x_{1}-x_{2}\right\|_{X}+\left|r_{1}-r_{2}\right| .
$$

Докажем это неравенство. Если $\rho_{X}\left[z ; B_{X}\left[x_{1} ; r_{1}\right] \cap U\right]=0$, то неравенство справедливо. Поэтому предположим, что $z \notin \overline{B_{X}\left[x_{1} ; r_{1}\right] \cap U}$. Это означает, что $z \notin \overline{B_{X}\left[x_{1} ; r_{1}\right]}$, т.е. $\left\|z-x_{1}\right\|_{X}>r_{1}$. Обозначим $\sigma(\lambda)=\lambda z+(1-\lambda) x_{1}, \lambda \in[0,1]$. Так как $z, x_{1} \in U$, а $U$ - выпуклое множество, то $\sigma(\lambda) \in U$ для любого $\lambda \in[0,1]$. Рассмотрим функцию $\psi:[0,1] \rightarrow \mathbb{R}$, определенную равенством

$$
\psi(\lambda)=\left\|x_{1}-\sigma(\lambda)\right\|_{X}
$$

Из определения функции $\psi(\cdot$ ) следует

$$
\psi(\lambda)=\left\|\lambda x_{1}+(1-\lambda) x_{1}-\lambda z-(1-\lambda) x_{1}\right\|_{X}=\lambda\left\|x_{1}-z\right\|_{X},
$$

из чего вытекает $\psi(0)=0, \psi(1)=\left\|x_{1}-z\right\|_{X}>r_{1}$. Так как функция $\psi(\cdot)$ непрерывна на $[0,1]$, то существует такое число $\lambda_{0} \in(0,1)$, что $\psi\left(\lambda_{0}\right)=r_{1}$. Это означает, что элемент $\sigma\left(\lambda_{0}\right)=\lambda_{0} z+\left(1-\lambda_{0}\right) x_{1}$ удовлетворяет равенству $\left\|x_{1}-\sigma\left(\lambda_{0}\right)\right\|_{X}=r_{1}$. Рассмотрим

$$
\begin{aligned}
\left\|z-\sigma\left(\lambda_{0}\right)\right\|_{X} & =\left\|\lambda_{0} z+\left(1-\lambda_{0}\right) z-\lambda_{0} z-\left(1-\lambda_{0}\right) x_{1}\right\|_{X}=\left(1-\lambda_{0}\right)\left\|z-x_{1}\right\|_{X} \\
& =\left\|z-x_{1}\right\|_{X}-\lambda_{0}\left\|z-x_{1}\right\|_{X}=\left\|z-x_{1}\right\|_{X}-r_{1} .
\end{aligned}
$$

Поэтому получаем

$$
\begin{aligned}
& \rho_{X}\left[z ; B_{X}\left[x_{1} ; r_{1}\right] \cap U\right] \leqslant\left\|z-\sigma\left(\lambda_{0}\right)\right\|_{X}=\left\|z-x_{1}\right\|_{X}-r_{1} \\
& \quad \leqslant\left\|z-x_{2}\right\|_{X}+\left\|x_{2}-x_{1}\right\|_{X}-r_{1} \leqslant\left\|x_{2}-x_{1}\right\|_{X}+r_{2}-r_{1} \\
& \quad \leqslant\left\|x_{2}-x_{1}\right\|_{X}+\left|r_{2}-r_{1}\right| .
\end{aligned}
$$

Лемма доказана.

СлеДСТвИЕ 4. Пусть X - нормированное пространство. Тогда для любъх $x_{1}, x_{2} \in X$ и всех $r_{1}, r_{2}>0$ справедливо равенство

$$
h_{X}\left[B_{X}\left[x_{1} ; r_{1}\right] ; B_{X}\left[x_{2} ; r_{2}\right]\right]=\left\|x_{2}-x_{1}\right\|_{X}+\left|r_{2}-r_{1}\right| .
$$

ЗАмечАниЕ 15 . Очевидно, что соотношения (88), (89) остаются справедливыми и в случае, когда в левых частях этих неравенств будут стоять различные замькания множеств.

Пусть $U \subset C^{n}[a, b]$ - выпуклое замкнутое множество, и пусть

$$
\omega(\cdot, \cdot) \in P\left(C^{n}[a, b] \times[0, \infty)\right) .
$$

Рассмотрим многозначное отображение $M_{U}(\omega): U \times[0, \infty) \rightarrow \Omega(U)$, имеющее вид

$$
M_{U}(\omega)(x, \delta)=\overline{B_{C^{n}[a, b]}[x ; \omega(x, \delta)]} \cap U .
$$

Из неравенства (88) вытекает 
Лемма 11. Пусть $U \subset C^{n}[a, b]$ - выпуклое замкнутое множсество, и пусть $\omega(\cdot, \cdot) \in P\left(C^{n}[a, b] \times[0, \infty)\right)$. Тогда многозначное отобрахсение $M_{U}(\omega): U \times$ $[0, \infty) \rightarrow \Omega(U)$, заданное соотношением $(90)$, непрерывно по Хаусдорфу.

Определим отображение $\varphi_{U}(\omega):[a, b] \times U \times[0, \infty) \rightarrow[0, \infty)$ соотношением

$$
\varphi_{U}(\omega)(t, x, \delta)=\sup _{y \in M_{U}(\omega)(x, \delta)} h[\Delta(t, x) ; \Delta(t, y)]
$$

где отображение $M_{U}(\omega): U \times[0, \infty) \rightarrow \Omega(U)$ задано равенством $(90)$, а

$$
\Delta:[a, b] \times C^{n}[a, b] \rightarrow \operatorname{comp}\left[\mathbb{R}^{n}\right]
$$

- отображение, порождающее оператор $\Phi: C^{n}[a, b] \rightarrow \Pi\left[L^{n}[a, b]\right]$.

Значение функции $\varphi_{U}(\omega)(\cdot, \cdot, \cdot)$ в точке $(t, x, \delta) \in[a, b] \times U \times[0, \infty)$, на наш взгляд, естественно назвать модулем непрерьвности отображсения $\Delta:[a, b] \times$ $C^{n}[a, b] \rightarrow \operatorname{comp}\left[\mathbb{R}^{n}\right]$ в точке $(t, x)$ по переменной $x$ на множестве $U$, функцию $\omega(\cdot, \cdot)$ - функцией радиуса модуля непрерьвности отображения или просто

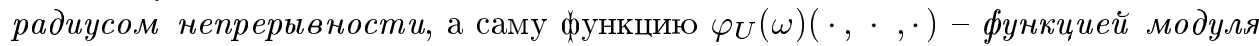
непрерывности или просто модулем непрерывности отображсения $\Delta:[a, b] \times$ $C^{n}[a, b] \rightarrow \operatorname{comp}\left[\mathbb{R}^{n}\right]$ на множестве $U$ относительно радиуса непрерывности $\omega(\cdot, \cdot)$.

ЛЕмма 12. Пусть $U$ - непустое выпуклое компактное множество пространства $C^{n}[a, b]$, и пусть $\omega(\cdot, \cdot) \in P\left(C^{n}[a, b] \times[0, \infty)\right)$. Далее, пусть отображсение $\Delta:[a, b] \times C^{n}[a, b] \rightarrow \operatorname{comp}\left[\mathbb{R}^{n}\right]$, порождающее оператор $\Phi: C^{n}[a, b] \rightarrow$ $\Pi\left[L^{n}[a, b]\right]$, при почти всех $t \in[a, b]$ непрерьвно на $U$, и пусть существует такая суммируемая функиия $\beta_{U}:[a, b] \rightarrow[0, \infty)$, что при почти всех $t \in[a, b]$ и любых $x \in U$ справедливо неравенство $\|\Delta(t, x)\| \leqslant \beta_{U}(t)$. Тогда отображение $\varphi_{U}(\omega):[a, b] \times U \times[0, \infty) \rightarrow[0, \infty)$, заданное равенством $(91)$, обладает следующими свойствами:

а) для любьх $(x, \delta) \in U \times[0, \infty)$ функиия $\varphi_{U}(\omega)(\cdot, x, \delta)$ измерима;

b) при почти всех $t \in[a, b]$ отобрахсение $\varphi_{U}(t, \cdot, \cdot)$ непрерывно на $U \times$ $[0, \infty)$

c) для любого $x \in U$ при почти всех $t \in[a, b]$ выполняется соотношение

$$
\lim _{z \rightarrow x} \varphi_{U \rightarrow 0+0}(\omega)(t, z, \delta)=0
$$

d) существует такая суммируемая функиия $p_{U}:[a, b] \rightarrow[0, \infty)$, что при почти всех $t \in[a, b]$ для любого $x \in U$ и для любого $\delta \in[0, \infty)$ справедлива оценка $\varphi_{U}(\omega)(t, x, \delta) \leqslant p_{U}(t)$.

ДоказАТЕЛьство. Прежде всего, так как для почти всех $t \in[a, b]$ и для любых $x, y \in U$ имеет место неравенство

$$
h[\Delta(t, x) ; \Delta(t, y)] \leqslant\|\Delta(t, x)\|+\|\Delta(t, y)\| \leqslant 2 \beta_{U}(t)
$$


то суммируемая функция $p_{U}:[a, b] \rightarrow[0, \infty)$ определяется равенством $p_{U}(t)=$ $2 \beta_{U}(t)$.

Пусть, далее, $t \in[a, b]$ - точка, в которой отображение $\Delta(t, \cdot)$ непрерьвно на $C^{n}[a, b]$. Рассмотрим отображение $\varphi_{U}(\omega)(t, \cdot, \cdot)$. Покажем, что это отображение непрерывно по совокупности аргументов на $U \times[0, \infty)$. Действительно, пусть последовательности $x_{i}, \delta_{i}$ таковы, что $x_{i}(\in U) \rightarrow x$ в $C^{n}[a, b]$ и $\delta_{i}(\in[0, \infty)) \rightarrow$ $\delta(\in[0, \infty))$ при $i \rightarrow \infty$. Пусть $\varepsilon>0$, и пусть для любого $i=1,2, \ldots$ функции $y_{i} \in M_{U}(\omega)\left(x_{i}, \delta_{i}\right)$ удовлетворяют неравенству

$$
\varphi_{U}(\omega)\left(t, x_{i}, \delta_{i}\right) \leqslant h\left[\Delta(t, x) ; \Delta\left(t, y_{i}\right)\right]+\varepsilon
$$

Не уменьшая обшности, можно считать, что $y_{i} \rightarrow y$ в $C^{n}[a, b]$ при $i \rightarrow \infty$. В силу леммы $11 y \in M_{U}(\omega)(x, \delta)$. Отсюда согласно неравенствам (93) для любого $i=$ $1,2, \ldots$ получаем оценку

$$
\varphi_{U}(\omega)\left(t, x_{i}, \delta_{i}\right) \leqslant h\left[\Delta(t, y) ; \Delta\left(t, y_{i}\right)\right]+\varphi_{U}(\omega)(t, x, \delta)+\varepsilon
$$

Следовательно,

$$
\varlimsup_{i \rightarrow \infty} \varphi_{U}(\omega)\left(t, x_{i}, \delta_{i}\right) \leqslant \varphi_{U}(\omega)(t, x, \delta) .
$$

Теперь покажем, что функция $\varphi_{U}(\omega)(t, \cdot, \cdot)$ полунепрерьвна снизу. Действительно, пусть последовательности $\left\{x_{i}\right\}$ и $\left\{\delta_{i}\right\}$ выбраны как в предыдушем случае. И пусть функция $y \in M_{U}(\omega)(x, \delta)$ удовлетворяет равенству

$$
\varphi_{U}(\omega)(t, x, \delta) \leqslant h[\Delta(t, x) ; \Delta(t, y)]+\varepsilon
$$

Согласно лемме 11 для каждого $i=1,2, \ldots$ найдется функция $y_{i} \in M_{U}(\omega)\left(x_{i}, \delta_{i}\right)$ такая, что $y_{i} \rightarrow y$ в $C^{n}[a, b]$ при $i \rightarrow \infty$. Поэтому из (94) получаем оценку

$$
\varphi_{U}(\omega)(t, x, \delta) \leqslant h\left[\Delta(t, x) ; \Delta\left(t, x_{i}\right)\right]+h\left[\Delta\left(t, y_{i}\right) ; \Delta(t, y)\right]+\varphi_{U}(\omega)\left(t, x_{i}, \delta_{i}\right)+\varepsilon
$$

Переходя в последнем неравенстве к пределу, получаем соотношение

$$
\varphi_{U}(\omega)(t, x, \delta) \leqslant \lim _{i \rightarrow \infty} \varphi_{U}(\omega)\left(t, x_{i}, \delta_{i}\right)
$$

Таким образом, функция $\varphi_{U}(\omega)(t, \cdot, \cdot)$ непрерывна по совокупности аргументов.

Так как для любого $x \in U$ выполняется равенство $\varphi_{U}(\omega)(t, x, 0)=0$, то равенство (92) имеет место.

Пусть $Q \subset M_{U}(\omega)(x, \delta)$ - счетное всюду плотное множество в $M_{U}(\omega)(x, \delta)$. Тогда из измеримости функции $h[\Delta(\cdot, x) ; \Delta(\cdot, y)]:[a, b] \rightarrow[0, \infty)$ и из равенства

$$
\varphi_{U}(\omega)(t, x, \delta)=\sup _{y \in Q} h[\Delta(t, x) ; \Delta(t, y)]
$$

вытекает измеримость функции $\varphi_{U}(\omega)(\cdot, x, \delta)$. Лемма доказана. 
ОПРеДЕЛЕНИЕ. Пусть $U \subset C^{n}[a, b]$. Будем говорить, что функция $\eta(\cdot, \cdot) \in$ $K([a, b] \times[0, \infty))$ равномерно на множсестве $U \subset C^{n}[a, b]$ оченивает сверху относительно радиуса непрерывности $\omega(\cdot, \cdot) \in P\left(C^{n}[a, b] \times[0, \infty)\right)$ модуль непрерывности отображения $\Delta:[a, b] \times C^{n}[a, b] \rightarrow \operatorname{comp}\left[\mathbb{R}^{n}\right]$, порождающего оператор $\Phi: C^{n}[a, b] \rightarrow \Pi\left[L^{n}[a, b]\right]$, если для любого $\varepsilon>0$ сушествует такое $\delta(\varepsilon)>0$, что при почти всех $t \in[a, b]$ и всех $x \in U$ и $\delta \in(0, \delta(\varepsilon)]$ выполняется неравенство

$$
\varphi_{U}(\omega)(t, x, \delta) \leqslant \eta(t, \varepsilon),
$$

где отображение $\varphi_{U}:[a, b] \times U \times[0, \infty) \rightarrow[0, \infty)$ определено соотношением $(91)$.

Пусть $U \subset C^{n}[a, b]$, и пусть $\omega(\cdot, \cdot) \in P\left(C^{n}[a, b] \times[0, \infty)\right)$. Определим функцию $\lambda_{U}(\omega):[a, b] \times[0, \infty) \rightarrow[0, \infty)$ соотношением

$$
\lambda_{U}(\omega)(t, \delta)=\sup _{x \in U} \varphi_{U}(\omega)(t, x, \delta)
$$

Из леммы 12 вытекает

СлЕДСТВИЕ 5. Пусть $U$ - непустое выпуклое компактное множество пространства $C^{n}[a, b], \omega(\cdot, \cdot) \in P\left(C^{n}[a, b] \times[0, \infty)\right)$. Далее, пусть отображение $\Delta:[a, b] \times C^{n}[a, b] \rightarrow \operatorname{comp}\left[\mathbb{R}^{n}\right]$, порождающее оператор $\Phi: C^{n}[a, b] \rightarrow$ $\Pi\left[L^{n}[a, b]\right]$, при почти всех $t \in[a, b]$ непрерывно на $C^{n}[a, b]$, и пусть существует такая суммируемая функция $\beta_{U}:[a, b] \rightarrow[0, \infty)$, что при почти всех $t \in[a, b]$ и любых $x \in U$ справедливо неравенство $\|\Delta(t, x)\| \leqslant$ $\beta_{U}(t)$. Тогда функция $\lambda_{U}(\omega):[a, b] \times[0, \infty) \rightarrow[0, \infty)$, определенная равенством (96), принадлежит множеству $K([a, b] \times[0, \infty))$ и равномерно на множестве $U \subset C^{n}[a, b]$ оценивает сверху относительно радиуса непрерьвности $\omega(\cdot, \cdot) \in P\left(C^{n}[a, b] \times[0, \infty)\right)$ модуль непрерьвности отображения $\Delta(\cdot, \cdot)$.

ДоказАТЕЛЬСтво. Действительно, измеримость функции $\lambda_{U}(\omega)(\cdot, \cdot)$ по первому аргументу вытекает из равенства

$$
\lambda_{U}(\omega)(t, \delta)=\sup _{x \in Q} \varphi_{U}(\omega)(t, x, \delta)
$$

где $Q$ - счетное всюду плотное множество в $U$. Далее, в силу условия леммы и определения функции $\varphi_{U}(\omega)(\cdot, \cdot, \cdot)$ для любого $\delta \in[0, \infty)$ справедливо неравенство

$$
\lambda_{U}(\omega)(t, \delta) \leqslant 2 \beta_{U}(t)
$$

Следовательно, при каждом $\delta \in[0, \infty) \lambda_{U}(\omega)(\cdot, \delta) \in L^{1}[a, b]$. Покажем, что при почти всех $t \in[a, b]$ имеет место равенство

$$
\lim _{\delta \rightarrow 0+0} \lambda_{U}(\omega)(t, \delta)=0 .
$$

Пусть $t \in[a, b]$ - точка, в которой при любом $x \in U$ выполняется равенство

$$
\lim _{z \rightarrow x \delta \rightarrow 0+0} \varphi_{U}(\omega)(t, z, \delta)=0
$$


Допустим, что сушествуют такое $\varepsilon>0$ и такая последовательность $\delta_{i}>0$, $i=1,2, \ldots$, удовлетворяюшая условию $\delta_{i} \rightarrow 0$, что для любого $i=1,2, \ldots$ выполняется неравенство $\lambda_{U}(\omega)\left(t, \delta_{i}\right)>\varepsilon$. Тогда для каждого $i=1,2, \ldots$ существует $x_{i} \in U$, для которого справедлива оценка

$$
\varphi_{U}(\omega)\left(t, x_{i}, \delta_{i}\right)>\varepsilon
$$

Так как $U$ - компактное множество, то можно предположить, что $x_{i} \rightarrow x(\in U)$ в пространстве $C^{n}[a, b]$ при $i \rightarrow \infty$. Из неравенств (98) следует, что

$$
\lim _{i \rightarrow \infty} \varphi_{U}(\omega)\left(t, x_{i}, \delta_{i}\right) \geqslant \varepsilon>0,
$$

но это противоречит (97). Так как (97) выполняется при почти всех $t \in[a, b]$, то

$$
\lim _{\delta \rightarrow 0+0} \lambda_{U}(\omega)(t, \delta)=0
$$

справедливо почти всюду на $[a, b]$. Таким образом, функция $\lambda_{U}(\omega)(\cdot, \cdot) \in K([a, b] \times$ $[0, \infty))$.

Докажем теперь, что функция $\lambda_{U}(\omega)(\cdot, \cdot) \in K([a, b] \times[0, \infty))$ равномерно на множестве $U \subset C^{n}[a, b]$ оценивает сверху модуль непрерывности отображения $\Delta:[a, b] \times C^{n}[a, b] \rightarrow \operatorname{comp}\left[\mathbb{R}^{n}\right]$. Действительно, согласно равенству (96) для любых $x \in U, \varepsilon \in(0, \infty)$ и при почти всех $t \in[a, b]$ выполняется соотношение $\varphi_{U}(\omega)(t, x, \varepsilon) \leqslant \lambda_{U}(\omega)(t, \varepsilon)$. Так как $U \subset C^{n}[a, b]-$ компакт и $\omega(\cdot, \cdot) \in$ $P\left(C^{n}[a, b] \times[0, \infty)\right)$, то найдется такое число $\delta(\varepsilon)>0$, что для любых $x \in U$ и $\delta \in$ $(0, \delta(\varepsilon)]$ имеет место неравенство $\omega(x, \delta)<\omega(x, \varepsilon)$. Поэтому из равенств $(90),(91)$ для любых $x \in U, \delta \in(0, \delta(\varepsilon)]$ и при почти всех $t \in[a, b]$ вытекает соотношение $\varphi_{U}(\omega)(t, x, \delta) \leqslant \varphi_{U}(\omega)(t, x, \varepsilon)$. Теперь из приведенных выше оценок следует, что функция $\lambda_{U}(\omega)(\cdot, \cdot)$ равномерно на множестве $U$ оценивает сверху модуль непрерывности отображения $\Delta(\cdot, \cdot)$. Следствие доказано.

ЗАмЕчАнИЕ 16 . Следствие 5 устанавливает, что если $U$ - непустое выпуклое компактное множество пространства $C^{n}[a, b]$, то найдется хотя бы одна функция $\eta(\cdot, \cdot) \in K([a, b] \times[0, \infty))$, которая равномерно на множестве $U$ оценивает сверху относительно фиксированного радиуса непрерывности $\omega(\cdot, \cdot) \in P\left(C^{n}[a, b] \times[0, \infty)\right)$ модуль непрерывности отображения $\Delta:[a, b] \times C^{n}[a, b] \rightarrow \operatorname{comp}\left[\mathbb{R}^{n}\right]$, порождающего оператор $\Phi: C^{n}[a, b] \rightarrow \Pi\left[L^{n}[a, b]\right]$, если при этом при почти всех $t \in[a, b]$ отображение $\Delta(t, \cdot)$ непрерывно на $U$.

Отметим также, что, подбирая радиус непрерывности $\omega(\cdot, \cdot)$, модуль непрерывности отображения $\Delta(\cdot, \cdot)$ можно сделать “достаточно малым" в каждой точке $(x, \delta) \in U \times(0, \infty)$. В связи с этим функция $\lambda_{U}(\omega)(\cdot, \cdot)$, определенная равенством (96), будет “сколь угодно малой” в каждой точке $\delta>0$.

ЗАмЕчАНИЕ 17. Как было отмечено ранее для оператора Немьщкого

$$
\mathscr{N}: C^{n}[a, b] \rightarrow \Pi\left[L^{n}[a, b]\right]
$$


порождающее его отображение $\Delta_{1}:[a, b] \times C^{n}[a, b] \rightarrow \operatorname{comp}\left[\mathbb{R}^{n}\right]$ можно рассматривать на произведении $[a, b] \times \mathbb{R}^{n}$ (это сужение здесь обозначается как отображение $F:[a, b] \times \mathbb{R}^{n} \rightarrow \operatorname{comp}\left[\mathbb{R}^{n}\right]$ ). Так как $\mathbb{R}^{n} \subset C^{n}[a, b]$ (каждый элемент пространства $\mathbb{R}^{n}$ рассматривается как постоянная функция) и каждый замкнутый шар пространства $\mathbb{R}^{n}$ является компактом в пространстве $C^{n}[a, b]$, то функцию $\varphi_{U}(\omega):[a, b] \times U \times[0, \infty) \rightarrow[0, \infty)$, определенную равенством $(91)$, можно задать на всем пространстве $\mathbb{R}^{n}$ с помощью соотношения

$$
\varphi(\omega)(t, x, \delta)=\max _{y \in B[x ; \omega(x, \delta)]} h[F(t, x) ; F(t, y)]
$$

При этом функция $\varphi(\omega):[a, b] \times \mathbb{R}^{n} \times[0, \infty) \rightarrow[0, \infty)$, определенная равенством (99), обладает свойствами а)-d) (см. лемму 12) на любом ограниченном множестве $U \subset \mathbb{R}^{n}$. Поэтому в этом случае можно уточнить следствие 5 , которое гарантирует существование хотя бы одной функции $\eta(\cdot, \cdot) \in K([a, b] \times[0, \infty))$, равномерно на множестве $U \subset \mathbb{R}^{n}$ оценивающей сверху относительно фиксированного радиуса непрерывности $\omega(\cdot, \cdot) \in P\left(\mathbb{R}^{n} \times[0, \infty)\right)$ модуль непрерывности отображения $F:[a, b] \times \mathbb{R}^{n} \rightarrow \operatorname{comp}\left[\mathbb{R}^{n}\right]$, порождаюшего оператор Немыцкого $\mathscr{N}: C^{n}[a, b] \rightarrow \Pi\left[L^{n}[a, b]\right]$. Следствие 5 в этом случае остается справедливым без предположения выпуклости множества $U \subset \mathbb{R}^{n}$.

Пусть $\eta(\cdot, \cdot) \in K([a, b] \times[0, \infty))$ и $\xi(\cdot, \cdot) \in P\left(C^{n}[a, b] \times[0, \infty)\right)$. Рассмотрим для каждого $\delta>0$ в пространстве $C^{n}[a, b]$ включение

$$
x \in(\Psi(x))^{\xi(x, \delta)}+V \Phi_{\eta}(x, \delta),
$$

где отображение $\Phi_{\eta}: C^{n}[a, b] \times[0, \infty) \rightarrow \Pi\left[L^{n}[a, b]\right]$ задано соотношениями $(82)$, (85). Включение (100) будем изучать во взаимосвязи с включениями (7) и (38).

Каждое решение включения (100) при фиксированном $\delta>0$ будем называть $\delta$-решением включения (приближенным решением) (7). Через $H_{\eta(\delta), \xi(\delta)}(U)$ обозначим множество всех $\delta$-решений включения (7), принадлежащих множеству $U \subset C^{n}[a, b]$. Обозначим множества решений включений $(7),(38)$, принадлежаших множеству $U \subset C^{n}[a, b]$, через $H(U), H_{\text {со }}(U)$ соответственно.

TЕОРема 7. Пусть $U$ - непустое замкнутое множество пространства $C^{n}[a, b]$ и $\xi(\cdot, \cdot) \in P\left(C^{n}[a, b] \times[0, \infty)\right)$. Тогда для любой функиии $\eta(\cdot, \cdot) \in$ $K([a, b] \times[0, \infty))$, равномерно на множсестве $U \subset C^{n}[a, b]$ оценивающей сверху относительно радиуса непрерьвности $\omega(\cdot, \cdot) \in P\left(C^{n}[a, b] \times[0, \infty)\right)$ модуль непрерывности отображсения $\Delta:[a, b] \times C^{n}[a, b] \rightarrow \operatorname{comp}\left[\mathbb{R}^{n}\right]$, порожсдающего оператор $\Phi: C^{n}[a, b] \rightarrow \Pi\left[L^{n}[a, b]\right]$, справедливо равенство

$$
H_{\mathrm{co}}(U)=\bigcap_{\delta>0} \overline{H_{\eta(\delta), \xi(\delta)}(U)}
$$

әде $\overline{H_{\eta(\delta), \xi(\delta)}(U)}-$ замыкание в $C^{n}[a, b]$ множества $H_{\eta(\delta), \xi(\delta)}(U)$. 
ДокАЗАТЕльство. Вначале отметим, что поскольку множество $U$ замкнуто в $C^{n}[a, b]$, то согласно теореме 4 множество $H_{\text {со }}(U)$ замкнуто в $C^{n}[a, b]$.

Далее, докажем вложение

$$
H_{\mathrm{co}}(U) \subset \bigcap_{\delta>0} \overline{H_{\eta(\delta), \xi(\delta)}(U)}
$$

Пусть $x \in H_{\text {со }}(U)$. Покажем, что для любого $\delta>0 x$-предельная точка множества $H_{\eta(\delta), \xi(\delta)}(U)$. Действительно, согласно теореме $2 x$ - квазирешение включения (7), причем $x \in U$. Поэтому в силу сделанного ранее предположения о квазирешении найдутся такой элемент $v \in \Psi(x)$ и такая последовательность $z_{i} \in \Phi(x)$, $i=1,2, \ldots$, что последовательность $x_{i}=v+V z_{i} \rightarrow x$ в $C^{n}[a, b]$ при $i \rightarrow \infty$ и $x_{i} \in U$ для любого $i=1,2, \ldots$. Далее, пусть $\eta(\cdot, \cdot) \in K([a, b] \times[0, \infty))$ равномерно на множестве $U$ оценивает сверху модуль непрерывности отображения $\Delta(\cdot, \cdot)$ относительно радиуса непрерьвности $\omega(\cdot, \cdot) \in P\left(C^{n}[a, b] \times[0, \infty)\right)$. Тогда найдется $i_{1}$ такое, что при всех $i \geqslant i_{1}$ выполняется неравенство $\left\|x-x_{i}\right\|_{C^{n}[a, b]}<\omega(x, \delta)$. Это означает, что при всех $i \geqslant i_{1}$ справедливо включение $x_{i} \in \overline{B_{C^{n}[a, b]}[x ; \omega(x, \delta)]}$. Поэтому для любого $i \geqslant i_{1} x_{i} \in M_{U}(\omega)(x, \delta)$. Далее, из определения равномерной оценки сверху модуля непрерьвности отображения $\Delta(\cdot, \cdot)$ следует существование такого числа $i_{2} \geqslant i_{1}$, что для любого $i \geqslant i_{2}$ при почти всех $t \in[a, b]$ имеет место неравенство

$$
\varphi_{U}(\omega)\left(t, x,\left\|x-x_{i}\right\|_{C^{n}[a, b]}\right) \leqslant \eta(t, \delta) .
$$

Из неравенства (103) при всех $i \geqslant i_{2}$ и при почти всех $t \in[a, b]$ получаем оценки

$$
\rho\left[z_{i}(t) ; \Delta\left(t, x_{i}\right)\right] \leqslant h\left[\Delta(t, x) ; \Delta\left(t, x_{i}\right)\right] \leqslant \varphi_{U}(\omega)\left(t, x,\left\|x-x_{i}\right\|_{C^{n}[a, b]}\right) \leqslant \eta(t, \delta) .
$$

Далее, так как $\xi\left(x_{i}, \delta\right) \rightarrow \xi(x, \delta)>0$ при $i \rightarrow \infty$, то найдется $i_{3} \geqslant i_{2}$ такое, что при всех $i \geqslant i_{3}$ выполняется оценка

$$
h_{C^{n}[a, b]}\left[\Psi(x) ; \Psi\left(x_{i}\right)\right] \leqslant \xi\left(x_{i}, \delta\right) .
$$

Из неравенства (105) следует, что при всех $i \geqslant i_{3}$ имеет место соотношение

$$
\rho_{C^{n}[a, b]}\left[v ; \Psi\left(x_{i}\right)\right] \leqslant \xi\left(x_{i}, \delta\right) .
$$

Из (104) и (106) вытекает, что для любого $i \geqslant i_{3}$ справедливо включение $x_{i} \in$ $H_{\eta(\delta), \xi(\delta)}(U)$. Это означает, что $x$ - предельная точка множества $H_{\eta(\delta), \xi(\delta)}(U)$. Поэтому $x \in \overline{H_{\eta(\delta), \xi(\delta)}(U)}$ и, следовательно, вложение (102) доказано.

Докажем теперь обратное включение, т.е.

$$
\bigcap_{\delta>0} \overline{H_{\eta(\delta), \xi(\delta)}(U)} \subset H_{\mathrm{co}}(U) .
$$

Пусть

$$
x \in \bigcap_{\delta>0} \overline{H_{\eta(\delta), \xi(\delta)}(U)} .
$$


Это означает, что для любого $i=1,2, \ldots$ найдется $x_{i} \in H_{\eta(1 / i), \xi(1 / i)}(U)$, удовлетворяюший неравенству $\left\|x-x_{i}\right\|_{C^{n}[a, b]}<1 / i$. Пусть для каждого $i=1,2, \ldots$ функции $v_{i} \in \Psi\left(x_{i}\right)^{\xi\left(x_{i}, 1 / i\right)}, z_{i} \in \Phi_{\eta}\left(x_{i}, 1 / i\right)$ такие, что

$$
x_{i}=v_{i}+V z_{i}
$$

Далее, пусть для каждого $i=1,2, \ldots$ функция $\widetilde{v}_{i} \in \Psi(x)$ удовлетворяет равенству

$$
\left\|v_{i}-\widetilde{v}_{i}\right\|_{C^{n}[a, b]}=\rho_{C^{n}[a, b]}\left[v_{i} ; \Psi(x)\right],
$$

а для функции $\widetilde{z}_{i} \in \Phi(x)$ при почти всех $t \in[a, b]$ справедливо соотношение

$$
\left|z_{i}(t)-\widetilde{z}_{i}(t)\right|=\rho\left[z_{i}(t) ; \Delta(t, x)\right] .
$$

Так как $\Psi(x)$ компактно, то, не уменьшая обшности, можно считать, что $\widetilde{v}_{i} \rightarrow v \in$ $\Psi(x)$ в пространстве $C^{n}[a, b]$ при $i \rightarrow \infty$. Докажем, что $v_{i} \rightarrow v$ в $C^{n}[a, b]$ при $i \rightarrow \infty$. Действительно, из равенства (108) для любого $i=1,2, \ldots$ вытекают оценки

$\left\|v_{i}-\widetilde{v}_{i}\right\|_{C^{n}[a, b]} \leqslant h_{C^{n}[a, b]}\left[\Psi\left(x_{i}\right)^{\xi\left(x_{i}, 1 / i\right)} ; \Psi(x)\right] \leqslant \xi\left(x_{i}, \frac{1}{i}\right)+h_{C^{n}[a, b]}\left[\Psi\left(x_{i}\right) ; \Psi(x)\right]$, из которых следует, что $\lim _{i \rightarrow \infty}\left\|v_{i}-\widetilde{v}_{i}\right\|_{C^{n}[a, b]}=0$. А это означает, что $v_{i} \rightarrow v$ в $C^{n}[a, b]$ при $i \rightarrow \infty$.

Теперь докажем, что

$$
\lim _{i \rightarrow \infty}\left\|z_{i}-\widetilde{z}_{i}\right\|_{L^{n}[a, b]}=0 .
$$

Действительно, так как $\eta(\cdot, \cdot) \in K([a, b] \times[0, \infty))$, то в силу теоремы Лебега

$$
\lim _{i \rightarrow \infty} \int_{a}^{b} \eta\left(t, \frac{1}{i}\right) d t=0 .
$$

Далее, из равенства (109) для любого $i=1,2, \ldots$ при почти всех $t \in[a, b]$ получаем соотношения

$$
\left|z_{i}(t)-\widetilde{z}_{i}(t)\right| \leqslant h\left[\left(\Delta\left(t, x_{i}\right)\right)^{\eta(t, 1 / i)} ; \Delta(t, x)\right] \leqslant \eta\left(t, \frac{1}{i}\right)+h\left[\Delta(t, x) ; \Delta\left(t, x_{i}\right)\right] .
$$

Поэтому из (109) для любого $i=1,2, \ldots$ вытекает

$$
\int_{a}^{b}\left|z_{i}(t)-\widetilde{z}_{i}(t)\right| d t \leqslant \int_{a}^{b} \eta\left(t, \frac{1}{i}\right) d t+2 h_{L^{n}[a, b]}\left[\Phi(x) ; \Phi\left(x_{i}\right)\right] .
$$

Следовательно, согласно (111) и непрерьвности отображения $\Phi: C^{n}[a, b] \rightarrow$ $\Pi\left[L^{n}[a, b]\right]$ получаем соотношение (110).

Далее, так как последовательность $\widetilde{z}_{i}, i=1,2, \ldots$, - слабо предкомпактное множество пространства $L^{n}[a, b]$, то, не уменьшая общности, будем считать, что $\widetilde{z}_{i} \rightarrow z$ слабо в $L^{n}[a, b]$ при $i \rightarrow \infty$. Согласно $[8] z \in \overline{\mathrm{co}}(\Phi(x))$. Из равенства (110) следует, что $z_{i} \rightarrow z$ слабо в пространстве $L^{n}[a, b]$ при $i \rightarrow \infty$. Следовательно, не уменьшая обшности, можно считать, что $V z_{i} \rightarrow V z$ в $C^{n}[a, b]$ при $i \rightarrow \infty$. Поэтому, переходя в равенствах (107) к пределу при $i \rightarrow \infty$, получаем соотношение $x=v+V z$, где $v \in \Psi(x), z \in \overline{\mathrm{co}}(\Phi(x))$, т.е. $x \in H_{\text {со }}(U)$. Таким образом, представление (101) справедливо. Теорема доказана. 
ЗАмЕчАнИЕ 18. Отметим, что дифференциальные включения являются частньм случаем возмущенных включений. А так как для дифференциального включения равенство $\overline{H(U)}=H_{\text {со }}(U)$ может не вьполняться (см., например, [14], [20]), то из теоремы 7 (см. следствие 5 и замечание 16) следует, что соотношение

$$
\overline{\bigcap_{\delta>0} H_{\eta(\delta), \xi(\delta)}(U)}=\bigcap_{\delta>0} \overline{H_{\eta(\delta), \xi(\delta)}(U)}
$$

может при некоторых $(\eta(\cdot, \cdot), \xi(\cdot, \cdot)) \in K([a, b] \times[0, \infty)) \times P\left(C^{n}[a, b] \times[0, \infty)\right)$ не иметь места.

Кроме того, из теоремы 7 и замечания 16 вытекает следующий, на наш взгляд, важньй факт. Если предположить, что отображение $\Phi: C^{n}[a, b] \rightarrow \Pi\left[L^{n}[a, b]\right]$ подвергается "небольшим возмушениям" (значения $\Phi(\cdot)$ определяются со сколь угодно мальми погрешностями), то эти неточности определения значений отображения $\Phi(\cdot)$ приводят включение (7) к “овыпукленному включению” (38).

ОПРЕДЕЛЕНИЕ. Пусть $U \subset C^{n}[a, b]$. По аналогии с [16], [31] будем говорить, что для включения (7) на множестве $U \subset C^{n}[a, b]$ выполняется принцип nлотности (условие плотности), если справедливо равенство

$$
\overline{H(U)}=H_{\mathrm{co}}(U) \text {. }
$$

ЗАмЕчАнИЕ 19. Как было отмечено выше, принцип плотности не всегда выполняется. Это доказывает пример Плиса (см. [14], [20]). Первые достаточные условия, когда выполняется равенство (112), для задачи Коши дифференциального включения получены А.Ф. Филипповым (см. [10]-[12]), а для периодических решений и для краевых задач эти условия опубликованы в работах [5], [6], [10], [17], [30].

Tеорема 8. Пусть $\xi(\cdot, \cdot) \in P\left(C^{n}[a, b] \times[0, \infty)\right)$. Если $U-$ непустое замкнутое множество пространства $C^{n}[a, b]$, то для выполнения равенства

$$
\overline{H(U)}=\bigcap_{\delta>0} \overline{H_{\eta(\delta), \xi(\delta)}(U)}
$$

для любого радиуса внешних возмущений $\eta(\cdot, \cdot) \in K([a, b] \times[0, \infty))$ достаточно, а если $U$ - непустое выпуклое компактное множество пространства $C^{n}[a, b]$, то и необходимо выполнение принципа плотности на множестве $U \subset C^{n}[a, b]$.

ДокаЗАТЕЛЬство.Докажем вначале достаточность. Пусть на множестве $U \subset$ $C^{n}[a, b]$ выполняется принцип плотности. Покажем, что для $(\eta(\cdot, \cdot), \xi(\cdot, \cdot)) \in$ $K([a, b] \times[0, \infty)) \times P\left(C^{n}[a, b] \times[0, \infty)\right)$ выполняется равенство (113). Действительно, из определения включения (100) вытекает, что для любого $\delta>0$ справедливо соотношение

$$
H(U) \subset H_{\eta(\delta), \xi(\delta)}(U) .
$$

Поэтому для любого $\delta>0$ выполняется включение

$$
\overline{H(U)} \subset \overline{H_{\eta(\delta), \xi(\delta)}(U)}
$$


и, следовательно, имеет место вложение

$$
\overline{H(U)} \subset \bigcap_{\delta>0} \overline{H_{\eta(\delta), \xi(\delta)}(U)} .
$$

Теперь покажем, что справедливо обратное включение

$$
\bigcap_{\delta>0} \overline{H_{\eta(\delta), \xi(\delta)}(U)} \subset \overline{H(U)},
$$

которое в силу равенства (112) эквивалентно вложению

$$
\bigcap_{\delta>0} \overline{H_{\eta(\delta), \xi(\delta)}(U)} \subset H_{\mathrm{co}}(U) .
$$

Последнее соотношение доказывается аналогично тому, как это было сделано при доказательстве теоремы 7.

Если $U$ - непустое выпуклое компактное множество, то необходимость вьполнения равенства $(113)$ для любых $\eta(\cdot, \cdot) \in K([a, b] \times[0, \infty))$ непосредственно вытекает из следствия 5 и замечания 16 . Теорема доказана.

ЗАмечАниЕ 20. Отметим, что выполнение равенства (113) для любых внешних возмущений $(\eta(\cdot, \cdot), \xi(\cdot, \cdot)) \in K([a, b] \times[0, \infty)) \times P\left(C^{n}[a, b] \times[0, \infty)\right)$ является свойством устойчивости множества решений $H(U)$ включения $(7)$ относительно этих возмущений (см. [16], [31]).

СлеДСтвие 6. Пусть отображения $V: L^{n}[a, b] \rightarrow C^{n}[a, b], \Psi: C^{n}[a, b] \rightarrow$ $\operatorname{comp}\left[C^{n}[a, b]\right], \Phi: C^{n}[a, b] \rightarrow \Pi\left[L^{n}[a, b]\right]$ обладают свойством В. Тогда для любых $(\eta(\cdot, \cdot), \xi(\cdot, \cdot)) \in K([a, b] \times[0, \infty)) \times P\left(C^{n}[a, b] \times[0, \infty)\right)$ выполняется равенство (113) на множестве $U=C^{n}[a, b]$.

Действительно, так как согласно определениям вьполняются равенства $H=$ $H\left(C^{n}[a, b]\right)$ и $H_{\text {со }}=H_{\text {со }}\left(C^{n}[a, b]\right)$, то согласно теореме 5 для включения (7) на пространстве $C^{n}[a, b]$ выполняется принцип плотности. Поэтому в силу теоремы 8 равенство (113) выполняется для любых $(\eta(\cdot, \cdot), \xi(\cdot, \cdot)) \in K([a, b] \times[0, \infty)) \times$ $P\left(C^{n}[a, b] \times[0, \infty)\right)$.

2.2. Возмущенное включение с внешними и внутренними возмущениями. Как уже отмечалось в п. 2.1, внешние возмушения характеризуют погрешность вычислений значений многозначных отображений

$$
\Psi: C^{n}[a, b] \rightarrow \operatorname{comp}\left[C^{n}[a, b]\right] \quad \text { и } \quad \Phi: C^{n}[a, b] \rightarrow \Pi\left[L^{n}[a, b]\right] .
$$

В то же время каждое решение $x:[a, b] \rightarrow \mathbb{R}^{n}$ включения (1) может вычисляться в этих многозначных отображениях с некоторыми степенями точности, которые можно задать некоторыми функциями из множества $P\left(C^{n}[a, b] \times[0, \infty)\right)$. Эту неточность определения решения можно учесть с помощью внутренних возмушений.

Для того чтобы определить аппроксимирующее отображение оператора $\Phi$ : $C^{n}[a, b] \rightarrow \Pi\left[L^{n}[a, b]\right]$, учитываюшее не только погрешность вычислений значений, но и погрешность вычисления решения (внутренние возмушения), нам потребуется следуюшее утверждение. 
Лемма 13. Пусть $U$ - компактное множсество пространства $C^{n}[a, b]$, $\eta(\cdot, \cdot) \in K([a, b] \times[0, \infty))$. Тогда для любого $\tau \in[0, \infty)$ мнохество $\Phi_{\eta}(U,[0, \tau])$ - слабо предкомпактное множсество пространства $L^{n}[a, b]$, где отображение $\Phi_{\eta}: C^{n}[a, b] \times[0, \infty) \rightarrow \Pi\left[L^{n}[a, b]\right]$ определено соотношениями $(82),(85)$.

ДоКАЗАТЕЛЬСТво. Прежде всего, аналогично доказательству леммы 3 можно показать, что если $U$ - компактное множество, то образ $\Phi(U)$ - слабо предкомпактное множество пространства $L^{n}[a, b]$. Поэтому для любого $\varepsilon>0$ сушествует $\delta>0$ такое, что для любого измеримого множества $e \subset[a, b]$, удовлетворяющего неравенству $\mu е<\delta$, вьполняются неравенства

$$
\int_{e}|x(s)| d s<\varepsilon, \quad \int_{e} \beta_{\tau}(s) d s<\varepsilon
$$

где первое неравенство в (114) выполняется для любой функции $x \in \Phi(U)$, а второе - для суммируемой функции $\beta_{\tau}(\cdot)$, которая определяет множество $K([a, b] \times$ $[0, \infty))$. Из определения отображения $\Phi_{\eta}: C^{n}[a, b] \times[0, \infty) \rightarrow \Pi\left[L^{n}[a, b]\right]$ (cм. (82), $(85))$ для каждой функции $y \in \Phi_{\eta}(U,[0, \tau])$ найдется такая функция $x \in \Phi(U)$, для которой при почти всех $t \in[a, b]$ справедливы оценки

$$
|y(t)| \leqslant|x(t)|+|y(t)-x(t)| \leqslant|x(t)|+\beta_{\tau}(t)
$$

Из соотношений (115) и (114) вытекает слабая предкомпактность множества $\Phi_{\eta}(U,[0, \tau])$. Лемма доказана.

Пусть $U$ - вьпуклое компактное множество пространства $C^{n}[a, b]$, и пусть $\eta(\cdot, \cdot) \in K([a, b] \times[0, \infty))$ и $\sigma(\cdot, \cdot), \theta(\cdot, \cdot) \in P\left(C^{n}[a, b] \times[0, \infty)\right)$. Определим отображения $\Psi_{\sigma}: U \times[0, \infty) \rightarrow \operatorname{comp}\left[C^{n}[a, b]\right], \Phi_{\eta, \theta}: U \times[0, \infty) \rightarrow \operatorname{comp}\left[L^{n}[a, b]^{*}\right]$ равенствами

$$
\begin{aligned}
\Psi_{\sigma}(x, \delta) & =\Psi\left(M_{U}(\sigma)(x, \delta)\right), \\
\Phi_{\eta, \theta}(x, \delta) & =\Phi_{\eta}\left(M_{U}(\theta)(x, \delta), \delta\right),
\end{aligned}
$$

где отображения $M_{U}(\sigma): U \times[0, \infty) \rightarrow \Omega(U), M_{U}(\theta): U \times[0, \infty) \rightarrow \Omega(U)$ заданы равенством $(90)$, в котором $\omega(\cdot, \cdot)$ соответственно равны $\sigma(\cdot, \cdot)$ и $\theta(\cdot, \cdot)$, отображение $\Phi_{\eta}: C^{n}[a, b] \times[0, \infty) \rightarrow \Pi\left[L^{n}[a, b]\right]$ определено соотношениями (82), (85). Далее, пусть отображения ext $\Delta_{\eta}:[a, b] \times C^{n}[a, b] \times[0, \infty) \rightarrow \operatorname{comp}\left[\mathbb{R}^{n}\right], \operatorname{ext} \Phi_{\eta}$ : $C^{n}[a, b] \times[0, \infty) \rightarrow \Pi\left[L^{n}[a, b]\right], \operatorname{ext} \Phi_{\eta, \theta}: U \times[0, \infty) \rightarrow \operatorname{comp}\left[L^{n}[a, b]^{*}\right]$ имеют вид

$$
\begin{aligned}
\left(\operatorname{ext} \Delta_{\eta}\right)(t, x, \delta) & =((\operatorname{ext} \Delta)(t, x))^{\eta^{(t, \delta)}} \\
\left(\operatorname{ext} \Phi_{\eta}\right)(x, \delta) & =S\left(\left(\operatorname{ext} \Delta_{\eta}\right)(\cdot, x, \delta)\right) \\
\left(\operatorname{ext} \Phi_{\eta, \theta}\right)(x, \delta) & =\left(\operatorname{ext} \Phi_{\eta}\right)\left(M_{U}(\theta)(x, \delta), \delta\right),
\end{aligned}
$$

где отображение ext $\Delta:[a, b] \times C^{n}[a, b] \rightarrow \operatorname{comp}\left[\mathbb{R}^{n}\right]$ задано соотношением (48). 
ЗАмЕчАНИЕ 21. Отметим, что объединение выпуклых по переключению множеств может быть невыпуклым по переключению множеством, поэтому значения отображений $\Phi_{\eta, \theta}(\cdot, \cdot), \operatorname{ext} \Phi_{\eta, \theta}(\cdot, \cdot \cdot)$ могут не принадлежать множеству $\Pi\left[L^{n}[a, b]\right]$.

На множестве $U \subset C^{n}[a, b]$ для каждого $\delta>0$ рассмотрим включения

$$
\begin{aligned}
& x \in\left(\Psi_{\sigma}(x, \delta)\right)^{\xi(x, \delta)}+V \Phi_{\eta, \theta}(x, \delta), \\
& x \in\left(\Psi_{\sigma}(x, \delta)\right)^{\xi(x, \delta)}+V\left(\operatorname{ext} \Phi_{\eta, \theta}\right)(x, \delta),
\end{aligned}
$$

где $\xi(\cdot, \cdot) \in P\left(C^{n}[a, b] \times[0, \infty)\right)$, отображения $\Psi_{\sigma}: U \times[0, \infty) \rightarrow \operatorname{comp}\left[C^{n}[a, b]\right]$, $\Phi_{\eta, \theta}: U \times[0, \infty) \rightarrow \operatorname{comp}\left[L^{n}[a, b]^{*}\right], \operatorname{ext} \Phi_{\eta, \theta}: U \times[0, \infty) \rightarrow \operatorname{comp}\left[L^{n}[a, b]^{*}\right]$ заданы равенствами (116), (117), (90), (118)-(120), (48).

При каждом $\delta>0$ через $H_{\eta(\delta), \theta(\delta), \sigma(\delta), \xi(\delta)}(U)$, ext $H_{\eta(\delta), \theta(\delta), \sigma(\delta), \xi(\delta)}(U)$ обозначим множества решений включений (121), (122), принадлежащих множеству $U \subset C^{n}[a, b]$. Так как для любого $\delta>0$ и любого $x \in U$ выполняется включение $\left(\operatorname{ext} \Phi_{\eta, \theta}\right)(x, \delta) \subset \Phi_{\eta, \theta}(x, \delta)$, то для любого $\delta>0$ справедливо вложение ext $H_{\eta(\delta), \theta(\delta), \sigma(\delta), \xi(\delta)}(U) \subset H_{\eta(\delta), \theta(\delta), \sigma(\delta), \xi(\delta)}(U)$.

Далее, предположим, что квазирешения включения (51), принадлежащие множеству $U \subset C^{n}[a, b]$, обладают тем же свойством, что и квазирешения включения (7), т.е. если $x$ - квазирешение включения (51) и $x \in U \subset C^{n}[a, b]$, то найдутся такой элемент $v \in \Psi(x)$ и такая последовательность $z_{i} \in(\operatorname{ext} \Phi)(x), i=1,2, \ldots$, что для любого $i=1,2, \ldots$ выполняется включение $x_{i}=v+V z_{i} \in U$ и $x_{i} \rightarrow x$ в $C^{n}[a, b]$ при $i \rightarrow \infty$.

Как ранее отмечено, для многих задач (например, периодических, многоточечных краевых задач) это свойство квазирешений включения (51) можно доказать непосредственно (см. [6]), используя теорему 2 и следствие 3 . Кроме того, если оператор, определенный правой частью включения (7), переводит выпуклое замкнутое множество $U \subset C^{n}[a, b]$ в себя, то согласно теореме 2 и следствию 3 квазирешения включения (51) обладают вышеупомянутым свойством на множестве $U$.

ТЕОРема 9. Пусть $U$ - выпуклое компактное множество пространства $C^{n}[a, b]$. Тогда для любых $\eta(\cdot, \cdot) \in K([a, b] \times[0, \infty)), \theta(\cdot, \cdot), \sigma(\cdot, \cdot), \xi(\cdot, \cdot) \in$ $P\left(C^{n}[a, b] \times[0, \infty)\right)$ справедливы равенства

$$
H_{\mathrm{co}}(U)=\bigcap_{\delta>0} \overline{\operatorname{ext} H_{\eta(\delta), \theta(\delta), \sigma(\delta), \xi(\delta)}(U)}=\bigcap_{\delta>0} \overline{H_{\eta(\delta), \theta(\delta), \sigma(\delta), \xi(\delta)}(U)},
$$

где $\overline{\operatorname{ext} H_{\eta(\delta), \theta(\delta), \sigma(\delta), \xi(\delta)}(U)}, \overline{H_{\eta(\delta), \theta(\delta), \sigma(\delta), \xi(\delta)}(U)}$ - замыкания в пространстве $C^{n}[a, b]$ множсеств решений $\operatorname{ext} H_{\eta(\delta), \theta(\delta), \sigma(\delta), \xi(\delta)}(U), H_{\eta(\delta), \theta(\delta), \sigma(\delta), \xi(\delta)}(U)$ соответственно.

ДокАЗАТЕльство. Вначале покажем, что

$$
H_{\mathrm{co}}(U) \subset \bigcap_{\delta>0} \overline{\operatorname{ext} H_{\eta(\delta), \theta(\delta), \sigma(\delta), \xi(\delta)}(U)} .
$$


Пусть $x \in H_{\text {со }}(U)$. Покажем, что для любого $\delta>0 x$-предельная точка множества ext $H_{\eta(\delta), \theta(\delta), \sigma(\delta), \xi(\delta)}(U)$. Действительно, согласно сделанному вьше предположению и следствию 2 найдутся такой элемент $v \in \Psi(x)$ и такая последовательность $z_{i} \in(\operatorname{ext} \Phi)(x), i=1,2, \ldots$, что для любого $i=1,2, \ldots$ вьполняется включение $x_{i}=v+V z_{i} \in U$ и $x_{i} \rightarrow x$ в $C^{n}[a, b]$ при $i \rightarrow \infty$. Далее, докажем, что найдется такой номер $I$, что при всех $i \geqslant I$

$$
x_{i} \in \operatorname{ext} H_{\eta(\delta), \theta(\delta), \sigma(\delta), \xi(\delta)}(U) .
$$

Так как $\theta(\cdot, \cdot), \sigma(\cdot, \cdot) \in P\left(C^{n}[a, b] \times[0, \infty)\right)$, то найдется такой номер $i_{0}$, что при всех $i \geqslant i_{0}$ выполняются включения

$$
x \in B_{C^{n}[a, b]}\left[x_{i} ; \theta\left(x_{i}, \delta\right)\right], \quad x \in B_{C^{n}[a, b]}\left[x_{i} ; \sigma\left(x_{i}, \delta\right)\right] .
$$

А это означает, что для любого $i \geqslant i_{0} x \in M_{U}(\theta)\left(x_{i}, \delta\right)$ и $x \in M_{U}(\sigma)\left(x_{i}, \delta\right)$. Поэтому для любого $i \geqslant i_{0}$ выполняются вложения

$$
\Psi(x) \subset \Psi_{\sigma}\left(x_{i}, \delta\right), \quad(\operatorname{ext} \Phi)(x) \subset\left(\operatorname{ext} \Phi_{\eta, \theta}\right)\left(x_{i}, \delta\right)
$$

Из соотношений (126) и определения включения (122) вытекает, что при всех $i \geqslant$ $I=i_{0}$ выполняется включение (125), а это означает, что точка $x$ - предельная точка множества ext $H_{\eta(\delta), \theta(\delta), \sigma(\delta), \xi(\delta)}(U)$, т.е. $x \in \overline{\operatorname{ext} H_{\eta(\delta), \theta(\delta), \sigma(\delta), \xi(\delta)}(U)}$ и, следовательно, вложение (124) справедливо.

Вложение

$$
\bigcap_{\delta>0} \overline{\operatorname{ext} H_{\eta(\delta), \theta(\delta), \sigma(\delta), \xi(\delta)}(U)} \subset H_{\mathrm{co}}(U)
$$

доказывается аналогично соответствующему включению в доказательстве теоремы 7. Второе равенство (123) доказывается аналогично. Теорема доказана.

ЗАмечАниЕ 22. Теорема 9 доказывает, что при наличии внутренних возмущений в правой части включения (7) никакая точность вычислений значений отображения $\Delta:[a, b] \times C^{n}[a, b] \rightarrow \operatorname{comp}\left[\mathbb{R}^{n}\right]$, порождающего оператор $\Phi: C^{n}[a, b] \rightarrow$ $\Pi\left[L^{n}[a, b]\right]$, не гарантирует "восстановление" множества $\overline{H(U)}$ по замыканиям в пространстве $C^{n}[a, b]$ множеств приближенных решений. Это возможно только в одном случае, когда выполняется принцип плотности.

\section{§ 3. Краевые задачи функционально-дифференциальных включений}

3.1. Возмущение линейной краевой задачи для функционально-дифференциальных уравнений. Здесь для общего вида линейной краевой задачи функционально-дифференциальной системы уравнений определяется общая возмущенная краевая задача, которая состоит из функционально-дифференциального включения, определяемого возмущениями линейной функционально-дифференциальной системы уравнений, и из включения для краевых условий, связанных возмущениями линейного вектор-функционала.

Рассмотрим линейный непрерывный оператор $\mathscr{L}: D^{n}[a, b] \rightarrow L^{n}[a, b]$. Запишем отображение $\mathscr{L}$ в виде

$$
\mathscr{L} x=Q \dot{x}+A(\cdot) x(a),
$$


где оператор $Q: L^{n}[a, b] \rightarrow L^{n}[a, b]$ (главная часть оператора $\mathscr{L}$ в представлении (127), см. [39]) определяется равенством $Q=\mathscr{L} \Lambda$, оператор $\Lambda: L^{n}[a, b] \rightarrow$ $D^{n}[a, b]$ - оператор интегрирования, каждый столбец $n \times n$ матрицы $A(t)$ представляет собой результат применения оператора $\mathscr{L}$ к соответствующему столбцу единичной матрицы: $A(t)=(\mathscr{L} E)(t)$. Будем предполагать, что оператор $Q$ имеет обратный и обратный оператор $Q^{-1}: L^{n}[a, b] \rightarrow L^{n}[a, b]$ непрерывен.

Отметим, что этот класс линейных отображений содержит линейные дифференциальные операторы вида

$$
(\mathscr{L} x)(t)=\dot{x}(t)+\mathscr{P}(t) x(t)
$$

где отображение $\mathscr{P}:[a, b] \rightarrow \mathbb{R}^{n \times n}$ имеет суммируемые на $[a, b]$ элементы; дифференциальные отображения с операторами внутренней суперпозиции, интегро-дифференциальные операторы и др.

Рассмотрим линейную краевую задачу для функционально-дифференциального уравнения

$$
\mathscr{L} x=0, \quad l x=0,
$$

где $l: D^{n}[a, b] \rightarrow \mathbb{R}^{n}$ - линейный непрерьвньй вектор-функционал.

Будем предполагать, что краевая задача (128) имеет только нулевое решение. В этом случае согласно [39] существует непрерывный оператор Грина $G: L^{n}[a, b] \rightarrow$ $D^{n}[a, b]$, определенный равенством

$$
(G z)(t)=\int_{a}^{b} G(t, s) z(s) d s, \quad t \in[a, b],
$$

который для произвольного $z \in L^{n}[a, b]$ решение $x \in D^{n}[a, b]$ краевой задачи

$$
\mathscr{L} x=z, \quad l x=0,
$$

представляет в виде $x=G z$ и, наоборот, каждое значение $G z$-решение задачи (130).

Пусть $X:[a, b] \rightarrow \mathbb{R}^{n \times n}$ - фундаментальная матрица решений первого уравнения (128), удовлетворяющая условию

$$
l(X)=E
$$

$(E$ - единичная матрица, матрица $l(X)$ представляет собой результат применения вектор-функционала $l$ к соответствующему столбцу матрицы $X$ ). В этом случае краевую задачу

$$
\mathscr{L} x=z, \quad l x=c,
$$

где $z \in L^{n}[a, b], c \in \mathbb{R}^{n}$, можно представить в виде

$$
x=X c+G z .
$$


Отметим, что равенства (132) в реальных математических моделях выполняются с какой-то степенњю точности. Кроме того, сами линейные операторы

$$
\mathscr{L}: D^{n}[a, b] \rightarrow L^{n}[a, b], \quad l: D^{n}[a, b] \rightarrow \mathbb{R}^{n}
$$

определяются для различных процессов с теми или иными допущениями и предположениями, которые используют либо неполноту информации о реальном исследуемом процессе, либо “простоту" описания самой математической модели этого процесса. В связи с этими обстоятельствами целесообразно рассмотреть включения

$$
\mathscr{L} x \in \Phi(x), \quad l x \in \varphi(x),
$$

в которых многозначные отображения $\Phi: C^{n}[a, b] \rightarrow \Pi\left[L^{n}[a, b]\right], \varphi: C^{n}[a, b] \rightarrow$ $\operatorname{comp}\left[\mathbb{R}^{n}\right]$ могут описать неточность информации о процессе, различного рода предположения и допущения, а также степень аппроксимации математической модели этого процесса.

Задачу (134) будем называть квазилинейной краевой задачей для функиионально-дифференциальных включений или просто возмущенной краевой задачей.

Будем говорить, что функция $x \in D^{n}[a, b]$ - решение задачи (134), если $x$ удовлетворяет и первому, и второму включениям в (134).

Рассмотрим интегральное включение

$$
x \in X \varphi(x)+G \Phi(x)
$$

где $X$ - фундаментальная матрица решений первого уравнения (128), удовлетворяющая равенству (131), отображение $G: L^{n}[a, b] \rightarrow D^{n}[a, b]$ - оператор Грина, определенный соотношением (129).

ЛЕмма 14. Возмущенная краевая задача (134) әквивалентна интегральному включению (135). Любое решение $x$ включения (135) однозначно представимо в виде (133), где $c \in \varphi(x), z \in \Phi(x)$.

ДоказАтЕльство. Действительно, пусть $x$ - решение включения (135). Тогда найдутся $c \in \varphi(x)$ и $z \in \Phi(x)$, для которых справедливо равенство (133). Поэтому согласно [39] получаем, что $x-X c$ удовлетворяет равенствам $\mathscr{L}(x-X c)=z$, $l(x-X c)=0$. Из определения матрицы $X$ вытекает, что $\mathscr{L} x=z$ и $l x=c$, т.е. $x-$ решение задачи (134). Обратное утверждение доказывается аналогично.

Докажем теперь, что любое решение $x$ включения (135) однозначно представимо в виде (133). Предположим противное, т.е. для решения $x$ включения (135) найдутся такие $c_{1}, c_{2} \in \varphi(x)$ и $z_{1}, z_{2} \in \Phi(x)$, для которых справедливы равенства

$$
x=X c_{1}+G z_{1}=X c_{2}+G z_{2}
$$

Отсюда получаем, что $X\left(c_{1}-c_{2}\right)=G\left(z_{1}-z_{2}\right)$ - решение задачи (130) для $z=$ $z_{2}-z_{1}$. Поэтому $l\left(X\left(c_{1}-c_{2}\right)\right)=0$ и, следовательно, $l\left(X\left(c_{1}-c_{2}\right)\right)=E\left(c_{1}-c_{2}\right)=0$, т.е. $c_{1}=c_{2}$. Таким образом, $G\left(z_{1}-z_{2}\right)=0$ и поэтому $z_{1}=z_{2}$. Лемма доказана. 
ЗАмЕчАниЕ 23. Таким образом, лемма 14 утверждает, что множество решений включения (135) разложимо с точки зрения определения, сформулированного в 11 .

Докажем следуюшую лемму, которая потребуется нам в дальнейшем для исследования возмущенных краевых задач.

Лемма 15. Оператор Грина $G: L^{n}[a, b] \rightarrow D^{n}[a, b]$, определенный равенством (129), переводит каждое слабо предкомпактное множество пространства $L^{n}[a, b]$ в предкомпактное множество пространства $C^{n}[a, b]$.

ДокАЗАТЕльство. Так как оператор дифференцирования $d / d t: D^{n}[a, b] \rightarrow$ $L^{n}[a, b]$ является линейным непрерывным отображением, то он переводит каждую слабо сходяшуюся последовательность в слабо сходяшуюся. Поэтому в силу представления

$$
G f=\Lambda \frac{d}{d t}(G f)+(G f)(a)
$$

где $\Lambda$ - оператор интегрирования, а $f \in L^{n}[a, b]$, достаточно доказать, что если последовательность $f_{i}\left(\in L^{n}[a, b]\right), i=1,2, \ldots$, слабо сходится к функции $f_{0}\left(\in L^{n}[a, b]\right)$ в пространстве $L^{n}[a, b]$ при $i \rightarrow \infty$, то $\Lambda f_{i} \rightarrow \Lambda f_{0}$ в пространстве $C^{n}[a, b]$ при $i \rightarrow \infty$. Докажем это. Пусть $\varepsilon>0$. Поскольку последовательность $f_{i}, i=1,2, \ldots$, слабо сходится в $L^{n}[a, b]$, то она имеет равностепенно абсолютно непрерьвные интегралы. Это означает, что найдется такое $\delta(\varepsilon)>0$, что для любого измеримого множества $e \subset[a, b]$, удовлетворяющего неравенству $\mu e<\delta(\varepsilon)$, для любого $i=0,1,2, \ldots$ вьполняется оценка

$$
\int_{e}\left|f_{i}(s)\right| d s<\varepsilon
$$

Разобьем отрезок $[a, b]$ на $m$ равных по мере частей точками

$$
a=t_{0}^{m}<t_{1}^{m}<\cdots<t_{m-1}^{m}<t_{m}^{m}=b,
$$

удовлетворяющими неравенствам $t_{j+1}^{m}-t_{j}^{m}<\delta(\varepsilon), j=0,1, \ldots, m-1$. Так как последовательность $\Lambda f_{i} \rightarrow \Lambda f_{0}$ слабо в пространстве $C^{n}[a, b]$ при $i \rightarrow \infty$, то найдется такой номер $I(\varepsilon)$, что при всех $i \geqslant I(\varepsilon)$ и всех $j=1, \ldots, m$ вьполняется оценка

$$
\left|\left(\Lambda f_{i}\right)\left(t_{j}^{m}\right)-\left(\Lambda f_{0}\right)\left(t_{j}^{m}\right)\right|<\varepsilon .
$$

Далее, пусть $t \in[a, b]$. Это означает, что $t \in\left[t_{j}^{m}, t_{j+1}^{m}\right]$ при некотором $j=$ $0,1, \ldots, m-1$. Поэтому из оценок (136), (137) для любого $i \geqslant I(\varepsilon)$ получаем соотношения

$$
\begin{gathered}
\left|\left(\Lambda f_{i}\right)(t)-\left(\Lambda f_{0}\right)(t)\right| \leqslant\left|\int_{t_{i}^{m}}^{t}\left(f_{i}(s)-f_{0}(s)\right) d s\right|+\left|\int_{a}^{t_{i}^{m}}\left(f_{i}(s)-f_{0}(s)\right) d s\right| \\
\leqslant \int_{t_{i}^{m}}^{t}\left|f_{i}(s)\right| d s+\int_{t_{i}^{m}}^{t}\left|f_{0}(s)\right| d s+\left|\int_{a}^{t_{i}^{m}}\left(f_{i}(s)-f_{0}(s)\right) d s\right|<3 \varepsilon .
\end{gathered}
$$

Это означает, что $\Lambda f_{i} \rightarrow \Lambda f_{0}$ в пространстве $C^{n}[a, b]$ при $i \rightarrow \infty$. Лемма доказана. 
3.2. Квазилинейные краевые задачи для функционально-дифференциальных включений. Здесь изучим задачу (134) на основе полученных в $§ 1$ результатов.

Пусть $q_{0} \in C^{n}[a, b], r_{0} \in \varphi\left(q_{0}\right)$ и $w_{0} \in L^{n}[a, b]$. Представим функцию $q_{0}$ равенством

$$
q_{0}=X r_{0}+G w_{0}+e
$$

где $e=X r_{0}-G w_{0}$. Пусть непрерывная функция $\nu:[a, b] \rightarrow[0, \infty)$ определена соотношением

$$
\nu(t)=\int_{a}^{b}|G(t, s)| k(s) d s+|e(t)|
$$

где $|G(t, s)|$ - согласованная с пространством $\mathbb{R}^{n}$ норма $n \times n$ матрицы $G(t, s)$ в представлении (129), $e \in C^{n}[a, b]$ - функция из правой части равенства (138), функция $k \in L^{1}[a, b]$ для любого измеримого множества $\mathscr{U} \subset[a, b]$ удовлетворяет неравенству (10).

ОПРЕДЕЛЕНИЕ. Будем Говорить, что оператор Грина $G: L^{n}[a, b] \rightarrow C^{n}[a, b]$ и многозначные отображения $\varphi: C^{n}[a, b] \rightarrow \operatorname{comp}\left[\mathbb{R}^{n}\right], \Phi: C^{n}[a, b] \rightarrow \Pi\left[L^{n}[a, b]\right]$ обладают свойством $\widetilde{\mathrm{A}}$, если найдутся непрерывные изотонные операторы $\Gamma$ : $C_{+}^{1}[a, b] \rightarrow L_{+}^{1}[a, b]$ и $P: C_{+}^{1}[a, b] \rightarrow \mathbb{R}^{1}$, удовлетворяющие следующим условиям: для любых $x, y \in C^{n}[a, b]$ и любого измеримого множества $\mathscr{U} \subset[a, b]$ выполняется соотношение $(12)$ и для любых $x, y \in C^{n}[a, b]$ справедлива оценка

$$
h[\varphi(x) ; \varphi(y)] \leqslant P(Z(x-y))
$$

для функции $\nu \in C_{+}^{1}[a, b]$, определенной соотношением (139), сходится в пространстве $C^{1}[a, b]$ ряд

$$
\sum_{i=0}^{\infty} \widetilde{\mathscr{A}}^{i} \nu, \quad \widetilde{\mathscr{A}}^{0} \nu=\nu, \quad \widetilde{\mathscr{A}}^{i} \nu=\widetilde{\mathscr{A}}\left(\widetilde{\mathscr{A}}^{i-1} \nu\right), \quad i=1,2, \ldots
$$

где непрерывный оператор $\tilde{\mathscr{A}}: C_{+}^{1}[a, b] \rightarrow C_{+}^{1}[a, b]$ определен равенством

$$
\begin{aligned}
(\widetilde{\mathscr{A}} z)(t) & =\int_{a}^{b}|G(t, s)|(\Gamma z)(s) d s+\lambda P(z), \\
\lambda & =\max \{|X(t)|: t \in[a, b]\}
\end{aligned}
$$

отображение $Z: C^{n}[a, b] \rightarrow C_{+}^{1}[a, b]$ имеет вид (16).

Пусть $\widetilde{\xi}(\nu)$ - сумма ряда (141), т.е.

$$
\widetilde{\xi}(\nu)=\sum_{i=0}^{\infty} \widetilde{\mathscr{A}}^{i} \nu
$$


ОПРеДЕЛЕНИЕ. Будем говорить, что оператор Грина $G: L^{n}[a, b] \rightarrow C^{n}[a, b]$ и многозначные отображения $\varphi: C^{n}[a, b] \rightarrow \operatorname{comp}\left[\mathbb{R}^{n}\right], \Phi: C^{n}[a, b] \rightarrow \Pi\left[L^{n}[a, b]\right]$ обладают свойством $\widetilde{\mathrm{A}}^{*}$, если найдутся непрерьвные изотонные операторы $\Gamma: C_{+}^{1}[a, b] \rightarrow L_{+}^{1}[a, b]$ и $P: C_{+}^{1}[a, b] \rightarrow \mathbb{R}^{1}$, удовлетворяющие неравенствам (12), $(140)$, а также соотношениям $\Gamma(0)=0, P(0)=0$, и, кроме того, для любой функции $\widetilde{\nu} \in C_{+}^{1}[a, b]$ из некоторой окрестности нуля ряд (141) сходится в пространстве $C^{1}[a, b]$ и сумма этого ряда непрерывна в нуле.

Teоpema 10. Пусть $q_{0} \in C^{n}[a, b], r_{0} \in \varphi\left(q_{0}\right)$ u $w_{0} \in L^{n}[a, b]$, и пусть функция qо представима в виде (138). Далее, пусть оператор Грина $G: L^{n}[a, b] \rightarrow$ $C^{n}[a, b]$ и многозначные отображения $\varphi: C^{n}[a, b] \rightarrow \operatorname{comp}\left[\mathbb{R}^{n}\right], \Phi: C^{n}[a, b] \rightarrow$ $\Pi\left[L^{n}[a, b]\right]$ обладают свойством А․ Тогда найдется такое решение $x$ задачи (134), для которого выполняются следующие оценки: при любом $t \in[a, b]$

$$
\begin{aligned}
\left|x(t)-q_{0}(t)\right| & \leqslant \widetilde{\xi}(\nu)(t), \\
\left\|X\left(r_{0}-l x\right)\right\|_{C^{n}[a, b]} & \leqslant \lambda P(\widetilde{\xi}(\nu)),
\end{aligned}
$$

при почти всех $t \in[a, b]$

$$
\left|(\mathscr{L} x)(t)-w_{0}(t)\right| \leqslant k(t)+\Gamma(\widetilde{\xi}(\nu))(t),
$$

где функции $\nu, \widetilde{\xi}(\nu)$, иисло $\lambda$ определень соотношениями (139), (144), (143), функция $k$ удовлетворяет неравенству (10), для отображений Г и Р выполняются оценки (12), (140).

Действительно, задача (134) эквивалентна включению (135), при этом отображения $G: L^{n}[a, b] \rightarrow C^{n}[a, b], \widetilde{\Psi}: C^{n}[a, b] \rightarrow \operatorname{comp}\left[C^{n}[a, b]\right], \Phi: C^{n}[a, b] \underset{\widetilde{\Psi}}{\longrightarrow}$ $\Pi\left[L^{n}[a, b]\right]$ обладают свойством А, сформулированным в $\S 1$, где отображение $\widetilde{\Psi}$ имеет вид $\widetilde{\Psi} y=X \varphi(y)$, которое для любых $x, y \in C^{n}[a, b]$ удовлетворяет неравенству $h_{C^{n}[a, b]}[\widetilde{\Psi}(x) ; \widetilde{\Psi}(y)] \leqslant \lambda P(Z(x-y))$, здесь число $\lambda$ определено равенством (143). Поэтому теорема 10 является следствием теоремы 1.

ОПРЕДЕЛЕНИЕ. Будем говорить, что оператор $G: L^{n}[a, b] \rightarrow C^{n}[a, b]$ и многозначные отображения $\varphi: C^{n}[a, b] \rightarrow \operatorname{comp}\left[\mathbb{R}^{n}\right], \Phi: C^{n}[a, b] \rightarrow \Pi\left[L^{n}[a, b]\right]$ обладают свойством $\widetilde{\mathrm{B}}$, если выполняются следующие условия: найдется функция $\beta \in L^{1}[a, b]$ такая, что для любых $x, y \in C^{n}[a, b]$ и любого измеримого множества $\mathscr{U} \subset[a, b]$ вьполняется неравенство

$$
h_{L^{n}(\mathscr{U})}[\Phi(x) ; \Phi(y)] \leqslant \int_{\mathscr{U}} \beta(s) d s\|x-y\|_{C^{n}[a, b]} ;
$$

найдется число $\alpha \geqslant 0$ такое, что для любых $x, y \in C^{n}[a, b]$ функционал $\varphi$ удовлетворяет неравенству

$$
h[\varphi(x) ; \varphi(y)] \leqslant \alpha\|x-y\|_{C^{n}[a, b]}
$$

для функции $\beta \in L^{1}[a, b]$ и числа $\alpha \geqslant 0$ справедливо соотношение

$$
\max _{t \in[a, b]} \int_{a}^{b}|G(t, s)| \beta(s) d s+\alpha \lambda<1
$$


где число $\lambda$ определено равенством (143).

Далее, пусть непрерывный оператор $\widetilde{\mathscr{B}}: C_{+}^{1}[a, b] \rightarrow C_{+}^{1}[a, b]$ имеет вид

$$
(\mathscr{B} z)(t)=\left(\int_{a}^{b}|G(t, s)| \beta(s) d s+\alpha \lambda\right)\|z\|_{C^{1}[a, b]} .
$$

Пусть непрерывная функция $\nu:[a, b] \rightarrow[0, \infty)$ определена соотношением (139). Обозначим

$$
\widetilde{\xi_{0}}(\nu)=\sum_{i=0}^{\infty} \mathscr{B}^{i} \nu, \quad \mathscr{B}^{0} \nu=\nu, \quad \mathscr{B}^{i} \nu=\mathscr{B}\left(\mathscr{B}^{i-1} \nu\right), \quad i=1,2, \ldots
$$

Так как для любого $i=0,1, \ldots$ выполняется оценка

$$
\left\|\mathscr{B}^{i} \nu\right\|_{C^{1}[a, b]} \leqslant \zeta^{i}\|\nu\|_{C^{1}[a, b]},
$$

где

$$
\zeta=\max _{t \in[a, b]} \int_{a}^{b}|G(t, s)| \beta(s) d s+\alpha \lambda,
$$

и согласно неравенству $(149) \zeta<1$, то ряд $(151)$ сходится в пространстве $C^{1}[a, b]$.

Таким образом, из теоремы 10 вытекает

СЛЕДСТВИЕ 7. Пусть $q_{0} \in C^{n}[a, b], r_{0} \in \varphi\left(q_{0}\right)$ u $w_{0} \in L^{n}[a, b]$, u nyсmь функиия $q_{0}$ представима равенством (138). Далее, пусть оператор Грина $G: L^{n}[a, b] \rightarrow C^{n}[a, b]$ и многозначные отображсения $\varphi: C^{n}[a, b] \rightarrow \operatorname{comp}\left[\mathbb{R}^{n}\right]$, $\Phi: C^{n}[a, b] \rightarrow \Pi\left[L^{n}[a, b]\right]$ обладают свойством $\widetilde{\mathrm{B}}$. Тогда найдется такое решение $x$ задачи (134), для которого выполняются следующие оценки:

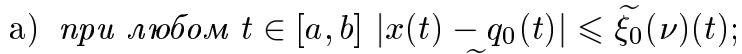

b) $\left\|X\left(r_{0}-l x\right)\right\|_{C^{n}[a, b]} \leqslant \lambda \alpha\left\|\widetilde{\xi_{0}}(\nu)\right\|_{C^{1}[a, b]}$;

c) при почти всех $t \in[a, b]$ справедливо соотношение

$$
|(\mathscr{L} x)(t)-w(t)| \leqslant k(t)+\beta(t)\left\|\widetilde{\xi_{0}}(\nu)\right\|_{C^{1}[a, b]}
$$

где функции $\nu, \widetilde{\xi_{0}}(\nu)$, число $\lambda$ определень соотноиениями (139), (150), (143), число $\alpha$, функичи $k, \beta$ удовлетворяют неравенствам (148), (10), (147).

ЗАмечаниЕ 24 . Если $\mathscr{L} x=\dot{x}, l x=x(a), \varphi(x)=x_{0}\left(x_{0} \in \mathbb{R}^{n}\right), \Phi$ - оператор Немыцкого, то оценки, установленные в теореме 10 и следствии 7 , аналогичны оценкам, опубликованным в работах [11], [12], [14], [18], [23], [25], [40], [41]. Кроме того, эти оценки дополняют оценки в работах $[3],[5]$, поскольку не предполагают вьпуклозначности отображения $\varphi$.

Рассмотрим вместе с задачей (134) и задачи

$$
\begin{array}{ll}
\mathscr{L} x \in \overline{\operatorname{co}}(\Phi(x)), & l x \in \varphi(x), \\
\mathscr{L} x \in(\operatorname{ext} \Phi)(x), & l x \in \varphi(x),
\end{array}
$$


где многозначный оператор ext $\Phi: C^{n}[a, b] \rightarrow \Pi\left[L^{n}[a, b]\right]$ определен равенствами (48), (49). Пусть $H^{1}, H_{\mathrm{co}}^{1}, H_{\mathrm{ext}}^{1}$ - множества всех решений задач $(134),(154)$, (155) соответственно.

Так как согласно лемме 14 задачи (154), (155) эквивалентны включениям

$$
x \in X \varphi(x)+G(\overline{\operatorname{co}}(\Phi(x))), \quad x \in X \varphi(x)+G((\operatorname{ext} \Phi)(x)),
$$

где $G$ - оператор Грина, матрица $X$ определена в п. 3.1, то из лемм 14,15 и теоремы 6 вытекает

Tеорема 11. Пусть оператор $G: L^{n}[a, b] \rightarrow C^{n}[a, b]$ и многозначные отображения $\varphi: C^{n}[a, b] \rightarrow \operatorname{comp}\left[\mathbb{R}^{n}\right], \Phi: C^{n}[a, b] \rightarrow \Pi\left[L^{n}[a, b]\right]$ обладают свойством $\widetilde{\mathrm{A}}^{*}$. Тогда $H_{\mathrm{ext}}^{1} \neq \varnothing$ и выполняются равенства

$$
\overline{H_{\mathrm{ext}}^{1}}=\overline{H^{1}}=H_{\mathrm{co}}^{1}
$$

əде $\overline{H_{\mathrm{ext}}^{1}}, \overline{H^{1}}$ - замыкания множеств $H_{\mathrm{ext}}^{1}, H^{1}$ в пространстве $C^{n}[a, b]$ соответственно.

СлеДСТВИЕ 8. Пусть оператор Грина $G: L^{n}[a, b] \rightarrow C^{n}[a, b]$ и многозначньие отображения $\varphi: C^{n}[a, b] \rightarrow \operatorname{comp}\left[\mathbb{R}^{n}\right], \Phi: C^{n}[a, b] \rightarrow \Pi\left[L^{n}[a, b]\right]$ обладают свойством $\widetilde{\mathrm{B}}$. Тогда $H_{\mathrm{ext}}^{1} \neq \varnothing$ и выполняется равенство (156).

Действительно, согласно неравенству (149) для любой функции $\widetilde{\nu} \in C_{+}^{1}[a, b]$ сумму $\widetilde{\xi_{0}}(\widetilde{\nu})$ ряда (151) можно оценить следуюшим образом:

$$
\left\|\widetilde{\xi_{0}}(\widetilde{\nu})\right\|_{C^{1}[a, b]} \leqslant \frac{1}{1-\zeta}\|\widetilde{\nu}\|_{C^{1}[a, b]}
$$

где число $\zeta$ определено равенством (153). Поэтому сумма $\widetilde{\xi_{0}}(\widetilde{\nu})$ непрерывна в точке 0 и следствие справедливо.

ЗАмечАниЕ 25. Теорема 11 и следствие 7 устанавливают достаточные условия, при которых для задачи (134) выполняется “бэнг-бэнг” принцип.

ЗАмЕчАниЕ 26. Отметим, что известные результаты о плотности множеств решений дифференциальных включений с невыпуклой и овыпукленной правыми частями и о “бэнг-бэнг” принципе (см. обзоры и монографии [11], [14], [18], [40]-[46], а также работы [12], [21]-[28], [47]-[49]) касаются только задачи Коши для дифференциальных включений и предполагают в той или иной форме вольтерровость отображений $\mathscr{L}$ и $\Phi$. В теореме 11 и следствии 8 вольтерровость этих отображений не предполагается. Кроме того, эти утверждения распространяют результаты работ [6], [11], [18] на случай, когда линейный вектор-функционал $l$ возмушен многозначным вектор-функционалом $\varphi$, а также распространяют результаты работ [3], [5] на случай, когда многозначный вектор-функционал $\varphi$ имеет невыпуклые образы. 
3.3. Дифференциальные включения с многозначным оператором Немыщкого. Здесь рассматриваются дифференциальные включения с многозначньм оператором Немыцкого. В этом случае можно получить мажорантные неравенства, параметры которых иногда представляют собой решения линейных интегральных уравнений.

Рассмотрим краевую задачу

$$
(\mathscr{L} x)(t) \in F(t, x(t)), \quad t \in[a, b], \quad l x \in \varphi(x)
$$

где линейные непрерывные операторы $\mathscr{L}: D^{n}[a, b] \rightarrow L^{n}[a, b], l: D^{n}[a, b] \rightarrow \mathbb{R}^{n}$ и многозначное отображение $\varphi: C^{n}[a, b] \rightarrow \operatorname{comp}\left[\mathbb{R}^{n}\right]$ удовлетворяют упомянутым вьшше условиям. Многозначная функция $F:[a, b] \times \mathbb{R}^{n} \rightarrow \operatorname{comp}\left[\mathbb{R}^{n}\right]$ удовлетворяет следуюшим условиям: найдется неотрицательная функция $\beta \in L^{1}[a, b]$ такая, что для любых $x, y \in \mathbb{R}^{n}$ при почти всех $t \in[a, b]$ выполняется неравенство

$$
h[F(t, x) ; F(t, y)] \leqslant \beta(t)|x-y|
$$

для любого $x \in \mathbb{R}^{n}$ отображение $F(\cdot, x)$ измеримо; функция $p:[a, b] \rightarrow[0, \infty)$, определенная равенством $p(t)=\|F(t, 0)\|$, суммируемая.

ОПРЕДЕЛЕНИЕ. Будем говорить, что оператор Грина $G: L^{n}[a, b] \rightarrow C^{n}[a, b]$ и многозначные отображения $\varphi: C^{n}[a, b] \rightarrow \operatorname{comp}\left[\mathbb{R}^{n}\right], \mathscr{N}: C^{n}[a, b] \rightarrow \Pi\left[L^{n}[a, b]\right]$ oбладают свойством $\mathrm{C}$, если найдется число $\alpha \geqslant 0$ такое, что для любых $x, y \in$ $C^{n}[a, b]$ функционал $\varphi$ удовлетворяет неравенству (148); для непрерьвного оператора $\widetilde{\mathscr{B}}: C_{+}^{1}[a, b] \rightarrow C_{+}^{1}[a, b]$, определенного равенством

$$
(\widetilde{\mathscr{B}} z)(t)=\int_{a}^{b}|G(t, s)| \beta(s) z(s) d s+\alpha \lambda\|z\|_{C^{1}[a, b]},
$$

сходится в пространстве $C^{1}[a, b]$ ряд

$$
\sum_{i=0}^{\infty} \widetilde{\mathscr{B}}^{i} \nu, \quad \widetilde{\mathscr{B}}^{0} \nu=\nu, \quad \widetilde{\mathscr{B}}^{i} \nu=\widetilde{\mathscr{B}}\left(\widetilde{\mathscr{B}}^{i-1} \nu\right), \quad i=1,2, \ldots
$$

где функция $\nu \in C^{1}[a, b]$ определена соотношением (139), число $\lambda$ задано равенством (143), функция $\beta \in L^{1}[a, b]$ удовлетворяет неравенству (158).

Пусть $\widetilde{\xi_{1}}(\nu)$ - сумма ряда $(160)$, т.е.

$$
\widetilde{\xi_{1}}(\nu)=\sum_{i=0}^{\infty} \mathscr{B}^{i} \nu
$$

Из теоремы 10 вытекает 
Teopema 12. Пyсmb $q_{0} \in C^{n}[a, b], r_{0} \in \varphi\left(q_{0}\right) u w_{0} \in L^{n}[a, b]$, u nycmb функция $q_{0}$ представима равенством (138). Далее, пусть оператор Грина $G: L^{n}[a, b] \rightarrow C^{n}[a, b]$ и многозначные отображения $\varphi: C^{n}[a, b] \rightarrow \operatorname{comp}\left[\mathbb{R}^{n}\right]$, $\mathscr{N}: C^{n}[a, b] \rightarrow \Pi\left[L^{n}[a, b]\right]$ обладают свойством С. Тогда найдется такое решение $x$ задачи (134), для которого выполняются следующие оценки:

a) при любом $t \in[a, b]\left|x(t)-q_{0}(t)\right| \leqslant \widetilde{\xi_{1}}(\nu)(t)$;

b) $\left\|X\left(r_{0}-l x\right)\right\|_{C^{n}[a, b]} \leqslant \lambda \alpha\left\|\widetilde{\xi}_{1}(\nu)\right\|_{C^{1}[a, b]}$;

c) при почти всех $t \in[a, b]|(\mathscr{L} x)(t)-w(t)| \leqslant k(t)+\beta(t) \widetilde{\xi_{1}}(\nu)(t)$,

где матрица $X$ определена в $n .3 .1$, функции $\nu, \widetilde{\xi}_{1}(\nu)$, число $\lambda$ заданьи соотношениями (139), (161), (143), функиия $k$ удовлетворяет неравенству (10), в котором $\Phi$ - оператор Немыцкого $\mathscr{N}$, порожденный отображением $F:[a, b] \times$ $\mathbb{R}^{n} \rightarrow \operatorname{comp}\left[\mathbb{R}^{n}\right]$.

ЗАмечание 27. Отметим, что если $\mathscr{L} x=\dot{x}$ и задача (134) - задача Коши $\left(l x=x(a), \varphi(x)=x_{0}, x_{0} \in \mathbb{R}^{n}\right)$, то в этом случае матрица $G(t, s)$, которая порождает оператор Грина задачи Коши, определяется равенствами

$$
G(t, s)= \begin{cases}E, & a \leqslant s \leqslant t \leqslant b \\ 0, & a \leqslant t<s \leqslant b\end{cases}
$$

где $E$ - единичная матрица, а 0 - нулевая матрица. И, следовательно, в этом случае оператор $\widetilde{\mathscr{B}}$, заданный равенством $(159)$, в котором $\alpha=0$, представляет собой оператор Вольтерра, поэтому ряд (160) сходится для любой функции $\beta \in L^{1}[a, b]$, удовлетворяюшей неравенству (158). Поэтому теорема 12 содержит в себе результат А. Ф. Филиппова (см. [11], [12], [14]).

Рассмотрим краевые задачи

$$
\begin{array}{lll}
(\mathscr{L} x)(t) \in \operatorname{co}(F(t, x(t))), & t \in[a, b], & l x \in \varphi(x), \\
(\mathscr{L} x)(t) \in \overline{\operatorname{ext}}(\operatorname{co}(F(t, x(t)))), & t \in[a, b], & l x \in \varphi(x) .
\end{array}
$$

Пусть $H^{2}, H_{\text {co }}^{2}, H_{\text {ext }}^{2}$ - множества всех решений задач $(157),(162),(163)$ соответственно.

ОПРЕДЕЛЕНИЕ. Будем говорить, что оператор Грина $G: L^{n}[a, b] \rightarrow C^{n}[a, b]$ и многозначные отображения $\varphi: C^{n}[a, b] \rightarrow \operatorname{comp}\left[\mathbb{R}^{n}\right], \mathscr{N}: C^{n}[a, b] \rightarrow \Pi\left[L^{n}[a, b]\right]$ oбладают свойством $\mathrm{C}^{*}$, если они обладают свойством $\mathrm{C}$ и для любой функции $\widetilde{\nu} \in C_{+}^{1}$ из некоторой окрестности нуля ряд (160) сходится и сумма ряда непрерывна в точке нуль.

Tеорема 13. Пусть оператор Грина $G: L^{n}[a, b] \rightarrow C^{n}[a, b]$ и многозначные отображения $\varphi: C^{n}[a, b] \rightarrow \operatorname{comp}\left[\mathbb{R}^{n}\right], \mathscr{N}: C^{n}[a, b] \rightarrow \Pi\left[L^{n}[a, b]\right]$ обладают свойством $\mathrm{C}^{*}$. Тогда $H_{\mathrm{ext}}^{2} \neq \varnothing$ и виполняется равенство $\overline{H_{\mathrm{ext}}^{2}}=\overline{H^{2}}=H_{\mathrm{co}}^{2}$, где $\overline{H_{\mathrm{ext}}^{2}}, \overline{H^{2}}$ - замыкания множеств $H_{\mathrm{ext}}^{2}, H^{2}$ в пространстве $C^{n}[a, b]$. 
3.4. Квазилинейные краевые задачи функционально-дифференциальных включений с внутренними и внешними возмущениями. Будем предполагать, что отображение $\Delta:[a, b] \times C^{n}[a, b] \rightarrow \operatorname{comp}\left[\mathbb{R}^{n}\right]$, порождающее отображение $\Phi: C^{n}[a, b] \rightarrow \Pi\left[L^{n}[a, b]\right]$, при почти всех $t \in[a, b]$ непрерьвно по второму аргументу на пространстве $C^{n}[a, b]$, а отображение $\varphi: C^{n}[a, b] \rightarrow \operatorname{comp}\left[\mathbb{R}^{n}\right]$ непрерывно по Хаусдорфу. Пусть $\xi(\cdot, \cdot) \in P\left(C^{n}[a, b] \times[0, \infty)\right), \eta(\cdot, \cdot) \in K([a, b] \times$ $[0, \infty))$. Рассмотрим для каждого $\delta>0$ задачу

$$
\mathscr{L} x \in \Phi_{\eta}(x, \delta), \quad l x \in \varphi(x)^{\xi(x, \delta)},
$$

где многозначное отображение $\Phi_{\eta}: C^{n}[a, b] \times[0, \infty) \rightarrow \Pi\left[L^{n}[a, b]\right]$ определено равенствами (82), (85).

Задачу (164) будем называть возмущенной краевой задачей с внешними возмущениями.

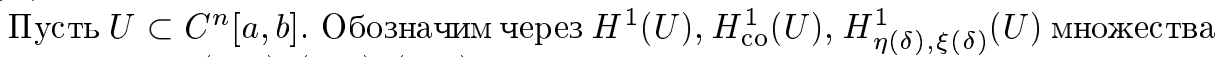
решений задач $(134),(154),(164)$, принадлежащих множеству $U$, соответственно.

ОПРЕДЕЛЕНИЕ. Будем говорить, что для задачи (134) на множестве $U \subset$ $C^{n}[a, b]$ выполняется принцип плотности (условие плотности), если справедливо равенство

$$
\overline{H^{1}(U)}=H_{\mathrm{co}}^{1}(U)
$$

где $\overline{H^{1}(U)}$ - замькание в пространстве $C^{n}[a, b]$ множества $H^{1}(U)$.

Так как для любого $\delta>0$ задача (164) эквивалентна включению

$$
x \in X \varphi(x)^{\xi(x, \delta)}+G \Phi_{\eta}(x, \delta),
$$

где $G: L^{n}[a, b] \rightarrow C^{n}[a, b]$ - оператор Грина, матрица $X$ определена в п. 3.1 , то из теорем 7,8 вытекают следующие утверждения.

Teоpema 14. Пусть $U$ - непустое замкнутое множество пространства $C^{n}[a, b]$ и $\xi(\cdot, \cdot) \in P\left(C^{n}[a, b] \times[0, \infty)\right)$. Тогда для любой функиии $\eta(\cdot, \cdot) \in$ $K([a, b] \times[0, \infty))$, равномерно на множестве $U \subset C^{n}[a, b]$ оченивающей сверху относительно радиуса непрерывности $\omega(\cdot, \cdot) \in P\left(C^{n}[a, b] \times[0, \infty)\right)$ модуль непрерывности отображения $\Delta:[a, b] \times C^{n}[a, b] \rightarrow \operatorname{comp}\left[\mathbb{R}^{n}\right]$, порождающего оператор $\Phi: C^{n}[a, b] \rightarrow \Pi\left[L^{n}[a, b]\right]$, справедливо равенство

$$
H_{\mathrm{co}}^{1}(U)=\bigcap_{\delta>0} \overline{H_{\eta(\delta), \xi(\delta)}^{1}(U)},
$$

где $\overline{H_{\eta(\delta), \xi(\delta)}^{1}(U)}$ - замыкание в $C^{n}[a, b]$ множества $H_{\eta(\delta), \xi(\delta)}^{1}(U)$.

Teорема 15. Пусть $\xi(\cdot, \cdot) \in P\left(C^{n}[a, b] \times[0, \infty)\right)$. Если U - непустое замкнутое множество пространства $C^{n}[a, b]$, то для выполнения равенства

$$
\overline{H^{1}(U)}=\bigcap_{\delta>0} \overline{H_{\eta(\delta), \xi(\delta)}^{1}(U)}
$$

для любого радиуса внешних возмущений $\eta(\cdot, \cdot) \in K([a, b] \times[0, \infty))$ достаточно, а если $U$ - непустое выпуклое компактное множество пространства $C^{n}[a, b]$, то и необходимо выполнение принципа плотности на множестве $U \subset C^{n}[a, b]$. 
СлеДСТВИЕ 9. Пусть оператор Грина $G: L^{n}[a, b] \rightarrow C^{n}[a, b]$ и многозначнье отображсения $\varphi: C^{n}[a, b] \rightarrow \operatorname{comp}\left[\mathbb{R}^{n}\right], \Phi: C^{n}[a, b] \rightarrow \Pi\left[L^{n}[a, b]\right]$ обладают свойством $\widetilde{\mathrm{A}}^{*}$. Тогда для любых $(\eta(\cdot, \cdot), \xi(\cdot, \cdot)) \in K([a, b] \times[0, \infty)) \times$ $P\left(C^{n}[a, b] \times[0, \infty)\right)$ выполняется равенство (165) на множестве $U=C^{n}[a, b]$.

Пусть $U$ - вьпуклое компактное множество пространства $C^{n}[a, b]$, и пусть $\eta(\cdot, \cdot) \in K([a, b] \times[0, \infty))$ и $\sigma(\cdot, \cdot), \theta(\cdot, \cdot) \in P\left(C^{n}[a, b] \times[0, \infty)\right)$. На множестве $U \subset C^{n}[a, b]$ для каждого $\delta>0$ рассмотрим задачи

$$
\begin{array}{ll}
\mathscr{L} x \in \Phi_{\eta, \theta}(x, \delta), & l x \in \varphi_{\sigma}(x, \delta)^{\xi(x, \delta)}, \\
\mathscr{L} x \in\left(\operatorname{ext} \Phi_{\eta, \theta}\right)(x, \delta), & l x \in \varphi_{\sigma}(x, \delta)^{\xi(x, \delta)},
\end{array}
$$

где $\xi(\cdot, \cdot) \in P\left(C^{n}[a, b] \times[0, \infty)\right)$, отображения $\Phi_{\eta, \theta}: U \times[0, \infty) \rightarrow \operatorname{comp}\left[L^{n}[a, b]^{*}\right]$, $\operatorname{ext} \Phi_{\eta, \theta}: U \times[0, \infty) \rightarrow \operatorname{comp}\left[L^{n}[a, b]^{*}\right]$ определены равенствами (117), (82), (85), $(90),(118)-(120),(48)$, отображение $\varphi_{\sigma}: U \times[0, \infty) \rightarrow \operatorname{comp}\left[\mathbb{R}^{n}\right]$ имеет вид

$$
\varphi_{\sigma}(x, \delta)=\varphi\left(M_{U}(\sigma)(x, \delta)\right)
$$

здесь отображение $M_{U}(\sigma): U \times[0, \infty) \rightarrow \Omega(U)$ задано равенством $(90)$, в котором $\omega(\cdot, \cdot)$ соответствует $\sigma(\cdot, \cdot)$.

Задачу (166) будем называть возмущенной краевой задачей с внутренними и внешними возмущениями, а задачу (167) будем называть возмущенной краевой задачей с внутренними и внешними возмущениями, построенной по крайним точкам отображения $\Phi: C^{n}[a, b] \rightarrow \Pi\left[L^{n}[a, b]\right]$.

При каждом $\delta>0$ через $H_{\eta(\delta), \theta(\delta), \sigma(\delta), \xi(\delta)}^{1}(U)$, ext $H_{\eta(\delta), \theta(\delta), \sigma(\delta), \xi(\delta)}^{1}(U)$ обозначим множества решений задач (166), (167), принадлежащих множеству $U \subset$ $C^{n}[a, b]$.

Так как для любого $\delta>0$ задачи (166) и (167) эквивалентны включениям

$$
\begin{aligned}
& x \in X\left(\varphi_{\sigma}(x, \delta)\right)^{\xi(x, \delta)}+G \Phi_{\eta, \theta}(x, \delta), \\
& x \in X\left(\varphi_{\sigma}(x, \delta)\right)^{\xi(x, \delta)}+G\left(\operatorname{ext} \Phi_{\eta, \theta}\right)(x, \delta),
\end{aligned}
$$

где $G: L^{n}[a, b] \rightarrow C^{n}[a, b]$ - оператор Грина, матрица $X$ определена в п. 3.1 , то из теоремы 9 вытекает следуюшее утверждение.

ТЕОРемА 16. Пусть $U$ - выпуклое компактное множество пространства $C^{n}[a, b]$. Тогда для любих $\eta(\cdot, \cdot) \in K([a, b] \times[0, \infty)), \theta(\cdot, \cdot), \sigma(\cdot, \cdot)$, $\xi(\cdot, \cdot) \in P\left(C^{n}[a, b] \times[0, \infty)\right)$ справедливы равенства

$$
H_{\mathrm{co}}^{1}(U)=\bigcap_{\delta>0} \overline{\operatorname{ext} H_{\eta(\delta), \theta(\delta), \sigma(\delta), \xi(\delta)}^{1}(U)}=\bigcap_{\delta>0} \overline{H_{\eta(\delta), \theta(\delta), \sigma(\delta), \xi(\delta)}^{1}(U)},
$$

где $\overline{\operatorname{ext} H_{\eta(\delta), \theta(\delta), \sigma(\delta), \xi(\delta)}^{1}(U)}, \overline{H_{\eta(\delta), \theta(\delta), \sigma(\delta), \xi(\delta)}^{1}(U)}$ - замыкания в пространстве $C^{n}[a, b]$ множеств решений $\operatorname{ext} H_{\eta(\delta), \theta(\delta), \sigma(\delta), \xi(\delta)}^{1}(U), H_{\eta(\delta), \theta(\delta), \sigma(\delta), \xi(\delta)}^{1}(U)$ соответственно. 


\section{Список литературы}

1. Fryszkowski A. Continuous selection for a class of non-convex multivalued maps // Studia Math. 1983. V. 76. № 2. P. 163-174.

2. Булгаков А.И. К вопросу существования непрерьвных ветвей у многозначных отображений с невьпукльми образами в пространствах суммируемых функций // Матем. сб. 1988. Т. 136 (178). № 2. С. 292-300.

3. Булгаков А.И., Ткач Л. И. Некоторые результаты по теории возмущений многозначных операторов с выпуклыми замкнутьми значениями отображением типа Гаммерштейна с невьпукльпи образами и их приложения // Вестн. ТамбГУ. Сер. естеств. и технич. науки. 1997. Т. 2. № 2. С. 111-120.

4. Булгаков А.И., Ткач Л.И. Асимптотическое представление множеств $\delta$-решений включения типа Гаммерштейна // Вестн. ТамбГУ. Сер. естеств. и технич. науки. 1997. T. 2. № 3. C. 294-298.

5. Булгаков А.И., Ткач Л.И. Возмущение вьпуклозначного оператора многозначньмм отображением типа Гаммерштейна с невьпукльми образами и краевые задачи для функционально-дифференциальных включений // Матем. сб. 1998. Т. 189. №6. С. 3-32.

6. Булгаков А.И., Ткач Л.И. Возмущение однозначного оператора многозначньпм отображением типа Гаммерштейна с невыпуклыми образами // Изв. вузов. Сер. матем. 1999. №3. C. 3-16.

7. Michal E. A. Continuous selection. I // Ann. Math. 1956. V. 63. № 2. P. 361-381.

8. Иоффе А. Д., Тихомиров В. М. Теория экстремальных задач. М.: Наука, 1974.

9. Натансон И. Т. Теория функций вещественной переменной. М.: Наука, Физматлит, 1974.

10. Булгаков А. И. Непрерьвные ветви многозначных отображений и интегральные включения с невыпуклыми образами и их приложения. I // Дифференц. уравнения. 1992. Т. 28. №3. С. 371-379; II // Дифференц. уравнения. 1992. Т. 28. № 4. С. 566-571; III // Дифференц. уравнения. 1992. Т. 28. № 5. С. 739-746.

11. Благодатских В.И., Филиппов А. Ф. Дифференциальные включения и оптимальное управление // Труды МИАН. 1985. Т. 169. С. 194-252.

12. Филиппов $A . \Phi$. Классические решения дифференциальных уравнений с многозначной правой частью // Вестн. МГУ. Сер. 1. Матем., мех. 1967. №3. С. 16-26.

13. Булгаков А. И. Функционально-дифференциальное включение с оператором, имеющим невьптуклые образы // Дифференц. уравнения. 1987. Т. 23. № 10. С. 1659-1668.

14. Филиппов A. Ф. Диффференциальные уравнения с разрывной правой частью. М.: Наука, 1985.

15. Wazewski T. Sur une generalisation de la notion des solutions d'une equation au contingent // Bull. Acad. Polon. Sci. Sér. Sei. Math. Astronom. Phys. 1962. V. 10. № 1. P. 11-15.

16. Булгаков А.И., Скоморохов В. В. Аппроксимация диффференциальных включений // Матем. сб. 2002. Т. 193. № 2. С. 35-52.

17. Булгаков А.И. Интегральные включения с невыпуклыми образами и их приложения к краевым задачам дифференциальных включений // Матем. сб. 1992. Т. 183. № 10. C. $63-86$.

18. Толстоногов A. А. Дифференциальные включения в банаховом пространстве. Новосибирск: Наука, 1986.

19. Булгаков А.И., Ляпин Л. Н. Некоторые свойства множества решений интегрального включения Вольтерра-Гаммерштейна // Дифференц. уравнения. 1978. Т. 14. № 8 . C. $1465-1472$.

20. Plis A. Traejectories and quasitrajectories of an orientor field // Bull. Acad. Polon. Sci. Sér. Math. Astronom. Phys. 1963. V. 11. №6. P. 369-370.

21. Суслов С.И. Нелинейный бэнг-бэнг принцип I. Конечномерный случай // Препринт. № 11. Новосибирск: Ин-т матем. СО АН СССР, 1989.

22. Суслов С.И. Нелинейный бэнг-бэнг принцип II. Бесконечномерный случай // Препринт. № 12. Новосибирск: Ин-т матем. СО АН СССР, 1989. 
23. Толстоногов А. А., Чугунов П.И. О множестве решений дифференциалшного включения в банаховом пространстве // Сиб. матем. журн. 1983. Т. 24. №6. С. 144-159.

24. Толстоногов A.A., Финогенко И.A. О решениях дифференциального включения с полунепрерывной снизу невыпуклой правой частью в банаховом пространстве // Матем. сб. 1984. Т. 125 (167). № 2. С. 199-230.

25. Чугунов П.И. Свойства решений дифференциальных включений и управляемые системы // Прикладная математика и пакеты прикладных программ. Иркутск: Изд-во СЭИСО АН СССР, 1980. С. 155-179.

26. Bressan A. On a bang-bang principle for nonlinear systems // Boll. Un. Mat. Ital. Suppl. 1980. V. 1. P. 53-59.

27. Hermes $H$. On continuous and measurable selections and the existence of solutions of generalized differential equations // Proc. Amer. Math. Soc. 1971. V. 29. № 3. P. 535-542.

28. Pianigiani $G$. On the fundamental theory of multivalued differential equations // J. Differential Equations. 1977. V. 25. № 1. P. 30-38.

29. Булгаков А.И., Григоренко А. А., Жуковский Е. С. Возмущенные включения с компактнозначным отображением // Вестн. УдмГУ. Матем., мех. 2000. № 1. С. 33-40.

30. Ирисов A.E., Тонков E. Л. О замькании множества периодических решений диффференциального включения // Дифференциальные и интегральные уравнения. Горький: Изд-во ГГУ, 1983. С. 32-38.

31. Булгаков А.И., Ефремов А. А., Панасенко Е. А. К вопросу устойчивости дифференциалњных включений // Вестн. ТамбГУ. Сер. естеств. и технич. науки. 1999. Т. 4 . № 4. C. $461-470$.

32. Булгаков А.И., Ефремов А.А., Панасенко Е. А. Обыкновенные дифференциальные включения с внутренними и внешними возмущениями // Дифференц. уравнения. 2000. Т. 36. № 12. С. 1587-1598.

33. Булгаков А.И., Пучков Н. П., Скоморохов В. В. Приближенные решения дифференциалњного включения с невьпуклой правой частью // Вестн. ТамбГУ. Сер. естеств. и технич. науки. 2002. Т. 7. № 1. С. 104-105.

34. Обен Ж.-П., Экланд И. Прикладной нелинейный анализ. М.: Мир, 1988.

35. Hajek O. Discontinuous differential equations. I, II // J. Differential Equations. 1979. V. 32. № 2. P. 149-170, 171-185.

36. Булгаков А.И. Асимптотическое представление множеств $\delta$-решений дифференциального включения // Матем. заметки. 1999. Т. 65. № 5. С. 775-778.

37. Булгаков А.И., Скоморохов В. В. Дифференциалњные включения с внешними возмущениями, радиус которых зависит от фазовой переменной // Вестн. ТамбГУ. Сер. естеств. и технич. науки. 2000. Т. 5. № 4. С. 429-430.

38. Ефремов A. A., Панасенко E. А. Устойчивость периодических и двухточечных краевых задач относительно внешних возмущений // Вестн. ТамбГУ. Сер. естеств. и технич. науки. 2000. Т. 5. № 4. С. 446-447.

39. Азбелев Н.В., Максимов В.П., Рахматуллина Л.Ф. Введение в теорию функционально-диффференциальных уравнений. М.: Наука, 1991.

40. Половинкин E. C. Теория многозначных отображений. М.: МФТИ, 1982.

41. Благодатских В. И. Некоторые результаты по теории дифференциальных включений // Summer School on Ordinary Differential Equations. Czechoslovakia, Brno. Part II. 1974. P. 29-67.

42. Субботин A. И., Ченцов А. Г. Оптимизация гарантии в задачах управления. М.: Наука, 1981.

43. Благодатских В. И. Теория дифференциальных включений. Часть І. М.: Изд-во МГУ, 1979.

44. Борисович Ю. Г., Гельман Б. Д., Мьикис А. Д., Обуховский В. В. Введение в теорию многозначных отображений. Воронеж: Изд-во ВГУ, 1986.

45. Булгаков А. И. Некоторые вопросы дифференциальных и интегральных включений с невьпуклой правой частью // Функционально-дифференциальные уравнения. Пермь: ППИ, 1991. С. 28-57. 
46. Арутюнов А. В. Условия экстремума. Анормалњњые и вырожденные задачи. М.: Факториал, 1997.

47. Hermes $H$. The generalised differential equation $\dot{x}(t) \in R(t, x) / /$ Adv. Math. 1970. V. 4 . № 2. P. 149-169.

48. Булгаков А. И. Непрерьвные ветви многозначных отображений с невьпукльми образами и функционально-дифференциальные включения // Матем. сб. 1990. Т. 181. №11. C. $1427-1442$.

49. Булгаков А.И. Функционально-дифференциальные включения с невыпуклой правой частью // Дифференц. уравнения. 1990. Т. 26. №11. С. 1872-1878.

Тамбовский государственньй университет

им. Г.Р. Державина

Поступила в редакцию

E-mail: aib@tsu.tmb.ru

20.04 .2004 и 22.11 .2004 